\title{
SEMI-HOMOGENEOUS SHEAVES, FOURIER-MUKAI TRANSFORMS AND MODULI OF STABLE SHEAVES ON ABELIAN SURFACES
}

\author{
SHINTAROU YANAGIDA AND KŌTA YOSHIOKA
}

\begin{abstract}
This paper studies stable sheaves on abelian surfaces of Picard number one. Our main tools are semi-homogeneous sheaves and Fourier-Mukai transforms. We introduce the notion of semi-homogeneous presentation and investigate the behavior of stable sheaves under Fourier-Mukai transforms. As a consequence, an affirmative proof is given to the conjecture proposed by Mukai in the 1980s. This paper also includes an explicit description of the birational correspondence between the moduli spaces of stable sheaves and the Hilbert schemes.
\end{abstract}

\section{Contents}

1. Introduction

2. Preliminaries

2.1. Mukai lattice and Mukai vector

2.2. Moduli spaces of stable sheaves

2.3. Fourier-Mukai transforms on abelian surfaces

2.4. Semi-homogeneous sheaves

3. Semi-homogeneous presentation of sheaves

3.1. Definitions and basic properties

3.2. Numerical criterion of semi-homogeneous presentation

4. Moduli of simple complexes of numerical invariant $\left(v_{1}, v_{2}, \ell_{1}, \ell_{2}\right)$

4.1. Complexes associated to numerical solutions

4.2. Existence and some properties of the moduli spaces

4.3. Proof of Theorem 3.6

5. Applications of the semi-homogeneous presentation

5.1. An old conjecture of Mukai

5.2. Sheaf-preserving Fourier-Mukai transforms

6. Matrix description of cohomological FMT

6.1. Cohomological correspondence

6.2. Some calculations for matrix description

6.3. Matrix description

7. Tame complex

7.1. The arithmetic group $G$ and numerical solutions for the ideal sheaf

7.2. Construction of the tame complex: case I

7.3. Application of the tame complex.

7.4. Construction and the application of the tame complex: case II

8. Birational morphism of the moduli

8.1. Quadratic forms and the description of the birational map

8.2. Structures of $G$

Date: June 25, 2009.

2000 Mathematics Subject Classification. 14D20.

Key words and phrases. moduli of stable sheaves, semi-homogeneous sheaf, Fourier-Mukai transform.

The first author is supported by JSPS Research Fellowships for Young Scientists (No. 21.2241).

The second author is supported by the Grant-in-aid for Scientific Research (No. 18340010), JSPS. 


\section{INTRODUCTION}

This paper analyzes stable sheaves on abelian surfaces using two notions. The first is the semi-homogeneous sheaves and the second is the Fourier-Mukai transforms. Both of them were introduced by Mukai [M1, M4].

Semi-homogeneous sheaves are well-behaved coherent sheaves on abelian surfaces. They are semi-stable for any ample divisor, and their cohomological properties and classifications were fully investigated in M1. Semi-homogeneous sheaves may be regarded as building blocks of more complicated stable sheaves.

We consider presenting stable sheaves as kernel or cokernel of morphisms between semihomogeneous sheaves. These presentations will be called semi-homogeneous presentations.

Definition (Definition 3.1). For $E \in \operatorname{Coh}(X)$, a semi-homogeneous presentation is an exact sequence

$$
0 \rightarrow E \rightarrow E_{1} \rightarrow E_{2} \rightarrow 0 \quad \text { or } \quad 0 \rightarrow E_{1} \rightarrow E_{2} \rightarrow E \rightarrow 0
$$

with the condition $v\left(E_{1}\right)=\ell_{1} v_{1}, v\left(E_{2}\right)=\ell_{2} v_{2}$ and

$$
\begin{aligned}
& \ell_{1}, \ell_{2} \in \mathbb{Z}_{>0}, \quad\left(\ell_{1}-1\right)\left(\ell_{2}-1\right)=0, \\
& \left\langle v_{1}, v_{2}\right\rangle=-1, \quad v_{1}, v_{2}: \text { positive, primitive and isotropic Mukai vectors. }
\end{aligned}
$$

The uniqueness of the presentation and the conditions for its existence will be clarified in $\S 3$ under the condition that the Picard number of the surface is one. In the main Theorem 3.6, we show that a numerical condition on Chern characteristics (or Mukai vectors) of stable sheaves totally controls the existence of the semi-homogeneous presentations. The condition is described by an indefinite equation, called numerical equation (see Definition 3.11). This equation plays an important role in this paper.

The proof of Theorem 3.6 requires moduli space $\mathfrak{M}^{+}\left(v_{1}, v_{2}, \ell_{1}, \ell_{2}\right)$ of simple two-term complexes associated to the solution $\left(v_{1}, v_{2}, \ell_{1}, \ell_{2}\right)$ of the numerical equation. We construct these spaces in $\S 4$. These spaces are essentially the Hilbert schemes of points over the abelian surfaces and enable us to treat the family of two-term complexes including the desired semi-homogeneous presentations.

As an application of the semi-homogeneous presentation, we prove the Conjecture 5.1, originally proposed by Mukai [M3]:

Conjecture (Conjecture 5.1). Let $X$ be an abelian surface with $\operatorname{NS}(X)=\mathbb{Z} H$ and $v$ be a Mukai vector with $\ell:=\left\langle v^{2}\right\rangle / 2>0$. Suppose that $v$ has at least one solution of the numerical equation (3.2).

(1) Among the numerical solutions for $v$, take the solution $\left(v_{1}, v_{2}, \ell_{1}, \ell_{2}\right)$ such that $\operatorname{rk}\left(v_{i}\right)$ is minimum, where the index $i \in\{1,2\}$ is determined by $\ell_{i}=\ell$. Then for a general member $\left[E^{-1} \stackrel{f}{\rightarrow} E^{0}\right]$ of $\mathfrak{M}^{+}\left(v_{1}, v_{2}, \ell_{1}, \ell_{2}\right), f$ is either surjective or injective.

(2) In the situation of (1), the kernel or cokernel of $f$ is stable.

(3) A general member of $M_{X}^{H}(v)$ has a semi-homogeneous presentation corresponding to the numerical solution $\left(v_{1}, v_{2}, \ell_{1}, \ell_{2}\right)$ of $v$ such that $\operatorname{rk}\left(v_{i}\right)$ is minimum, where the index $i \in\{1,2\}$ is determined by $\ell_{i}=\ell$.

Theorem (Theorem 5.2). The above conjecture is true. 
We also obtained some explicit conditions when sheaves are transformed into sheaves under Fourier-Mukai transforms. These will be explained in $\S 5$.

In order to see how semi-homogeneous presentations behave under a Fourier-Mukai transform, we need to calculate Mukai vectors explicitly. One can describe Mukai vectors economically using quadratic matrices, which will be explained in $\S 6$. These matrices turn out to form an arithmetic group $G$, introduced in Definition 6.9. During the study of this matrix description, we are led to the study of cohomological representation of the equivalences among the derived categories of coherent sheaves on abelian surfaces. The result is shown in Theorem 6.16.

In the subsequent $\S 7$, we extensively use the semi-homogeneous presentation and the matrix description to analyze the behavior of stable sheaves under Fourier-Mukai transforms. The resulting image is in general a complex, and the analysis is too complicated. We circumvent this difficulty by constructing a sequence of tame complexes, for which one can completely describe the cohomology sheaves and the behavior under Fourier-Mukai transforms. As a consequence we obtained Theorems 7.21 and 7.31, which give the numerical condition under which a stable sheaf is transformed into a sheaf by Fourier-Mukai transforms.

In $\S 8$, we present an explicit construction of the birational correspondence between the moduli spaces of stable sheaves and the Hilbert schemes. The main result is shown in Proposition 8.2. The birational correspondence requires the existence of a solution of the numerical solution and the resulting birational map is realized by a Fourier-Mukai transform. In the final $\S \S 8.3$, we treat the case of the principally polarized abelian surfaces. In this case the arithmetic group $G$ introduced in $\S[$ reduces to $\mathrm{SL}(2, \mathbb{Z})$, and the condition above reduces to the existence of solutions of integral quadratic forms. Thus using the classical theory of quadratic forms we can obtain a clear description of the birational maps between moduli and the Hilbert schemes, as shown in

Theorem (Theorem 8.10). Let $X$ be a principally polarized abelian surface with $\operatorname{NS}(X)=\mathbb{Z} H$. Let $v=(r, d H, a)$ be a Mukai vector satisfying the following condition.

$$
\ell:=\left\langle v^{2}\right\rangle / 2 \in \mathbb{Z}_{>0} \text { is not a square number, and }
$$

the class number of quadratic forms with discriminant $\ell$ is 1 .

Then the birational morphism $M_{X}^{H}(v) \cdots \rightarrow X \times \operatorname{Hilb}^{\ell} X$ is given by the following description.

(1) Take a Mukai vector $v_{1}=\left(p_{1}^{2}, p_{1} q_{1} H, q_{1}^{2}\right)$ which is of minimum rank among those satisfying $2 p_{1} q_{1} d-p_{1}^{2} a-q_{1}^{2} r=\epsilon$, where $\epsilon=1$ or -1 . Then

$$
\gamma:= \pm\left[\begin{array}{cc}
q_{1} & \epsilon\left(d q_{1}-a p_{1}\right) \\
-p_{1} & \epsilon\left(d p_{1}-r q_{1}\right)
\end{array}\right] \in \operatorname{PSL}(2, \mathbb{Z})
$$

diagonalizes the matrix $Q_{v}=\left[\begin{array}{ll}r & d \\ d & a\end{array}\right]$, that is, ${ }^{t} \gamma Q_{v} \gamma=-\epsilon\left[\begin{array}{cc}1 & 0 \\ 0 & -\ell\end{array}\right]$.

(2) Set $v_{2}:=\left(p_{2}^{2}, p_{2} q_{2} H, q_{2}^{2}\right)$, where $q_{2}=-\epsilon\left(d q_{1}-a p_{1}\right)$ and $p_{2}=\epsilon\left(d p_{1}-r q_{1}\right)$. Then a general member of $M_{X}^{H}(v)$ has a semi-homogeneous presentation. Moreover the FMT $\Phi:=\Phi_{X \rightarrow X}^{\mathbf{E}}$ such that $\theta(\Phi)=\gamma$ or the composition $\mathcal{D}_{X} \circ \Phi$ gives the birational correspondence $M_{X}^{H}(v) \cdots \rightarrow X \times \operatorname{Hilb}^{\ell}(X)$ up to shift.

Acknowledgements. In the final stage for preparing this article, the second named author stayed at Max Planck Institut für Mathematik. He would like to thank the institution for the hospitality and support very much.

Notation. All schemes are of finite type over $\mathbb{C}$. For coherent sheaves $E$ and $F$ on a scheme $X$, we abbreviate $\operatorname{Hom}_{\mathcal{O}_{X}}(E, F)$ and $\operatorname{Ext}_{\mathcal{O}_{X}}^{i}(E, F)$ to $\operatorname{Hom}(E, F)$ and $\operatorname{Ext}^{i}(E, F)$.

The abelian category of coherent sheaves on a projective variety $X$ is denoted by $\operatorname{Coh}(X)$, and the bounded derived category of $\operatorname{Coh}(X)$ is denoted by $\mathbf{D}(X)$. An object of $\mathbf{D}(X)$, namely a 
complex of coherent sheaves on $X$, will be denoted like $E^{\bullet}$. We also use the symbol $\left[E^{-1} \rightarrow E^{0}\right]$ for a two-term complex. In this notation, the $(-1)$-th and the zeroth component of the complex are $E^{-1}$ and $E^{0}$, and the other components are the zero sheaf. For an object $E^{\bullet} \in \mathbf{D}(X), H^{i}\left(E^{\bullet}\right)$ means the $i$-th cohomology sheaf of $E^{\bullet}$. We denote $\operatorname{Hom}_{\mathbf{D}(X)}\left(E^{\bullet}, F^{\bullet}\right)$ and $\operatorname{Ext}_{\mathbf{D}(X)}^{i}\left(E^{\bullet}, F^{\bullet}\right)$ by $\operatorname{Hom}\left(E^{\bullet}, F^{\bullet}\right)$ and $\operatorname{Ext}^{i}\left(E^{\bullet}, F^{\bullet}\right)$ if confusion does not arise. We also denote the opposite category of $\mathbf{D}(X)$ by $\mathbf{D}(X)_{\text {op }}$ and the dualizing functor by

$$
\mathcal{D}_{X}: \mathbf{D}(X) \rightarrow \mathbf{D}(X)_{\mathrm{op}}, \quad x \mapsto x^{\vee}:=\mathbf{R} \mathcal{H} \text { om }\left(x, \mathcal{O}_{X}\right) .
$$

Two smooth projective varieties $X$ and $Y$ are said to be Fourier-Mukai partners if there is an equivalence $\mathbf{D}(X) \simeq \mathbf{D}(Y)$. The set of isomorphism classes of Fourier-Mukai partners of $X$ is denoted by $\operatorname{FM}(X)$. The set of equivalences between $\mathbf{D}(X)$ and $\mathbf{D}(Y)$ is denoted by $\operatorname{Eq}(\mathbf{D}(X), \mathbf{D}(Y))$.

For an abelian variety $X$, its dual variety $\operatorname{Pic}^{0}(X)$ is denoted as $\widehat{X}$. The Poincaré line bundle of $X$ is denoted by $\mathbf{P}_{X}$ and we sometimes abbreviate it to $\mathbf{P}$ if confusion does not occur.

Assume that an ample divisor $H$ is fixed on a projective variety $X$. For $E \in \operatorname{Coh}(X)$, we define its slope by $\mu(E):=\left(c_{1}(E), H\right) / \operatorname{rk}(E)$ if $\operatorname{rk}(E)>0$. Here $(\cdot, \cdot)$ is the intersection form on $H^{2}(X, \mathbb{Q})$. If $\operatorname{rk}(E)=0$, then we define $\mu(E):=+\infty$.

In this paper we use the word 'stability' in the sense of Simpson (for detail, see [HL]). $M_{X}^{H}(v)^{s s}$ denotes the coarse moduli scheme which parametrizes $S$-equivalent classes of semi-stable shaves $E$ on $X$ having $v$ as their Mukai vectors (see $\S \S 2.1$ for the definition). We denote by $M_{X}^{H}(v)$ the open subscheme consisting of stable sheaves.

\section{Preliminaries}

2.1. Mukai lattice and Mukai vector. First we introduce the Mukai lattice [M6] for an abelian surface $X$. Consider the lattice $\left(H^{\mathrm{ev}}(X, \mathbb{Z}),\langle\cdot, \cdot\rangle\right)$ consisting of the even part of the cohomology group

$$
H^{\mathrm{ev}}(X, \mathbb{Z}):=\bigoplus_{i=0}^{2} H^{2 i}(X, \mathbb{Z}),
$$

and the Mukai pairing $\langle\cdot, \cdot\rangle$ defined as

$$
\begin{aligned}
\langle x, y\rangle:= & \int_{X}\left(x_{1} \cup y_{1}-x_{0} \cup y_{2}-x_{2} \cup y_{0}\right), \\
& x=\left(x_{0}, x_{1}, x_{2}\right), y=\left(y_{0}, y_{1}, y_{2}\right), x_{i}, y_{i} \in H^{2 i}(X, \mathbb{Z}) .
\end{aligned}
$$

This paring is symmetric and bilinear. We also have $\left(H^{\mathrm{ev}}(X, \mathbb{Z}),\langle\cdot, \cdot\rangle\right) \cong U^{\perp 4}$, where $U$ is the hyperbolic lattice and $\perp$ is the orthogonal direct sum. In particular, $\left(H^{\mathrm{ev}}(X, \mathbb{Z}),\langle\cdot, \cdot\rangle\right)$ is a non-degenerate even lattice.

For an object $E^{\bullet} \in \mathbf{D}(X)$, the Mukai vector of $E^{\bullet}$ is defined as its Chern character.

$$
v\left(E^{\bullet}\right):=\operatorname{ch}\left(E^{\bullet}\right)=\left(\operatorname{rk}\left(E^{\bullet}\right), c_{1}\left(E^{\bullet}\right), \chi\left(E^{\bullet}\right)\right) \in H^{\mathrm{ev}}(X, \mathbb{Z}) .
$$

By the Grothendieck-Riemann-Roch formula we have

$$
\left\langle v\left(E^{\bullet}\right), v\left(F^{\bullet}\right)\right\rangle=-\chi\left(E^{\bullet}, F^{\bullet}\right)
$$

for $E^{\bullet}, F^{\bullet} \in \mathbf{D}(X)$. For a class $e \in K(X)$ of the Grothendieck group of $X$, we similarly define $v(e):=\operatorname{ch}(e)=\left(\operatorname{rk}(e), c_{1}(e), \chi(e)\right)$.

We call an element $v \in H^{\mathrm{ev}}(X, \mathbb{Z})$ a Mukai vector if there exists an object $E^{\bullet} \in \mathbf{D}(X)$ such that $v=v\left(E^{\bullet}\right)$. The set of Mukai vectors is nothing but the submodule $H^{\mathrm{ev}}(X, \mathbb{Z})_{\text {alg }}$ of $H^{\mathrm{ev}}(X, \mathbb{Z})$ defined by

$$
H^{\mathrm{ev}}(X, \mathbb{Z})_{\mathrm{alg}}:=H^{0}(X, \mathbb{Z}) \oplus \mathrm{NS}(X) \oplus H^{4}(X, \mathbb{Z}) .
$$


Here $\operatorname{NS}(X)$ denotes the Néron-Severi group of $X .\left(H^{\mathrm{ev}}(X, \mathbb{Z})_{\text {alg }},\langle\cdot, \cdot\rangle\right)$ is called the Mukai lattice of $X$.

We list several definitions concerning Mukai vectors.

Definition 2.1. Let $v=(r, \xi, a)$ be a Mukai vector. $v$ is called isotropic if $\left\langle v^{2}\right\rangle:=\langle v, v\rangle=0$. $v$ is called positive and denoted by $v>0$ if $r>0$, or if $r=0$ and $\xi$ is effective, or if $r=0$ and $\xi=0$ and $a>0 . v$ is called primitive if $H^{\mathrm{ev}}(X, \mathbb{Z}) /(\mathbb{Z} v)$ is torsion free. For a non-zero Mukai vector $v$, its multiplicity $\ell$ is defined to be the non-negative integer defined by the relation $v=\ell w$, where $w$ is a primitive Mukai vector. We also define $\operatorname{rk}(v):=r, c_{1}(v):=\xi$ and $\chi(v):=a$. When an ample divisor $H$ is fixed, we define $\mu(v):=(\xi, H) / r$ if $r>0$ and $\mu(v):=+\infty$ if $r=0$. At last, we define $v^{\vee}:=(r,-\xi, a)$.

In this article we sometimes denote a Mukai vector $v=(r, \xi, a)$ as $v=r+\xi+a \rho_{X}$ additively. Here $\rho_{X}$ is the element of $H^{4}(X, \mathbb{Z})$ satisfying $\int_{X} \rho_{X}=1$. Also we will use the exponential form of the Chern characteristics. For example, for $d \in \mathbb{Z}$ and an ample divisor $H, e^{d H}$ means $\left(1, d H, d^{2}\left(H^{2}\right) / 2\right)$.

2.2. Moduli spaces of stable sheaves. We mention only a few properties of the moduli of stable sheaves, although there are considerable amount of study.

Definition 2.2. Let $v$ be a Mukai vector. An ample divisor $H$ is general with respect to $v$, if the following condition holds. For any $\mu$-semi-stable sheaf $E$ with $v(E)=v$, if $F \subset E$ satisfies $\left(c_{1}(F), H\right) / \operatorname{rk}(F)=\left(c_{1}(E), H\right) / \operatorname{rk}(E)$, then $c_{1}(F) / \operatorname{rk}(F)=c_{1}(E) / \operatorname{rk}(E)$.

Fact 2.3. Let $v=(r, \xi, a)$ be a Mukai vector of $r>0$ and $\left\langle v^{2}\right\rangle>0$, and $H$ be an ample divisor which is general with respect to $v$. Then $M_{X}^{H}(v)$ is smooth, projective and irreducible.

Proof. Smoothness and projectivity are proved in [M5]. Irreducibility is proved in [Y2, Theorem $3.18]$.

2.3. Fourier-Mukai transforms on abelian surfaces. The next ingredient is the FourierMukai transform initiated by Mukai in [M4]. In this subsection we summarize its main properties, focusing on the case of abelian surfaces. For a general treatment of this functor we recommend the reader to see $[\mathrm{Hu}]$.

Definition 2.4. Let $X$ and $Y$ be smooth projective varieties. For an object $\mathbf{E}^{\bullet} \in \mathbf{D}(X \times Y)$, the integral functor $\Phi_{X \rightarrow Y}^{\mathbf{E}^{\bullet}}$ is defined as

$$
\begin{aligned}
& \Phi_{X \rightarrow Y}^{\mathbf{E}^{\bullet}}: \quad \mathbf{D}(X) \rightarrow \quad \mathbf{D}(Y) \\
& x \mapsto \mathbf{R} p_{Y *}\left(\mathbf{E}^{\bullet} \stackrel{\mathbf{L}}{\otimes} p_{X}^{*}(x)\right),
\end{aligned}
$$

where $p_{X}: X \times Y \rightarrow X$ and $p_{Y}: X \times Y \rightarrow Y$ are the natural projections. We call $\mathbf{E}^{\bullet}$ its kernel. If $\Phi_{X \rightarrow Y}^{\mathbf{E}^{\bullet}}$ is an exact equivalence, it is called a Fourier-Mukai transform. In this paper we abbreviate it to FMT.

In order to describe FMT in detail, it is convenient to introduce the next terminology.

Definition 2.5. Let $X$ and $Y$ be smooth projective varieties, $E$ be a coherent sheaf over $X$ and $\Phi: \mathbf{D}(X) \rightarrow \mathbf{D}(Y)$ be an equivalence. We say that $E$ satisfies the weak index theorem with respect to $\Phi$ and that its index is equal to $i$ if $H^{j}(\Phi(E))=0$ for every $j \neq i$. If this condition holds, then we also say that $\mathrm{WIT}_{i}$ holds for $E$ with respect to $\Phi$.

We will extensively utilize the following classification of FMTs on abelian surfaces due to Orlov $\mathrm{Or}$. For the proof, see [Y4, Theorem 1.4].

Fact 2.6. (1) Let $X$ be an abelian surface and $Y$ be a smooth projective variety. If $\Phi_{X \rightarrow Y}^{\mathrm{E}^{\bullet}}$ is a FMT, then $Y$ is an abelian surface and the inverse functor is given by $\Phi_{Y \rightarrow X}^{\mathbf{E}^{\bullet \vee}[2]}$. 
(2) Let $X$ be an abelian surface and $v=(r, \xi, a)$ be a primitive isotropic Mukai vector. Suppose that there exists a universal family $\mathbf{E}$ on $M_{X}^{H}(v) \times X$. Then $\Phi_{X \rightarrow M_{X}^{H}(v)}^{\mathbf{E}}$ is a FMT.

(3) Let $X$ and $Y$ be abelian surfaces. Suppose that there exists an equivalence $\Phi: \mathbf{D}(X) \rightarrow$ $\mathbf{D}(Y)$. Then there exists a primitive isotropic Mukai vector $v$ such that $Y=M_{X}^{H}(v)$. Moreover there exists a universal family $\mathbf{E}$ on $Y \times X$ and an integer $k \in \mathbb{Z}$ such that $\Phi=\Phi_{X \rightarrow Y}^{\mathbf{E}[k]}$.

Let $\Phi=\Phi_{X \rightarrow Y}^{\mathrm{E}^{\bullet}}$ be a FMT between abelian surfaces $X$ and $Y$. This functor induces an isomorphism $\Phi^{K}: K(X) \rightarrow K(Y)$ on the Grothendieck groups and an isomorphism $\Phi^{H}: H^{\mathrm{ev}}(X, \mathbb{Q}) \rightarrow$ $H^{\mathrm{ev}}(Y, \mathbb{Q})$ on the cohomology groups. Moreover, by the Grothendieck-Riemann-Roch formula, the following diagram is commutative. (For the proof, see [Hu, $\S \S 5.2]$.)

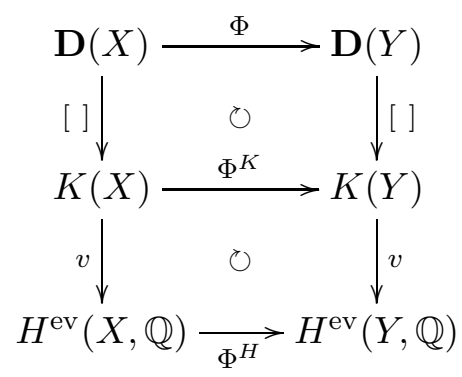

Here the symbol [] means taking the class of the object and $v$ means taking the Mukai vector.

Moreover, one can prove that $\Phi^{H}(x) \in H^{\mathrm{ev}}(Y, \mathbb{Z})$ for $x \in H^{\mathrm{ev}}(X, \mathbb{Z})$ and that $\Phi^{H}$ induces isometries $\left(H^{\mathrm{ev}}(X, \mathbb{Z}),\langle\cdot, \cdot\rangle\right) \rightarrow\left(H^{\mathrm{ev}}(Y, \mathbb{Z}),\langle\cdot, \cdot\rangle\right)$ and $\left(H^{\mathrm{ev}}(X, \mathbb{Z})_{\mathrm{alg}},\langle\cdot, \cdot\rangle\right) \rightarrow\left(H^{\mathrm{ev}}(Y, \mathbb{Z})_{\mathrm{alg}},\langle\cdot, \cdot\rangle\right)$. (For the proof, see [Y1, Lemma 2.2].) $\Phi^{H}$ will be called the cohomological FMT induced by $\Phi$. We often suppress the symbol $H$ of the cohomological FMT and simply denote it by $\Phi$, using the same notation of the original FMT.

Lemma 2.7. Let $X$ be an abelian surface with $\mathrm{NS}(X)=\mathbb{Z} H,\left(H^{2}\right)=2 n$ and let us take $Y \in \operatorname{FM}(X)$. Then $\operatorname{NS}(Y)$ is of rank one. Moreover if we put $\operatorname{NS}(Y)=\mathbb{Z} \widehat{H}$, where $\widehat{H}$ is the ample generator of $\mathrm{NS}(Y)$, then we have $\left(\widehat{H}^{2}\right)=2 n$.

Proof. A FMT $\mathbf{D}(X) \stackrel{\sim}{\rightarrow} \mathbf{D}(Y)$ induces an isometry $H^{\mathrm{ev}}(X, \mathbb{Z})_{\text {alg }} \stackrel{\sim}{\rightarrow} H^{\mathrm{ev}}(Y, \mathbb{Z})_{\text {alg. }}$. Since in our case $H^{\mathrm{ev}}(X, \mathbb{Z})_{\text {alg }} \cong \mathrm{NS}(X) \perp U$ and $H^{\mathrm{ev}}(Y, \mathbb{Z})_{\text {alg }} \cong \mathrm{NS}(Y) \perp U$, where $U$ is the hyperbolic lattice, we find that $\rho(X)+2=\operatorname{rk}\left(H^{\mathrm{ev}}(X, \mathbb{Z})_{\mathrm{alg}}\right)=\operatorname{rk}\left(H^{\mathrm{ev}}(Y, \mathbb{Z})\right.$ alg $)=\rho(Y)+2$ and $\left(H^{2}\right)=$ $-\operatorname{det} H^{\mathrm{ev}}(X, \mathbb{Z})_{\mathrm{alg}}=-\operatorname{det} H^{\mathrm{ev}}(Y, \mathbb{Z})_{\mathrm{alg}}=\left(\widehat{H}^{2}\right)$. Therefore the conclusion holds.

Finally, the Serre duality yields the following lemma.

Lemma 2.8. For a FMT $\Phi_{X \rightarrow Y}^{\mathrm{E}^{\bullet}}: \mathbf{D}(X) \rightarrow \mathbf{D}(Y)$ between abelian surfaces $X$ and $Y$, we have

$$
\mathcal{D}_{Y} \Phi_{X \rightarrow Y}^{\mathbf{E}^{\bullet} \vee}=\Phi_{X \rightarrow Y}^{\mathbf{E}^{\bullet}[2]} \mathcal{D}_{X}
$$

2.4. Semi-homogeneous sheaves. Here we recall the notion of semi-homogeneous sheaves over abelian surfaces, which was introduced by Mukai [M1]. Fix an abelian surface $X$.

Definition 2.9. For any coherent sheaf $E$ on $X$, the subset

$$
S(X):=\left\{(x, \hat{x}) \in X \times \widehat{X} \mid T_{x}^{*}(E) \otimes \mathbf{P}_{\hat{x}} \cong E\right\}
$$

is an abelian subvariety of $X \times \widehat{X}$ with $\operatorname{dim} S(X) \leq 2$ (see [M1, Proposition 3.3]). If $\operatorname{dim} S(X)=$ 2, then $E$ is called semi-homogeneous.

Semi-homogeneous sheaves are classified as below. For the proof, see [M1] and [Y4, §4]. 
Fact 2.10. Let $H$ be an ample divisor on $X$ and $v=(r, \xi, a)$ be a positive isotropic Mukai vector.

(1) Let $E$ be a coherent sheaf with $v(E)=v$. Fix an ample divisor $H$ on $X$.

(a) $E$ is simple $\Longleftrightarrow E$ is stable $\Longleftrightarrow v$ is primitive. The stability of $E$ is independent of $H$.

(b) If $v$ is primitive and $E$ is semi-stable with respect to $H$ and $v(E)=n v$, then $E$ is $S$-equivalent to $\bigoplus_{i=1}^{n} E_{i}, E_{i} \in M_{X}^{H}(v)$. The semi-stability of $E$ is independent of $H$.

(c) Every semi-stable sheaf with isotropic Mukai vector is semi-homogeneous.

(2) Stable semi-homogeneous sheaves are classified as follows.

(a) Semi-homogeneous vector bundle.

For any $\delta \in \mathrm{NS}(X) \otimes_{\mathbb{Z}} \mathbb{Q}$, there is a simple semi-homogeneous vector bundle $E$ satisfying $c_{1}(E) / \mathrm{rk}(E)=\delta$.

If $E$ and $E^{\prime}$ are simple semi-homogeneous vector bundles with $c_{1}(E) / \operatorname{rk}(E)=$ $c_{1}\left(E^{\prime}\right) / \operatorname{rk}\left(E^{\prime}\right)$, then $E \cong E^{\prime} \otimes M$ for some $M \in \operatorname{Pic}^{0}(X)$.

For any semi-homogeneous vector bundle $E$, there exist an isogeny $\pi: X^{\prime} \rightarrow X$ of abelian surfaces and a line bundle $L$ on $X^{\prime}$ such that $\pi_{*}(L) \cong E$.

(b) Torsion semi-homogeneous sheaves.

$\operatorname{Supp}(E)$ is an elliptic curve $C$ and $E$ is a locally free semi-stable sheaf over $C$, or $\operatorname{Supp}(E)$ is one point and $E$ is a skyscraper sheaf.

Remark 2.11. Notice that if $\operatorname{NS}(X)=\mathbb{Z} H$ then the first case in (2) (b) does not occur. Therefore non-locally-free semi-homogeneous sheaves are nothing but coherent sheaves with finite support.

The cohomology groups of semi-homogeneous sheaves were also investigated by Mukai [M1]. The results are as follows. For the proof, see Mukai's paper or [Y4, Proposition 4.4].

Fact 2.12. Let $E$ and $F$ be semi-homogeneous sheaves.

(1) Suppose that $\operatorname{rk}(E)>0$ and $\operatorname{rk}(F)>0$.

(a) If $\langle v(E), v(F)\rangle>0$, then $\operatorname{Hom}(E, F)=\operatorname{Ext}^{2}(E, F)=0$.

(b) If $\langle v(E), v(F)\rangle<0$, then $\mu(E) \neq \mu(F)$, $\operatorname{Ext}^{1}(E, F)=0$, and

$$
\left\{\begin{array}{l}
\operatorname{Hom}(E, F)=0 \quad \text { if } \quad \mu(E)>\mu(F), \\
\operatorname{Ext}^{2}(E, F)=0 \quad \text { if } \quad \mu(E)<\mu(F),
\end{array}\right.
$$

(2) Suppose that $\operatorname{rk}(E)>0$ and $F$ is a torsion sheaf.

(a) If $\langle v(E), v(F)\rangle>0$, then $\operatorname{Hom}(E, F)=\operatorname{Ext}^{2}(E, F)=0$.

(b) If $\langle v(E), v(F)\rangle<0$, then $\operatorname{Ext}^{1}(E, F)=\operatorname{Ext}^{2}(E, F)=0$.

(3) Suppose $E$ and $F$ are torsion sheaves. Then we have $\langle v(E), v(F)\rangle \geq 0$. If $\langle v(E), v(F)\rangle>$ 0 , then $\operatorname{Hom}(E, F)=\operatorname{Ext}^{2}(E, F)=0$.

Lemma 2.13. Let $E$ and $F$ are semi-homogeneous sheaves on $X$. Assume that $\operatorname{NS}(X)=\mathbb{Z} H$. Then $\langle v(E), v(F)\rangle \leq 0$. If the equality holds, then $\mathbb{Q} v(E)=\mathbb{Q} v(F)$.

Proof. If $\operatorname{rk}(E)>0$ and $\operatorname{rk}(F)>0$, then one can put

$$
v(E)=r_{1} e^{\left(d_{1} / r_{1}\right) H}=\left(r_{1}, d_{1} H, d_{1}^{2}\left(H^{2}\right) /\left(2 r_{1}\right)\right), \quad v(F)=r_{2} e^{\left(d_{2} / r_{2}\right) H}=\left(r_{2}, d_{2} H, d_{2}^{2}\left(H^{2}\right) /\left(2 r_{2}\right)\right),
$$

with $r_{1}, r_{2} \in \mathbb{Z}_{>0}$ and $d_{1}, d_{2} \in \mathbb{Z}$. The Mukai paring becomes $\langle v(E), v(F)\rangle=-\left(r_{1} d_{2}-\right.$ $\left.r_{2} d_{1}\right)^{2}\left(H^{2}\right) /\left(2 r_{1} r_{2}\right) \leq 0$, which yields the first statement. The equality holds if and only if $d_{1} / r_{1}=d_{2} / r_{2}$. This is equivalent to $\mathbb{Q} v(E)=\mathbb{Q} v(F)$ by (2.2). Thus we have the second statement. 
Assume that $\operatorname{rk}(E)=0$. By Remark 2.11 one can put $v(E)=(0,0, a)$ with $a \in \mathbb{Z}_{>0}$. Then $\langle v(E), v(F)\rangle=-a \operatorname{rk}(F) \leq 0$. The equality holds if and only if $\operatorname{rk}(F)=0$ if and only if $v(F)=(0,0, b)$ for some $b \in \mathbb{Z}_{>0}$. Therefore we have the conclusion.

The case $\operatorname{rk}(F)=0$ is the same as the case $\operatorname{rk}(E)=0$.

Proposition 2.14. Assume that $\operatorname{NS}(X)=\mathbb{Z} H$. Let $v$ be a positive, primitive and isotropic Mukai vector and let $Y:=M_{X}^{H}(v)$ be the moduli space. Suppose that there exists a Mukai vector $w$ such that $\langle v, w\rangle=-1$. Then, there exists a universal family $\mathbf{E}$ on $Y \times X$ by [M6, Proposition A.6]. Now set $\Phi:=\Phi_{X \rightarrow Y}^{\mathbf{E}^{\vee}}$ and $\mu:=\mu(v)$. Under these assumptions, the next statements hold.

(1) For any semi-homogeneous sheaf $E$ on $X, \mathrm{WIT}_{i}$ holds w.r.t. $\Phi$ and the index $i$ is

$$
i= \begin{cases}0 & \mu(E)>\mu, \\ 2 & \mu(E) \leq \mu .\end{cases}
$$

Moreover the image $\Phi^{i}(E)$ is a semi-homogeneous sheaf.

(2) For a semi-homogeneous sheaf $E$ with $v(E)=v$, we have $\Phi(E)=\mathbb{C}_{y}[-2]$. Here $y \in Y$ is a closed point corresponding to $E$.

(3) Suppose that the Mukai vector $w$ in the assumption is isotropic. Then for a semihomogeneous sheaf $F$ with $v(F)=w$, we have $\Phi(F)=L[-i]$, where $L$ is a line bundle on $Y$ and the index $i$ is 0 or 2 according to $\mu(F)>\mu$ or $\mu(F) \leq \mu$.

(4) Under the additional condition $\left\langle w^{2}\right\rangle=0$, let $E$ and $F$ be coherent sheaves with $v(E)=v$ and $v(F)=w$ as in (2) and (3). Assume further that $F$ is stable. Then there is a coherent sheaf $\mathbf{F} \in \operatorname{Coh}(X \times Y)$ such that $\Phi^{\prime}:=\Phi_{X \rightarrow Y}^{\mathbf{F}^{\vee}}$ satisfies $\Phi^{\prime}(E)=\mathbb{C}_{y}[-2]$ and $\Phi^{\prime}(F)=\mathcal{O}_{Y}[-i]$.

Proof. (1) Since $\Phi(E)=\mathbf{R} \operatorname{Hom}_{p_{Y}}\left(\mathbf{E}, p_{X}^{*}(E)\right)$, we have $H^{i}(\Phi(E))=\operatorname{Ext}_{p_{Y}}^{i}\left(\mathbf{E}, p_{X}^{*}(E)\right)$. Then from the base change theorem for the relative extension and the cohomological result in Fact 2.12, WIT holds for $E$ and the index coincides with the one given in the statement. Thus the first statement is verified.

Next, we recall that $\Phi$ induces an isometry of the Mukai lattices. Hence $\left\langle v\left(\Phi^{i}(E)\right)^{2}\right\rangle=\left\langle v^{2}\right\rangle=$ 0 . If $E$ is simple, then from this equality and Fact 2.10 (1) (a) we find that $\Phi^{i}(E)$ is a stable semi-homogeneous sheaf. For a proper semi-stable semi-homogeneous sheaf $E, \Phi^{i}(E)$ is a also semi-homogeneous sheaf, since it is an extension of the stable semi-homogeneous sheaves with the same Mukai vectors.

(2) It is an immediate consequence of (1).

(3) From the second statement of (1), we know that $\Phi^{i}(F)$ is a semi-homogeneous sheaf. Its rank is calculated as follows.

$$
\operatorname{rk}\left(\Phi^{i}(F)\right)=\chi\left(\Phi^{i}(F), \mathbb{C}_{y}\right)=\chi\left(F, \Psi^{2-i}\left(\mathbb{C}_{y}\right)\right)=\chi\left(F,\left.\mathbf{E}\right|_{\{y\} \times X}\right)=-\langle w, v\rangle=1 .
$$

Here we used the notation $\Psi:=\Phi_{Y \rightarrow X}^{\mathrm{E}}$ and the fact that $\Psi[2]$ is the inverse of $\Phi$. Thus $\Phi^{i}(F)$ is a semi-homogeneous sheaf of rank 1 , that is, a line bundle.

(4) We use the same notation as in the proof of (3). If $L \cong \mathcal{O}_{Y}$, then it is enough to set $\mathbf{F}:=\mathbf{E}$. Otherwise, by setting $\mathbf{F}:=\mathbf{E} \otimes_{\mathcal{O}_{Y \times X}} p_{Y}^{*} L$, we have the desired conclusion.

The next propositions are essential for our analysis. In fact several results of this paper, including Theorems 3.6, 5.2 and 8.10, depend on these results.

Fact 2.15 ([Y4, Theorem 3.1]). Let $\Phi:=\Phi_{Y \rightarrow X}^{\mathbf{E}}: \mathbf{D}(Y) \rightarrow \mathbf{D}(X)$ be a FMT. We denote by $\Psi$ this functor $\Phi$ or the composition $\mathcal{D}_{X} \circ \Phi$. Let $w$ be a primitive Mukai vector on $Y$ with $\left\langle w^{2}\right\rangle>0$. Let $H^{\prime}$ be a general ample divisor on $Y$ with respect to $w$. Set $v:= \pm \Psi(w)$. Let $H$ be a general ample divisor on $X$ with respect to $v$. 
If $\Psi(E)$ is not a sheaf up to shift for all $E \in M_{Y}^{H^{\prime}}(w)$, then there is an integer $k$ such that for a general $E \in M_{Y}^{H^{\prime}}(w), \Psi(E)$ fits in an exact triangle

$$
A_{0} \rightarrow \Psi(E) \rightarrow A_{1}[-1] \rightarrow A_{0}[1]
$$

in $\mathbf{D}(X)$ or $\mathbf{D}(X)_{\mathrm{op}}$. Here $A_{0}$ and $A_{1}$ are semi-homogeneous sheaves with

$$
v\left(A_{i}\right)=\ell_{i} v_{i},\left\langle v_{0}^{2}\right\rangle=\left\langle v_{1}^{2}\right\rangle=0,\left(\ell_{0}-1\right)\left(\ell_{1}-1\right)=0 .
$$

Remark 2.16. In [Y4, Theorem 3.1] only the statement for $\Phi$ is proved, but the proof works in this general situation.

Fact 2.17 ([Y4, Lemma 3.2]). Keep notations in Fact 2.15.

(1) If $\Psi\left(E_{0}\right) \in \operatorname{Coh}(X)$ for an element $E_{0} \in M_{Y}^{H^{\prime}}(w)$, then $\Psi(E)$ is stable with respect to $H$ for a general $E \in M_{Y}^{H^{\prime}}(w)$.

(2) If $V^{\bullet}:=\Psi(E)$ is not a sheaf for all $E \in M_{Y}^{H^{\prime}}(w)$, then there is an equivalence $\mathcal{F}: \mathbf{D}(X) \rightarrow$ $\mathbf{D}(X)$ or $\mathbf{D}(X) \rightarrow \mathbf{D}(X)_{\text {op }}$ such that $\mathcal{F}(v)=v$ and for a general $E \in M_{Y}^{H^{\prime}}(w)$ one of the following three conditions holds.

(a) $\operatorname{rk}\left(H^{0}\left(\mathcal{F}\left(V^{\bullet}\right)\right)\right)+\operatorname{rk}\left(H^{1}\left(\mathcal{F}\left(V^{\bullet}\right)\right)\right)<\operatorname{rk}\left(H^{0}\left(V^{\bullet}\right)\right)+\operatorname{rk}\left(H^{1}\left(V^{\bullet}\right)\right)$, or

(b) $\operatorname{deg} H^{1}\left(\mathcal{F}\left(V^{\bullet}\right)\right)<\operatorname{deg} H^{1}\left(V^{\bullet}\right)$ if $\operatorname{rk}\left(H^{1}\left(V^{\bullet}\right)\right)=0$, or

(c) $H^{1}\left(\mathcal{F}\left(V^{\bullet}\right)\right)=0$ is of 0 -dimensional.

\section{Semi-homogeneous Presentation of Sheaves}

We introduce the notion of semi-homogeneous presentations and discuss their properties. We show the uniqueness of this presentation in Proposition 3.3 and state the numerical criteria for the existence in Theorem 3.6. Its proof requires certain moduli of simple complexes, so we defer it to the next $\S$.

In this section, $X$ always means an abelian surface.

\subsection{Definitions and basic properties.}

Definition 3.1. For $E \in \operatorname{Coh}(X)$, a semi-homogeneous presentation is an exact sequence

$$
0 \rightarrow E \rightarrow E_{1} \rightarrow E_{2} \rightarrow 0 \quad \text { or } \quad 0 \rightarrow E_{1} \rightarrow E_{2} \rightarrow E \rightarrow 0
$$

with the condition $v\left(E_{1}\right)=\ell_{1} v_{1}, v\left(E_{2}\right)=\ell_{2} v_{2}$ and

$$
\begin{aligned}
& \ell_{1}, \ell_{2} \in \mathbb{Z}_{>0}, \quad\left(\ell_{1}-1\right)\left(\ell_{2}-1\right)=0, \\
& \left\langle v_{1}, v_{2}\right\rangle=-1, \quad v_{1}, v_{2}: \text { positive, primitive and isotropic Mukai vectors. }
\end{aligned}
$$

The former sequence is called a kernel presentation, and the latter is called a cokernel presentation.

For a Mukai vector $v$, the equation

$$
\begin{aligned}
& v= \pm\left(\ell_{2} v_{2}-\ell_{1} v_{1}\right), \\
& \ell_{1}, \ell_{2} \in \mathbb{Z}_{>0}, \quad\left(\ell_{1}-1\right)\left(\ell_{2}-1\right)=0, \\
& v_{1}, v_{2}: \text { positive, primitive and isotropic Mukai vectors with } \\
& \left\langle v_{1}, v_{2}\right\rangle=-1, \quad \mu\left(v_{1}\right)<\mu\left(v_{2}\right)
\end{aligned}
$$

is called the numerical equation. A solution $\left(v_{1}, v_{2}, \ell_{1}, \ell_{2}\right)$ of this equation (3.2) is called numerical solution of $v$.

Existence of semi-homogeneous presentation is not obvious a priori. We will show in Theorem 3.6 that numerical conditions on sheaves control the existence. The next proposition shows that a semi-homogeneous presentation for a stable sheaf $E$ guarantees the existence of a good FMT under which $E$ is transformed into the ideal sheaf of 0-dimensional subscheme. 
Proposition 3.2. Assume $\mathrm{NS}(X)=\mathbb{Z} H$. Let $\ell \in \mathbb{Z}_{>0}$ and $E$ be a stable sheaf on $X$ with $\left\langle v(E)^{2}\right\rangle=2 \ell$ and write the four possible semi-homogeneous presentations for $E$ as

\begin{tabular}{|c|c|c|c|c|c|c|c|c|}
\hline (1) & 0 & $\rightarrow$ & $E_{1}$ & $\rightarrow$ & $E_{2}$ & $\rightarrow$ & $E$ & $\rightarrow$ \\
\hline (2) & 0 & $\rightarrow$ & $E_{2}$ & $\rightarrow$ & $E_{1}$ & $\rightarrow$ & $E$ & $\rightarrow$ \\
\hline (3) & 0 & $\rightarrow$ & $E$ & $\rightarrow$ & $E_{2}$ & $\rightarrow$ & $E_{1}$ & $\rightarrow$ \\
\hline 4) & 0 & $\rightarrow$ & $E$ & $\rightarrow$ & $E_{1}$ & $\rightarrow$ & $E_{2}$ & $\rightarrow$ \\
\hline
\end{tabular}

Here $E_{1}$ is a simple semi-homogeneous sheaf with $v\left(E_{1}\right)=v_{1}$ and $E_{2}$ is a semi-homogeneous sheaf with $v\left(E_{2}\right)=\ell v_{2}$, where $v_{1}$ and $v_{2}$ are primitive Mukai vectors.

Let $\mathbf{E}$ be a universal family of semi-homogeneous sheaves with Mukai vector $v_{2}$ and set $Y:=M_{X}^{H}\left(v_{2}\right), \Phi_{1}:=\Phi_{X \rightarrow Y}^{\mathbf{E}}, \Phi_{2}:=\Phi_{X \rightarrow Y}^{\mathbf{E}^{\vee}}$. Finally let $\mathcal{D}_{X}: \mathbf{D}(X) \rightarrow \mathbf{D}(X)_{\text {op }}$ be the dualizing functor and $\widehat{\Phi}_{1}:=\Phi_{1} \circ \mathcal{D}_{X}$ be the composition.

Consider the conditions

$$
\begin{aligned}
& \mathrm{WIT}_{i} \text { holds for } E \text { w.r.t. } \widehat{\Phi}_{1} \text { and } \widehat{\Phi}_{1}^{i}(E) \cong L \otimes I_{Z}, \\
& \mathrm{WIT}_{i} \text { holds for } E \text { w.r.t. } \Phi_{2} \text { and } \Phi_{2}^{i}(E) \cong L \otimes I_{Z},
\end{aligned}
$$

where $Z$ is a 0 -dimensional subscheme of length $\ell, I_{Z}$ is its ideal sheaf and $L$ is a line bundle on $Y$. Then the existence of each presentation is equivalent to the following condition.

$$
(1) \Longleftrightarrow(\# 21), \quad(2) \Longleftrightarrow(\# 12), \quad(3) \Longleftrightarrow(\# 11), \quad(4) \Longleftrightarrow(\# 22)
$$

Moreover, the condition that a stable sheaf on $X$ has a semi-homogeneous presentation is an open condition.

Proof. This is the consequence of Lemma 4.5, which will be given in $\S 4$.

Proposition 3.3. Suppose $\operatorname{NS}(X)=\mathbb{Z} H$. Let $E$ be a coherent sheaf on $X$ with $\left\langle v(E)^{2}\right\rangle>0$. Then a kernel (or cokernel) presentation of $E$ is unique, if it exists.

More generally, we have the next statements.

Lemma 3.4. Suppose $\operatorname{NS}(X)=\mathbb{Z} H$. Let $E$ be a coherent sheaf on $X$ with $\left\langle v(E)^{2}\right\rangle>0$.

(1) Suppose that we are given two exact sequences containing $E$.

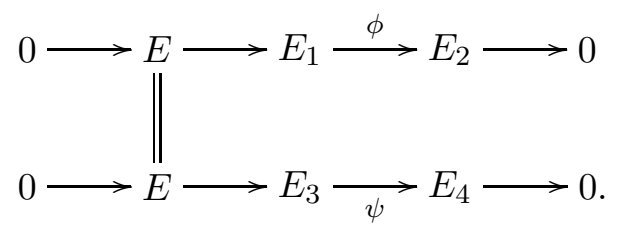

Here $E_{i}$ 's are semi-homogeneous sheaves and neither $\phi$ nor $\psi$ is trivial.

Then $E_{1} \cong E_{3}, E_{2} \cong E_{4}$ and the diagram extends to the next commuting diagram.

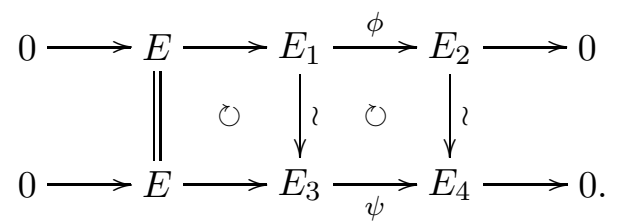

(2) Suppose that we are given two exact sequences containing $E$.

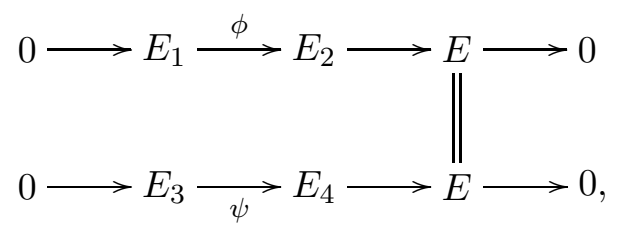


where $E_{i}$ are semi-homogeneous sheaves and neither $\phi$ nor $\psi$ is trivial. Then we have $E_{1} \cong E_{3}$ and $E_{2} \cong E_{4}$. We also have the next commuting diagram.

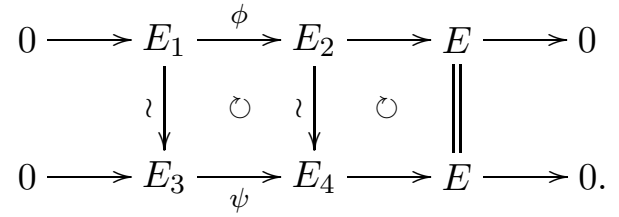

Proof. This is the corollary of the next Lemma 3.5.

Lemma 3.5. Assume $\mathrm{NS}(X)=\mathbb{Z} H$. Let $E^{\bullet}$ be a bounded complex on $X$ such that $\left\langle v\left(E^{\bullet}\right)^{2}\right\rangle>$ 0 . Assume that $E^{\bullet}$ has two expressions

$$
E^{\bullet} \cong\left[E_{1} \stackrel{\phi}{\rightarrow} E_{2}\right] \cong\left[F_{1} \stackrel{\psi}{\rightarrow} F_{2}\right],
$$

where $E_{1}, E_{2}, F_{1}, F_{2}$ are semi-homogeneous sheaves and $\phi, \psi$ are non-trivial. Then there are isomorphisms $f_{i}: E_{i} \cong F_{i}$ which induce the following commutative diagram in $\mathbf{D}(X)$.

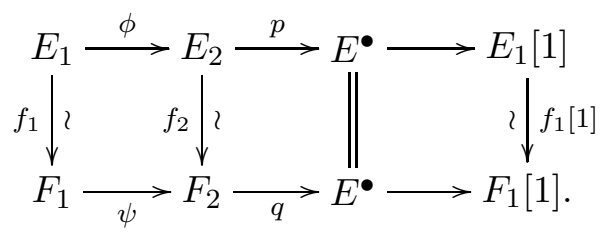

Proof. The proof is divided into several steps.

Step 1. $\mu\left(E_{1}\right)<\mu\left(E_{2}\right), \mu\left(F_{1}\right)<\mu\left(F_{2}\right)$ and $\operatorname{Ext}^{1}\left(F_{2}, E_{1}\right)=\operatorname{Ext}^{1}\left(E_{2}, F_{1}\right)=0$.

Note that

$$
v\left(E^{\bullet}\right)=v\left(E_{2}\right)-v\left(E_{1}\right)=v\left(F_{2}\right)-v\left(F_{1}\right) .
$$

Then we see that $0<\left\langle v\left(E^{\bullet}\right)^{2}\right\rangle=-2\left\langle v\left(E_{1}\right), v\left(E_{2}\right)\right\rangle=-2\left\langle v\left(F_{1}\right), v\left(F_{2}\right)\right\rangle$. Thus $\left\langle v\left(E_{1}\right), v\left(E_{2}\right)\right\rangle=$ $\left\langle v\left(F_{1}\right), v\left(F_{2}\right)\right\rangle<0$. We also find from (3.6) that

$$
\left\langle v\left(E_{1}\right), v\left(F_{2}\right)\right\rangle=\left\langle v\left(E_{1}\right), v\left(E_{2}\right)+v\left(F_{1}\right)-v\left(E_{1}\right)\right\rangle=\left\langle v\left(E_{1}\right), v\left(E_{2}\right)\right\rangle+\left\langle v\left(E_{1}\right), v\left(F_{1}\right)\right\rangle .
$$

From Lemma 2.13 and the inequality $\left\langle v\left(E_{1}\right), v\left(E_{2}\right)\right\rangle<0$, we find that $\left\langle v\left(E_{1}\right), v\left(F_{2}\right)\right\rangle<0$. In the same way, we have $\left\langle v\left(F_{1}\right), v\left(E_{2}\right)\right\rangle<0$. Then Fact 2.12 shows that $\operatorname{Ext}^{1}\left(F_{2}, E_{1}\right)=\operatorname{Ext}^{1}\left(E_{2}, F_{1}\right)=$ 0 .

Lemma 2.13 and $\left\langle v\left(E_{1}\right), v\left(E_{2}\right)\right\rangle<0$ also implies $\mathbb{Q} v\left(E_{1}\right) \neq \mathbb{Q} v\left(E_{2}\right)$. Then since $\phi \neq 0$, we have $\mu\left(E_{1}\right)<\mu\left(E_{2}\right)$. Similarly we have $\mu\left(F_{1}\right)<\mu\left(F_{2}\right)$.

Step 2. There exist morphisms $f_{2}: E_{2} \rightarrow F_{2}$ and $g_{2}: F_{2} \rightarrow E_{2}$ which make the next diagram commutative.

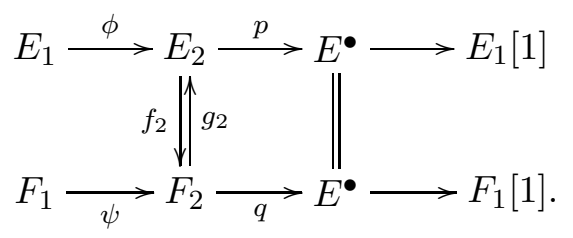

By assumption we have the next exact sequence.

$$
\operatorname{Hom}\left(E_{2}, F_{2}\right) \rightarrow \operatorname{Hom}\left(E_{2}, E^{\bullet}\right) \rightarrow \operatorname{Ext}^{1}\left(E_{2}, F_{1}\right) .
$$

By Step 1 the last term vanishes. Therefore any morphism $F_{2} \rightarrow E^{\bullet}$ lifts to $E_{2} \rightarrow F_{2}$. Since we are given a non-zero morphism $q: F_{2} \rightarrow E^{\bullet}$, there exists a non-zero morphism $f_{2}: E_{2} \rightarrow F_{2}$. It is easy to verify that the resulting diagram is commutative. By the symmetry of the conditions, there exists a non-zero morphism $g_{2}: F_{2} \rightarrow E_{2}$ which makes the resulting diagram commutative. Thus we have the diagram (3.7). 
Step 3. $f_{2}$ is an isomorphism. We also have an isomorphism $E_{1} \stackrel{\sim}{\rightarrow} F_{1}$ and the resulting diagram 3.5 is commutative.

Let $h:=g_{2} \circ f_{2}-\operatorname{id}_{E_{2}} \in \operatorname{End}\left(E_{2}\right)$. Then the commutativity of the diagram (3.7) yields $p \circ h=0$, so that $h$ induces $\widetilde{h}: E_{2} \rightarrow E_{1}$. Hence from $\mu\left(E_{1}\right)<\mu\left(E_{2}\right)$ (Step 1) and the semi-stability of $E_{i}$, we have $\operatorname{Hom}\left(E_{2}, E_{1}\right)=0$. Therefore $\widetilde{h}=0$, so that $h=0$. Then $g_{2} \circ f_{2}=\mathrm{id}_{E_{2}}$. By the symmetry of the condition, we also have $f_{2} \circ g_{2}=\mathrm{id}_{F_{2}}$. Thus $f_{2}$ is an isomorphism.

The second statement is shown by routine procedures.

3.2. Numerical criterion of semi-homogeneous presentation. Now we state the numerical criterion for the existence of semi-homogeneous presentation. Fix an abelian surface $X$.

Theorem 3.6. Let $\operatorname{NS}(X)=\mathbb{Z} H$ and $v$ be a Mukai vector with $\left\langle v^{2}\right\rangle>0$.

(1) If there is at least two numerical solutions for $v$, then a general member of $M_{X}^{H}(v)$ has both kernel presentation and cokernel presentation. Each presentation is unique.

(2) If there is only one numerical solution for $v$, then a general member of $M_{X}^{H}(v)$ has either kernel presentation or cokernel presentation. Such a presentation is unique.

The proof will be presented in $\S \S 4.3$.

\section{Moduli of Simple COMPLEXes OF NUmerical inVARIANT $\left(v_{1}, v_{2}, \ell_{1}, \ell_{2}\right)$}

In this section we construct fine moduli spaces of simple complexes, which will be used in the proof of Theorem 3.6. We fix an abelian surface $X$ with $\operatorname{NS}(X)=\mathbb{Z} H$.

4.1. Complexes associated to numerical solutions. In this subsection we fix a positive Mukai vector $v \in H^{\mathrm{ev}}(X, \mathbb{Z})$ alg with $\ell:=\left\langle v^{2}\right\rangle / 2 \in \mathbb{Z}_{>0}$.

Let $\left(v_{1}, v_{2}, \ell_{1}, \ell_{2}\right)$ be a numerical solution of $v$. For $i=1,2$, let $Y_{i}:=M_{X}^{H}\left(v_{i}\right)$ and $\mathbf{E}_{i}$ be a universal family such that $v\left(\Phi_{X \rightarrow Y_{i}}^{\mathbf{E}_{i}^{\vee}}\left(v_{j}\right)\right)=(1,0,0), j \neq i$. By Lemma 2.7. $\mathrm{NS}\left(Y_{i}\right)$ is of rank one. We define $H_{i}$ to be the ample generator of $\operatorname{NS}\left(Y_{i}\right)$. We also set $\mathbf{P}:=\mathbf{R} p_{Y_{1} \times Y_{2} *}\left(\mathbf{E}_{1}^{\vee} \otimes_{\mathcal{O}_{X}} \mathbf{E}_{2}\right)$, where $p_{Y_{1} \times Y_{2}}: Y_{1} \times X \times Y_{2} \rightarrow Y_{1} \times Y_{2}$ is the projection. Then $\mathbf{P}$ is a line bundle on $Y_{1} \times Y_{2}$ with $\left.\mathbf{P}\right|_{\left\{y_{1}\right\} \times Y_{2}} \in \operatorname{Pic}^{0}\left(Y_{2}\right)$ and $\left.\mathbf{P}\right|_{Y_{1} \times\left\{y_{2}\right\}} \in \operatorname{Pic}^{0}\left(Y_{1}\right)$. It is easy to see that $Y_{2} \cong \widehat{Y}_{1}$ and $\mathbf{P}$ is the Poincaré line bundle on $Y_{1} \times Y_{2}$.

For each numerical solution, we will construct families of complexes with Mukai vector $\ell_{2} v_{2}-$ $\ell_{1} v_{1}$ parametrized by the moduli spaces of rank 1 sheaves $Y_{j} \times \operatorname{Hilb}^{\ell}\left(Y_{i}\right)$. Here the indices $\{i, j\}=\{1,2\}$ are defined to be $\left(\ell_{i}, \ell_{j}\right)=(\ell, 1)$. We start with characterizations of our complexes.

Lemma 4.1. Let $V^{\bullet}$ be a bounded complex on $X$. The following three conditions are equivalent.

(1) $V^{\bullet}$ is simple, that is, $\operatorname{Hom}\left(V^{\bullet}, V^{\bullet}\right) \cong \mathbb{C}$, and $H^{i}\left(V^{\bullet}\right)=0$ for $i \neq-1,0, H^{-1}\left(V^{\bullet}\right) \in$ $M_{X}^{H}\left(\ell_{1} v_{1}\right)^{s s}$ and $H^{0}\left(V^{\bullet}\right) \in M_{X}^{H}\left(\ell_{2} v_{2}\right)^{s s}$.

(2) $\Phi_{X \rightarrow Y_{1}}^{\mathbf{E}_{1}^{\vee}}\left(V^{\bullet}\right) \in M_{Y_{1}}^{H_{1}}\left(\ell_{2}, 0,-\ell_{1}\right)$.

(3) $\Phi_{X \rightarrow Y_{2}}^{\mathbf{E}_{2}}\left(\left(V^{\bullet}\right)^{\vee}[1]\right) \in M_{Y_{2}}^{H_{2}}\left(\ell_{1}, 0,-\ell_{2}\right)$.

Proof. (a) We first prove the equivalence of (2) and (3). From $\Phi_{Y_{2} \rightarrow Y_{1}}^{\mathbf{P}}=\Phi_{X \rightarrow Y_{1}}^{\mathbf{E}_{1}^{\vee}} \Phi_{Y_{2} \rightarrow X}^{\mathbf{E}_{2}}$, we find that $\Phi_{X \rightarrow Y_{2}}^{\mathbf{E}_{2}^{\vee[2]}}=\Phi_{Y_{1} \rightarrow Y_{2}}^{\mathbf{P}^{\vee}[2]} \Phi_{X \rightarrow Y_{1}}^{\mathbf{E}_{1}^{\vee}}$. Then Lemma 2.8 yields that

$$
\Phi_{X \rightarrow Y_{2}}^{\mathbf{E}_{2}} \mathcal{D}_{X}=\mathcal{D}_{Y_{2}} \Phi_{X \rightarrow Y_{2}}^{\mathbf{E}_{2}^{\vee}[2]}=\mathcal{D}_{Y_{2}} \Phi_{Y_{1} \rightarrow Y_{2}}^{\mathbf{P}^{\vee}[2]} \Phi_{X \rightarrow Y_{1}}^{\mathbf{E}_{1}^{\vee}}=\Phi_{Y_{1} \rightarrow Y_{2}}^{\mathbf{P}} \mathcal{D}_{Y_{1}} \Phi_{X \rightarrow Y_{1}}^{\mathbf{E}_{1}^{\vee}}
$$

Hence

$$
\Phi_{Y_{1} \rightarrow Y_{2}}^{\mathbf{P}[1]} \mathcal{D}_{Y_{1}}\left(\Phi_{X \rightarrow Y_{1}}^{\mathbf{E}_{1}^{\vee}}\left(V^{\bullet}\right)\right)=\Phi_{X \rightarrow Y_{2}}^{\mathbf{E}_{2}}\left(\left(V^{\bullet}\right)^{\vee}[1]\right) .
$$

Then the equivalence follows from Lemma 4.3 below. 
(b) Next we prove the statement $(1) \Rightarrow(2)$ (or (3)). Assume that $V^{\bullet}$ satisfies (1). Then we have an exact triangle

$$
H^{-1}\left(V^{\bullet}\right)[1] \rightarrow V^{\bullet} \rightarrow H^{0}\left(V^{\bullet}\right) \rightarrow H^{-1}\left(V^{\bullet}\right)[2]
$$

Since $V^{\bullet}$ is simple, we have $\operatorname{Hom}\left(H^{0}\left(V^{\bullet}\right), H^{-1}\left(V^{\bullet}\right)[2]\right) \neq 0$. Since $\Phi_{X \rightarrow Y_{1}}^{\mathbf{E}_{1}^{\vee}}\left(H^{-1}\left(V^{\bullet}\right)[2]\right) \in$ $M_{Y_{1}}^{H_{1}}\left(0,0, \ell_{1}\right)^{s s}$ and $\Phi_{X \rightarrow Y_{1}}^{\mathbf{E}_{1}^{\vee}}\left(H^{0}\left(V^{\bullet}\right)\right) \in M_{Y_{1}}^{H_{1}}\left(\ell_{2}, 0,0\right)^{s s}, H^{i}\left(\Phi_{X \rightarrow Y_{1}}^{\mathbf{E}_{1}^{\vee}}\left(V^{\bullet}\right)\right)=0$ for $i \neq 0,1$ and we have an exact sequence

$$
0 \rightarrow H^{0}\left(\Phi_{X \rightarrow Y_{1}}^{\mathbf{E}_{1}^{\vee}}\left(V^{\bullet}\right)\right) \rightarrow \Phi_{X \rightarrow Y_{1}}^{\mathbf{E}_{1}^{\vee}}\left(H^{0}\left(V^{\bullet}\right)\right) \rightarrow \Phi_{X \rightarrow Y_{1}}^{\mathbf{E}_{1}^{\vee}}\left(H^{-1}\left(V^{\bullet}\right)[2]\right) \rightarrow H^{1}\left(\Phi_{X \rightarrow Y_{1}}^{\mathbf{E}_{1}^{\vee}}\left(V^{\bullet}\right)\right) \rightarrow 0 .
$$

We will prove that $H^{1}\left(\Phi_{X \rightarrow Y_{1}}^{\mathbf{E}_{1}^{\vee}}\left(V^{\bullet}\right)\right)=0$ by showing $\operatorname{Hom}\left(\left.\mathbf{E}_{1}\right|_{\{y\} \times X}, V^{\bullet}[1]\right)=0$ for all $y \in Y_{1}$. Assume that $\operatorname{Hom}\left(\left.\mathbf{E}_{1}\right|_{\{y\} \times X}, V^{\bullet}[1]\right) \neq 0$ for a point $y \in Y_{1}$. Then the Serre duality implies that $\operatorname{Hom}\left(V^{\bullet},\left.\mathbf{E}_{1}\right|_{\{y\} \times X}[1]\right) \neq 0$. Since $\left\langle v\left(H^{0}\left(V^{\bullet}\right)\right), v_{1}\right\rangle=-1$, Fact 2.12 implies that $\operatorname{Hom}\left(H^{0}\left(V^{\bullet}\right),\left.\mathbf{E}_{1}\right|_{\{y\} \times X}[1]\right)=0$. Hence we have a morphism $\varphi:\left.V^{\bullet} \rightarrow \mathbf{E}_{1}\right|_{\{y\} \times X}[1]$ such that $H^{-1}\left(V^{\bullet}\right) \rightarrow H^{-1}\left(\left.\mathbf{E}_{1}\right|_{\{y\} \times X}[1]\right)$ is surjective in $\operatorname{Coh}(X)$. By Lemma 4.2 below and the semi-stability, there is an injective homomorphism $\left.\mathbf{E}_{1}\right|_{\{y\} \times X} \rightarrow H^{-1}\left(V^{\bullet}\right)$. Hence we have a morphism $\left.V^{\bullet} \rightarrow \mathbf{E}_{1}\right|_{\{y\} \times X}[1] \rightarrow H^{-1}\left(V^{\bullet}\right)[1] \rightarrow V^{\bullet}$ which induces a non-zero homomorphism $H^{-1}\left(V^{\bullet}\right) \rightarrow H^{-1}\left(\left.\mathbf{E}_{1}\right|_{\{y\} \times X}[1]\right) \rightarrow H^{-1}\left(V^{\bullet}\right)$ and the zero map $H^{0}\left(V^{\bullet}\right) \rightarrow H^{0}\left(V^{\bullet}\right)$. Since $V^{\bullet}$ is simple, this is impossible. Therefore $\operatorname{Hom}\left(\left.\mathbf{E}_{1}\right|_{\{y\} \times X}, V^{\bullet}[1]\right)=0$ for all $y \in Y_{1}$.

By (4.1), $\Phi_{X \rightarrow Y_{1}}^{\mathbf{E}_{1}^{\vee}}\left(V^{\bullet}\right)$ is a $\mu$-semi-stable sheaf with Mukai vector $\left(\ell_{2}, 0,-\ell_{1}\right)$. We will prove that $\Phi_{X \rightarrow Y_{1}}^{\mathbf{E}_{1}^{\vee}}\left(V^{\bullet}\right)$ is stable. The claim for $\ell_{2}=1$ is trivial. Hence we assume that $\left(\ell_{1}, \ell_{2}\right)=(1, \ell)$. Assume that there is a stable subsheaf $F$ of $\Phi_{X \rightarrow Y_{1}}^{\mathbf{E}_{1}^{\vee}}\left(V^{\bullet}\right)$ such that

$$
\operatorname{rk}(F)<\ell, \quad c_{1}(F)=0, \quad \chi(F) / \operatorname{rk} F \geq-1 / \ell .
$$

Set $v(F)=(r, 0, a)$. Then since $\left\langle v(F)^{2}\right\rangle / 2=-r a \geq 0$, we have $a=0$, which yields $F \in$ $M_{Y_{1}}^{H_{1}}(1,0,0)$.

Since $F$ is a subsheaf of a semi-homogeneous sheaf $\Phi_{X \rightarrow Y_{1}}^{\mathbf{E}_{1}^{\vee}}\left(H^{0}\left(V^{\bullet}\right)\right)$ with $v(F)=(\ell, 0,0)$, we find from Lemma 4.2 below that $\operatorname{Hom}\left(\Phi_{X \rightarrow Y_{1}}^{\mathbf{E}_{1}^{\vee}}\left(H^{0}\left(V^{\bullet}\right)\right), F\right) \neq 0$. Then we see that $\Phi_{X \rightarrow Y_{1}}^{\mathbf{E}_{1}^{\vee}}\left(V^{\bullet}\right)$ is not simple. Therefore $\Phi_{X \rightarrow Y_{1}}^{\mathbf{E}_{1}^{\vee}}\left(V^{\bullet}\right)$ is stable. Thus (2) holds.

(c) Finally we prove the statement $(2) \Rightarrow(1)$. Assume that (2) (and hence (3)) holds.

We first assume that $\ell_{2}=1$. Then $\Phi_{X \rightarrow Y_{1}}^{\mathbf{E}_{1}^{\vee}}\left(V^{\bullet}\right) \cong L \otimes I_{Z}$ has the kernel presentation

$$
0 \rightarrow L \otimes I_{Z} \rightarrow L \rightarrow L \otimes \mathcal{O}_{Z} \rightarrow 0
$$

where $L \in \operatorname{Pic}^{0}\left(Y_{1}\right)$ and $I_{Z} \in \operatorname{Hilb}^{\ell}\left(Y_{1}\right)$. Applying $\Phi_{Y_{1} \rightarrow X}^{\mathbf{E}_{1}}$ to this exact sequence, we get

$$
H^{i}\left(V^{\bullet}\right)=0(i \neq-1,0), \quad H^{-1}\left(V^{\bullet}\right)=\Phi_{Y_{1} \rightarrow X}^{\mathbf{E}_{1}[1]}\left(L \otimes \mathcal{O}_{Z}\right), \quad H^{0}\left(V^{\bullet}\right)=\Phi_{Y_{1} \rightarrow X}^{\mathbf{E}_{1}[2]}(L) .
$$

Thus (1) holds.

If $\ell_{1}=1$, then $\Phi_{X \rightarrow Y_{2}}^{\mathbf{E}_{2}}\left(\left(V^{\bullet}\right)^{\vee}[1]\right) \cong L \otimes I_{Z}$ has the kernel presentation

$$
0 \rightarrow L \otimes I_{Z} \rightarrow L \rightarrow L \otimes \mathcal{O}_{Z} \rightarrow 0
$$

where $L \in \operatorname{Pic}^{0}\left(Y_{2}\right)$ and $I_{Z} \in \operatorname{Hilb}^{\ell}\left(Y_{2}\right)$. Applying $\mathcal{D}_{X} \Phi_{Y_{2} \rightarrow X}^{\mathbf{E}_{2}^{\vee}}=\Phi_{Y_{2} \rightarrow X}^{\mathbf{E}_{2}[2]} \mathcal{D}_{Y_{2}}$ (see Lemma 2.8) to this exact sequence, we get

$$
H^{i}\left(V^{\bullet}\right)=0(i \neq-1,0), \quad H^{-1}\left(V^{\bullet}\right)=\Phi_{Y_{2} \rightarrow X}^{\mathbf{E}_{2}[2]}\left(\left(L \otimes \mathcal{O}_{Z}\right)^{\vee}\right), \quad H^{0}\left(V^{\bullet}\right)=\Phi_{Y_{2} \rightarrow X}^{\mathbf{E}_{2}[2]}\left(L^{\vee}\right) .
$$

Thus (1) holds again in this case. 
Lemma 4.2. Let $E$ be a simple semi-homogeneous sheaf and $F$ a semi-homogeneous sheaf with $v(F)=\ell v(E), \ell \in \mathbb{Z}_{>0}$. Then $\operatorname{Hom}(E, F) \neq 0$ if and only if $\operatorname{Hom}(F, E) \neq 0$.

Proof. See [M1, Proposition 6.10].

Lemma 4.3. The functors $\Phi_{Y_{1} \rightarrow Y_{2}}^{\mathbf{P}[1]} \mathcal{D}_{Y_{1}}$ and $\Phi_{Y_{1} \rightarrow Y_{2}}^{\mathbf{P}^{\vee}[1]} \mathcal{D}_{Y_{1}}$ induce an isomorphism $M_{Y_{1}}^{H_{1}}(r, 0,-a) \cong$ $M_{Y_{2}}^{H_{2}}(a, 0,-r)$.

Proof. See [Y1, Theorem 8.4] or [M7, Corollary 4.5] for $a=1$.

Remark 4.4. $\Phi_{Y_{2} \rightarrow Y_{1}}^{\mathbf{P}[1]} \mathcal{D}_{Y_{2}} \Phi_{Y_{1} \rightarrow Y_{2}}^{\mathbf{P}[1]} \mathcal{D}_{Y_{1}} \cong 1_{Y_{1}}$. Hence $\Phi_{Y_{2} \rightarrow Y_{1}}^{\mathbf{P}[1]} \mathcal{D}_{Y_{2}}$ gives the inverse of $\Phi_{Y_{1} \rightarrow Y_{2}}^{\mathbf{P}[1]} \mathcal{D}_{Y_{1}}$.

Lemma 4.5. Let $V^{\bullet}$ be a bounded complex on $X$. The following three conditions are equivalent.

(1) $V^{\bullet}$ is simple and quasi-isomorphic to a complex $\left[U^{-1} \rightarrow U^{0}\right]$ with $U^{-1} \in M_{X}^{H}\left(\ell_{1} v_{1}\right)^{s s}$ and $U^{0} \in M_{X}^{H}\left(\ell_{2} v_{2}\right)^{s s}$.

(2) $\Phi_{X \rightarrow Y_{2}}^{\mathbf{E}_{2}^{\vee}}\left(V^{\bullet}[1]\right) \in M_{X}^{H}\left(\ell_{1}, 0,-\ell_{2}\right)$.

(3) $\mathcal{D}_{Y_{1}} \Phi_{X \rightarrow Y_{1}}^{\mathbf{E}_{1}^{\vee}}\left(V^{\bullet}\right)=\Phi_{X \rightarrow Y_{1}}^{\mathbf{E}_{1}}\left(\left(V^{\bullet}\right)^{\vee}[2]\right) \in M_{X}^{H}\left(\ell_{1}, 0,-\ell_{2}\right)$.

Proof. $\Phi_{Y_{2} \rightarrow Y_{1}}^{\mathbf{P}}=\Phi_{X \rightarrow Y_{1}}^{\mathbf{E}_{1}^{\vee}} \Phi_{Y_{2} \rightarrow X}^{\mathbf{E}_{2}}$ implies that $\Phi_{Y_{1} \rightarrow Y_{2}}^{\mathbf{P}_{\vee}}=\Phi_{X \rightarrow Y_{2}}^{\mathbf{E}_{2}^{\vee}} \Phi_{Y_{1} \rightarrow X}^{\mathbf{E}_{1}[2]}$. Using Lemma 2.8 we have

$$
\left(\Phi_{Y_{1} \rightarrow Y_{2}}^{\mathbf{P}^{\vee}} \mathcal{D}_{Y_{1}}\right) \Phi_{X \rightarrow Y_{1}}^{\mathbf{E}_{1}[2]} \mathcal{D}_{X}=\Phi_{Y_{1} \rightarrow Y_{2}}^{\mathbf{P}^{\vee}} \Phi_{X \rightarrow Y_{1}}^{\mathbf{E}_{1}^{\vee}}=\Phi_{X \rightarrow Y_{2}}^{\mathbf{E}_{2}^{\vee}} .
$$

Therefore Lemma 4.3 implies that (2) and (3) are equivalent.

The proof of the equivalence between (1) and (2) (or (3)) is similar to that in Lemma 4.1. Hence we only prove the direction $(1) \Rightarrow(2)$. Assume that (1) holds.

We will show that $\operatorname{Hom}\left(\left.\mathbf{E}_{2}\right|_{\{y\} \times X}, V^{\bullet}[2]\right)=0$ for all $y \in Y_{2}$. If $\operatorname{Hom}\left(V^{\bullet},\left.\mathbf{E}_{2}\right|_{\{y\} \times X}\right)=$ $\operatorname{Hom}\left(\left.\mathbf{E}_{2}\right|_{\{y\} \times X}, V^{\bullet}[2]\right)^{\vee} \neq 0$, then we have a non-zero homomorphism $\left.V^{0} \rightarrow \mathbf{E}_{2}\right|_{\{y\} \times X}$. By our choice of $v_{2}$, it is surjective. Then by Lemma 4.2, we also have an injective homomorphism $\left.\mathbf{E}_{2}\right|_{\{y\} \times X} \rightarrow V^{0}$, which gives a morphism $\left.\mathbf{E}_{2}\right|_{\{y\} \times X} \rightarrow V^{\bullet}$. Hence we have a non-zero morphism $\left.V^{\bullet} \rightarrow \mathbf{E}_{2}\right|_{\{y\} \times X} \rightarrow V^{\bullet}$. Obviously it is not isomorphic. By the simpleness of $V^{\bullet}$, it is impossible. Therefore $\operatorname{Hom}\left(\left.\mathbf{E}_{2}\right|_{\{y\} \times X}, V^{\bullet}[2]\right)=0$ for all $y \in Y_{2}$.

Then $\Phi_{X \rightarrow Y_{2}}^{\mathbf{E}_{2}^{\vee}}\left(\left(V^{\bullet}\right)^{\vee}[1]\right)$ is a simple sheaf. Then the same arguments as in Lemma 4.1 show the stability.

4.2. Existence and some properties of the moduli spaces. As in the last subsection, we fix a positive Mukai vector $v \in H^{\mathrm{ev}}(X, \mathbb{Z})_{\text {alg }}$ with $\ell:=\left\langle v^{2}\right\rangle / 2 \in \mathbb{Z}_{>0}$. For a numerical solution $\left(v_{1}, v_{2}, \ell_{1}, \ell_{2}\right)$ of $v$, we continue to use the symbols $Y_{i}, \mathbf{E}_{i}, H_{i}, \mathbf{P}$.

Let $\mathcal{Z}_{1} \subset \operatorname{Hilb}^{\ell}\left(Y_{1}\right) \times Y_{1}$ and $\mathcal{Z}_{2} \subset \operatorname{Hilb}^{\ell}\left(Y_{2}\right) \times Y_{2}$ be the universal family of $\ell$-points on $Y_{1}$ and $Y_{2}$ respectively. We set $\mathfrak{H}_{1}:=Y_{2} \times \operatorname{Hilb}^{\ell}\left(Y_{1}\right)$ and $\mathfrak{H}_{2}:=Y_{1} \times \operatorname{Hilb}^{\ell}\left(Y_{2}\right)$. Then we have an exact sequence on $\mathfrak{H}_{i} \times Y_{i}$ :

$$
0 \rightarrow \mathbf{P} \otimes I_{\mathcal{Z}_{i}} \rightarrow \mathbf{P} \rightarrow \mathbf{P} \otimes \mathcal{O}_{\mathcal{Z}_{i}} \rightarrow 0
$$

where $i=1,2$ and we simply denote the pull-backs of $\mathbf{P}, I_{\mathcal{Z}_{i}}$ and $\mathcal{O}_{\mathcal{Z}_{i}}$ by the same symbol. For $i=1,2$, we set $c(1):=2$ and $c(2):=1$. Applying $\Phi_{Y_{i} \rightarrow Y_{j}}^{\mathbf{P}[1]}$ to the dual of (4.2), we have an exact sequence on $\mathfrak{H}_{i} \times Y_{c(i)}$ :

$$
0 \rightarrow \Phi_{Y_{i} \rightarrow Y_{c(i)}}^{\mathbf{P}[1]}\left(\left(\mathbf{P} \otimes I_{\mathcal{Z}_{i}}\right)^{\vee}\right) \rightarrow \Phi_{Y_{i} \rightarrow Y_{c(i)}}^{\mathbf{P}[2]}\left(\left(\mathbf{P} \otimes \mathcal{O}_{\mathcal{Z}_{i}}\right)^{\vee}\right) \rightarrow \Phi_{Y_{i} \rightarrow Y_{c(i)}}^{\mathbf{P}[2]}\left(\mathbf{P}^{\vee}\right) \rightarrow 0 .
$$

Then for $i=1,2, \mathbf{P} \otimes I_{\mathcal{Z}_{i}}$ and $\Phi_{Y_{c(i)} \rightarrow Y_{i}}^{\mathbf{P}[1]}\left(\left(\mathbf{P} \otimes I_{\mathcal{Z}_{c(i)}}\right)^{\vee}\right)$ are universal families of stable sheaves on $Y_{i}$ with Mukai vectors $(1,0,-\ell)$ and $(\ell, 0,-1)$ respectively. $\left.\Phi_{Y_{c(i)} \rightarrow Y_{i}}^{\mathbf{P}^{\vee}[1]}\left(\mathbf{P} \otimes I_{\mathcal{Z}_{c(i)}}\right)^{\vee}\right)$ is also a universal family of stable sheaves on $Y_{i}$ with Mukai vector $(\ell, 0,-1)$. 
Remark 4.6. $\Phi_{Y_{c(i)} \rightarrow Y_{i}}^{\mathbf{P}[2]}\left(\mathbf{P}^{\vee}\right) \cong \mathcal{O}_{\Delta}$, where $\Delta \subset Y_{i} \times Y_{i}$ is the diagonal.

Applying $\Phi_{Y_{i} \rightarrow X}^{\mathbf{E}_{i}}$ to the exact sequences (4.2) and (4.3), we have the following families of simple complexes.

Proposition 4.7. For a numerical solution $\left(v_{1}, v_{2}, \ell_{1}, \ell_{2}\right)$ of $v$, we set the indices $i, j \in\{1,2\}$ by $\left(\ell_{i}, \ell_{j}\right)=(\ell, 1)$ and set $\mathfrak{S}:=\mathfrak{H}_{i}$.

(1) We have families of simple complexes $\mathcal{U}^{\bullet}=\left[\mathcal{U}^{-1} \rightarrow \mathcal{U}^{0}\right]$ of $v\left(\mathcal{U}_{s}^{\bullet}\right)=\ell_{2} v_{2}-\ell_{1} v_{1}, s \in \mathfrak{S}$ with the following properties.

(a) If $\ell_{1}=\ell$, we set $\mathcal{U}^{\bullet}:=\Phi_{Y_{1} \rightarrow X}^{\mathbf{E}_{1}[2]}\left(\mathbf{P} \otimes I_{\mathcal{Z}_{1}}\right)$. Then $H^{-1}\left(\mathcal{U}^{\bullet}\right)=\Phi_{Y_{1} \rightarrow X}^{\mathbf{E}_{1}}\left(\mathbf{P} \otimes \mathcal{O}_{\mathcal{Z}_{1}}\right)$ and $H^{0}\left(\mathcal{U}^{\bullet}\right)=\Phi_{Y_{1} \rightarrow X}^{\mathbf{E}_{1}[2]}(\mathbf{P})$ are semi-homogeneous sheaves with Mukai vectors $\ell v_{1}$ and $v_{2}$ respectively.

(b) If $\ell_{2}=\ell$, we set $\mathcal{U}^{\bullet}:=\Phi_{Y_{1} \rightarrow X}^{\mathbf{E}_{1}[2]} \Phi_{Y_{2} \rightarrow Y_{1}}^{\mathbf{P}[1]}\left(\left(\mathbf{P} \otimes I_{\mathcal{Z}_{2}}\right)^{\vee}\right)=\Phi_{Y_{2} \rightarrow X}^{\mathbf{E}_{2}[1]}\left(\left(\mathbf{P} \otimes I_{\mathcal{Z}_{2}}\right)^{\vee}\right)$. Then $H^{-1}\left(\mathcal{U}^{\bullet}\right)=\Phi_{Y_{2} \rightarrow X}^{\mathbf{E}_{2}}\left(\mathbf{P}^{\vee}\right)$ and $H^{0}\left(\mathcal{U}^{\bullet}\right)=\Phi_{Y_{2} \rightarrow X}^{\mathbf{E}_{2}[2]}\left(\left(\mathbf{P} \otimes \mathcal{O}_{\mathcal{Z}_{2}}\right)^{\vee}\right)$ are semi-homogeneous sheaves with Mukai vectors $v_{1}$ and $\ell v_{2}$ respectively.

(2) We have families of simple complexes $\mathcal{V}^{\bullet}: \mathcal{V}^{-1} \rightarrow \mathcal{V}^{0}$ of $v\left(\mathcal{V}_{s}^{\bullet}\right)=\ell_{2} v_{2}-\ell_{1} v_{1}, s \in \mathfrak{S}$ with the following properties.

(a) If $\ell_{2}=\ell$, we set $\mathcal{V}^{\bullet}:=\Phi_{Y_{2} \rightarrow X}^{\mathbf{E}_{2}[1]}\left(\mathbf{P} \otimes I_{\mathcal{Z}_{2}}\right)$. Then $\mathcal{V}^{-1}=\Phi_{Y_{2} \rightarrow X}^{\mathbf{E}_{2}}(\mathbf{P})$ and $\mathcal{V}^{0}=$ $\Phi_{Y_{2} \rightarrow X}^{\mathbf{E}_{2}}\left(\mathbf{P} \otimes \mathcal{O}_{\mathcal{Z}_{2}}\right)$ are families of the semi-homogeneous sheaves with Mukai vectors $v_{1}$ and $\ell v_{2}$ respectively.

(b) If $\ell_{1}=\ell$, we set $\mathcal{V}^{\bullet}=\Phi_{Y_{2} \rightarrow X}^{\mathbf{E}_{2}[1]} \Phi_{Y_{1} \rightarrow Y_{2}}^{\mathbf{P}^{\vee}[1]}\left(\left(\mathbf{P} \otimes I_{\mathcal{Z}_{1}}\right)^{\vee}\right)=\Phi_{Y_{1} \rightarrow X}^{\mathbf{E}_{1}[2]}\left(\left(\mathbf{P} \otimes I_{\mathcal{Z}_{1}}\right)^{\vee}\right)$. Then $\mathcal{V}^{-1}=\Phi_{Y_{1} \rightarrow X}^{\mathbf{E}_{1}}\left(\left(\mathbf{P} \otimes \mathcal{O}_{\mathcal{Z}_{1}}[2]\right)^{\vee}\right)$ and $\mathcal{V}^{0}=\Phi_{Y_{1} \rightarrow X}^{\mathbf{E}_{1}}\left(\mathbf{P}^{\vee}\right)$ are families of the semihomogeneous sheaves with Mukai vectors $\ell v_{1}$ and $v_{2}$ respectively.

Proposition 4.8. For a scheme $S$, let $V^{\bullet}$ be a family of simple complexes on $X$ such that each $V^{i}$ is flat over $S$.

(1) We set

$$
S^{\prime}:=\left\{\begin{array}{l|l}
s \in S & \begin{array}{l}
H^{i}\left(V_{s}^{\bullet}\right)=0(i \neq-1,0), \\
H^{-1}\left(V_{s}^{\bullet}\right) \in M_{X}^{H}\left(\ell_{1} v_{1}\right)^{s s}, H^{0}\left(V_{s}^{\bullet}\right) \in M_{X}^{H}\left(\ell_{2} v_{2}\right)^{s s}
\end{array}
\end{array}\right\} .
$$

Then $S^{\prime}$ is an open subset of $S$ and there is a unique morphism $f: S^{\prime} \rightarrow \mathfrak{S}$ such that $\left.\left(f \times 1_{X}\right)^{*}(\mathcal{U}) \cong V^{\bullet}\right|_{S^{\prime} \times X} \otimes p_{S^{\prime}}^{*}(N)$, where $N \in \operatorname{Pic}\left(S^{\prime}\right)$ and $p_{S^{\prime}}: S^{\prime} \times X \rightarrow S^{\prime}$ is the projection.

(2) We set

$$
S^{\prime}:=\left\{s \in S \mid V_{s}^{\bullet} \cong\left[W_{s}^{-1} \rightarrow W_{s}^{0}\right], W_{s}^{-1} \in M_{X}^{H}\left(\ell_{1} v_{1}\right)^{s s}, W_{s}^{0} \in M_{X}^{H}\left(\ell_{2} v_{2}\right)^{s s}\right\} .
$$

Then $S^{\prime}$ is an open subset of $S$ and we have a unique morphism $f: S^{\prime} \rightarrow \mathfrak{S}$ such that $\left.\left(f \times 1_{X}\right)^{*}(\mathcal{V}) \cong V^{\bullet}\right|_{S^{\prime} \times X} \otimes p_{S^{\prime}}^{*}(N)$, where $N \in \operatorname{Pic}\left(S^{\prime}\right)$ and $p_{S^{\prime}}: S^{\prime} \times X \rightarrow S^{\prime}$ is the projection.

Proof. (1) By Lemma 4.1, $S^{\prime}$ is the set of $s \in S$ such that $\Phi_{X \rightarrow Y_{1}}^{\mathbf{E}_{1}^{\vee}}\left(V^{\bullet}\right)_{s}$ is a stable sheaf with $v\left(\Phi_{X \rightarrow Y_{1}}^{\mathbf{E}_{1}^{\vee}}\left(V^{\bullet}\right)_{s}\right)=\left(\ell_{2}, 0,-\ell_{1}\right)$. By the openness of stability [HL, Proposition 2.3.1], $S^{\prime}$ is an open subset of $S$. Then we have a unique morphism $f: S^{\prime} \rightarrow \mathfrak{S}$ and a line bundle $N \in \operatorname{Pic}\left(S^{\prime}\right)$ such that $\left.\Phi_{X \rightarrow Y_{1}}^{\mathbf{E}_{1}^{\vee}}\left(V^{\bullet}\right)\right|_{S^{\prime} \times Y_{1}} \otimes p_{S^{\prime}}^{*}(N)$ is the pull-back of the universal family on $\mathfrak{H} \times Y_{1}$. Hence the claim holds by Proposition 4.7. The proof of (2) is similar.

By Proposition 4.8, the universal families $\mathcal{U}$ and $\mathcal{V}$ in Proposition 4.7 give moduli spaces of simple complexes. 
Theorem 4.9. Let $v$ be a positive Mukai vector with $\left\langle v^{2}\right\rangle>0$ and $\left(v_{1}, v_{2}, \ell_{1}, \ell_{2}\right)$ be a numerical solution of $v$.

(1) We have the fine moduli space $\mathfrak{M}^{-}\left(v_{1}, v_{2}, \ell_{1}, \ell_{2}\right)$ of simple complexes $V^{\bullet}$ such that $H^{i}\left(V^{\bullet}\right)=0, i \neq-1,0, H^{-1}\left(V^{\bullet}\right) \in M_{X}^{H}\left(\ell_{1} v_{1}\right)^{s s}$ and $H^{0}\left(V_{s}^{\bullet}\right) \in M_{X}^{H}\left(\ell_{2} v_{2}\right)^{s s}$.

(2) We have the fine moduli space $\mathfrak{M}^{+}\left(v_{1}, v_{2}, \ell_{1}, \ell_{2}\right)$ of simple complexes $V^{\bullet}$ such that $V^{\bullet} \cong$ $\left[W^{-1} \rightarrow W^{0}\right], W^{-1} \in M_{X}^{H}\left(\ell_{1} v_{1}\right)^{s s}, W^{0} \in M_{X}^{H}\left(\ell_{2} v_{2}\right)^{s s}$.

The following assertions show that $\mathfrak{M}^{ \pm}\left(v_{1}, v_{2}, \ell_{1}, \ell_{2}\right)$ behaves very well under the FourierMukai transforms.

Proposition 4.10. For a FMT $\Phi_{X \rightarrow Y}^{\mathbf{E}^{\vee}}, \mathbf{E} \in \operatorname{Coh}(X \times Y)$, we set $v_{i}^{\prime}:=\Phi_{X \rightarrow Y}^{\mathbf{E}^{\vee}}\left(v_{i}\right)(i=1,2)$. Then the following assertions hold.

(1) If $\mu\left(\left.\mathbf{E}\right|_{\{x\} \times Y}\right)<\mu\left(v_{1}\right)$ or $\mu\left(v_{2}\right) \leq \mu\left(\left.\mathbf{E}\right|_{\{x\} \times Y}\right)$, then we have an isomorphism

$$
\mathfrak{M}^{ \pm}\left(v_{1}, v_{2}, \ell_{1}, \ell_{2}\right) \stackrel{\sim}{\rightarrow} \mathfrak{M}^{ \pm}\left(v_{1}^{\prime}, v_{2}^{\prime}, \ell_{1}, \ell_{2}\right) .
$$

(2) If $\mu\left(v_{1}\right) \leq \mu\left(\mathbf{E}_{\mid\{x\} \times Y}\right)<\mu\left(v_{2}\right)$, then we have an isomorphism

$$
\mathfrak{M}^{ \pm}\left(v_{1}, v_{2}, \ell_{1}, \ell_{2}\right) \stackrel{\sim}{\rightarrow} \mathfrak{M}^{\mp}\left(v_{2}^{\prime}, v_{1}^{\prime}, \ell_{2}, \ell_{1}\right) .
$$

Proof. Let $E_{i}(i=1,2)$ be semi-homogeneous sheaves on $X$ with Mukai vectors $\ell_{i} v_{i}$. Then we have

$$
\begin{cases}\Phi_{X \rightarrow Y}^{\mathbf{E}^{\vee}}\left(E_{1}\right), \Phi_{X \rightarrow Y}^{\mathbf{E}^{\vee}}\left(E_{2}\right) \in \operatorname{Coh}(Y) & \mu\left(\left.\mathbf{E}\right|_{\{x\} \times Y}\right)<\mu\left(v_{1}\right), \\ \Phi_{X[2]}^{\mathbf{E}^{\vee}[2]}\left(E_{1}\right), \Phi_{X}^{\mathbf{E}^{\vee}}\left(E_{2}\right) \in \operatorname{Coh}(Y) & \mu\left(v_{1}\right) \leq \mu\left(\left.\mathbf{E}\right|_{\{x\} \times Y}\right)<\mu\left(v_{2}\right), \\ \Phi_{X \rightarrow Y}^{\mathbf{E}^{\vee}[2]}\left(E_{1}\right), \Phi_{X \rightarrow Y}^{\mathbf{E}^{\vee}[2]}\left(E_{2}\right) \in \operatorname{Coh}(Y) & \mu\left(v_{2}\right) \leq \mu\left(\mathbf{E}_{\mid\{x\} \times Y}\right) .\end{cases}
$$

From these results, we can easily prove the claim.

For $V^{\bullet} \in \mathfrak{M}^{+}\left(v_{1}, v_{2}, \ell_{1}, \ell_{2}\right)$, we set

$$
\Psi\left(V^{\bullet}\right):= \begin{cases}\Phi_{Y_{1} \rightarrow X}^{\mathbf{E}_{1}[2]} \mathcal{D}_{Y_{1}} \Phi_{X \rightarrow Y_{1}}^{\mathbf{E}_{1}^{\vee}}\left(V^{\bullet}\right) & \ell_{1}=\ell \\ \Phi_{Y_{2} \rightarrow X}^{\mathbf{E}_{2}[1]} \mathcal{D}_{Y_{2}} \Phi_{X \rightarrow Y_{2}}^{\mathbf{E}_{2}^{\vee}[1]}\left(V^{\bullet}\right) & \ell_{2}=\ell .\end{cases}
$$

Then Proposition 4.7 immediately implies the following.

Proposition 4.11. $\Psi$ induces an isomorphism

$$
\mathfrak{M}^{+}\left(v_{1}, v_{2}, \ell_{1}, \ell_{2}\right) \stackrel{\sim}{\rightarrow} \mathfrak{M}^{-}\left(v_{1}, v_{2}, \ell_{1}, \ell_{2}\right) .
$$

Proposition 4.12. For a numerical solution $\left(v_{1}, v_{2}, \ell_{1}, \ell_{2}\right)$ of $v$, we set

$$
k:= \begin{cases}0 & \ell_{2} v_{2}-\ell_{1} v_{1}>0, \\ 1 & \ell_{1} v_{1}-\ell_{2} v_{2}>0,\end{cases}
$$

and set

$$
\begin{aligned}
\mathfrak{M}^{+}\left(v_{1}, v_{2}, \ell_{1}, \ell_{2}\right)_{0} & :=\left\{V^{\bullet} \in \mathfrak{M}^{+}\left(v_{1}, v_{2}, \ell_{1}, \ell_{2}\right) \mid V^{\bullet}[k] \in M_{X}^{H}(v)\right\}, \\
M_{X}^{H}(v)_{0} & :=\left\{E \in M_{X}^{H}(v) \mid E[-k] \in \mathfrak{M}^{+}\left(v_{1}, v_{2}, \ell_{1}, \ell_{2}\right)\right\} .
\end{aligned}
$$

Then we have the following assertions.

(1) $\mathfrak{M}^{+}\left(v_{1}, v_{2}, \ell_{1}, \ell_{2}\right)_{0}$ and $M_{X}^{H}(v)_{0}$ are open subschemes of $\mathfrak{M}^{+}\left(v_{1}, v_{2}, \ell_{1}, \ell_{2}\right)$ and $M_{X}^{H}(v)$ respectively, and we have an isomorphism

$$
\begin{aligned}
\phi: \quad \mathfrak{M}^{+}\left(v_{1}, v_{2}, \ell_{1}, \ell_{2}\right)_{0} & \rightarrow M_{X}^{H}(v)_{0} \\
V^{\bullet} & \mapsto V^{\bullet}[k] .
\end{aligned}
$$

(2) If there is a complex $V^{\bullet} \in \mathfrak{M}^{+}\left(v_{1}, v_{2}, \ell_{1}, \ell_{2}\right)$ with $V^{\bullet}[k] \in \operatorname{Coh}(X)$ for some $k \in \mathbb{Z}$, then $\mathfrak{M}^{+}\left(v_{1}, v_{2}, \ell_{1}, \ell_{2}\right)_{0} \neq \emptyset$. 
Proof. (1) The openness of $\mathfrak{M}^{+}\left(v_{1}, v_{2}, \ell_{1}, \ell_{2}\right)_{0}$ is well-known (for example, see M7, Theorem 1.6 (1)]) and the existence of $\phi$ is the result of the universal property of $M_{X}^{H}(v)$. The openness of $M_{X}^{H}(v)_{0}$ and the existence of $\phi^{-1}$ follows from Proposition 4.8 (2).

(2) follows from Fact 2.17

4.3. Proof of Theorem 3.6. Before starting the proof, we prepare some lemmas.

Lemma 4.13. Let $E^{\bullet}$ be a bounded complex on $X$. Assume that $E^{\bullet}$ has two expressions

$$
E^{\bullet} \cong\left[E_{1} \rightarrow E_{2}\right] \cong\left[F_{1} \rightarrow F_{2}\right][-1],
$$

where $E_{1}, E_{2}, F_{1}, F_{2} \in \operatorname{Coh}(X)$. Then $E^{\bullet} \in \operatorname{Coh}(X)$ and $E:=H^{0}\left(E^{\bullet}\right)$ fits in the following two exact sequences in $\operatorname{Coh}(X)$.

$$
0 \rightarrow E_{1} \rightarrow E_{2} \rightarrow E \rightarrow 0, \quad 0 \rightarrow E \rightarrow F_{1} \rightarrow F_{2} \rightarrow 0 .
$$

Proof. We have two exact triangles:

$$
E_{1} \rightarrow E_{2} \rightarrow E^{\bullet} \rightarrow E_{1}[1], \quad E^{\bullet} \rightarrow F_{1} \rightarrow F_{2} \rightarrow E^{\bullet}[1] .
$$

By taking the cohomology groups of these triangles, we get the claims.

Lemma 4.14. Assume that a complex $E^{\bullet}$ on $X$ has two expressions

$$
E^{\bullet} \cong\left[E_{1} \stackrel{\phi}{\rightarrow} E_{2}\right] \cong\left[F_{2} \stackrel{\psi}{\rightarrow} F_{1}[2]\right][-1],
$$

where $E_{1}, E_{2}, F_{1}, F_{2}$ are semi-homogeneous sheaves and $\phi, \psi$ are non-trivial. Namely, we have an exact sequence in $\operatorname{Coh}(X)$ :

$$
0 \rightarrow F_{1} \rightarrow E_{1} \rightarrow E_{2} \rightarrow F_{2} \rightarrow 0 .
$$

Let $\Phi_{X \rightarrow Y}^{\mathbf{E}^{\vee}}$ be a FMT. Then the following holds.

(1) (a) If $\mu\left(F_{1}\right) \leq \mu\left(\left.\mathbf{E}\right|_{X \times\{y\}}\right)<\mu\left(E_{1}\right)$, then $\Phi_{X \rightarrow Y}^{\mathbf{E}^{\vee}}\left(E^{\bullet}\right) \in \operatorname{Coh}(Y)$.

(b) If $\mu\left(E_{2}\right) \leq \mu\left(\left.\mathbf{E}\right|_{X \times\{y\}}\right)<\mu\left(F_{2}\right)$, then $\Phi_{X \rightarrow Y}^{\mathbf{E}^{\vee}[1]}\left(E^{\bullet}\right) \in \operatorname{Coh}(Y)$.

(c) For the other cases, $\Phi_{X \rightarrow Y}^{\mathbf{E}^{\vee}[k]}\left(E^{\bullet}\right)$ is not a coherent sheaf on $Y$ for any $k$.

(2) (a) If $\mu\left(F_{1}\right)<\mu\left(\left.\mathbf{E}\right|_{X \times\{y\}}\right) \leq \mu\left(E_{1}\right)$, then $\mathcal{D}_{Y} \Phi_{X \rightarrow Y}^{\mathbf{E}^{\vee}}\left(E^{\bullet}\right) \in \operatorname{Coh}(Y)$.

(b) If $\mu\left(E_{2}\right)<\mu\left(\left.\mathbf{E}\right|_{X \times\{y\}}\right) \leq \mu\left(F_{2}\right)$, then $\mathcal{D}_{Y} \Phi_{X \rightarrow Y}^{\mathbf{E}^{\vee}[1]}\left(E^{\bullet}\right) \in \operatorname{Coh}(Y)$.

(c) For the other cases, $\mathcal{D}_{Y} \Phi_{X \rightarrow Y}^{\mathbf{E}^{\vee}[k]}\left(E^{\bullet}\right)$ is not a coherent sheaf on $Y$ for any $k$.

Proof. (1) We have an isomorphisms

$$
\Phi_{X \rightarrow Y}^{\mathbf{E}^{\vee}}\left(E^{\bullet}\right) \cong\left[\Phi_{X \rightarrow Y}^{\mathbf{E}^{\vee}}\left(E_{1}\right) \rightarrow \Phi_{X \rightarrow Y}^{\mathbf{E}^{\vee}}\left(E_{2}\right)\right] \cong\left[\Phi_{X \rightarrow Y}^{\mathbf{E}^{\vee}}\left(F_{2}\right) \rightarrow \Phi_{X \rightarrow Y}^{\mathbf{E}^{\vee}}\left(F_{1}\right)[2]\right][-1] .
$$

If $\mu\left(F_{1}\right) \leq \mu\left(\left.\mathbf{E}\right|_{X \times\{y\}}\right)<\mu\left(E_{1}\right)$, then

$$
\Phi_{X \rightarrow Y}^{\mathbf{E}^{\vee}}\left(F_{1}[2]\right), \Phi_{X \rightarrow Y}^{\mathbf{E}^{\vee}}\left(E_{1}\right), \Phi_{X \rightarrow Y}^{\mathbf{E}^{\vee}}\left(E_{2}\right), \Phi_{X \rightarrow Y}^{\mathbf{E}^{\vee}}\left(F_{2}\right) \in \operatorname{Coh}(Y) .
$$

Hence the claim (a) holds by Lemma 4.13 .

If $\mu\left(E_{2}\right) \leq \mu\left(\left.\mathbf{E}\right|_{X \times\{y\}}\right)<\mu\left(F_{2}\right)$, then

$$
\Phi_{X \rightarrow Y}^{\mathbf{E}^{\vee}}\left(F_{1}[2]\right), \Phi_{X \rightarrow Y}^{\mathbf{E}^{\vee}}\left(E_{1}[2]\right), \Phi_{X \rightarrow Y}^{\mathbf{E}^{\vee}}\left(E_{2}[2]\right), \Phi_{X \rightarrow Y}^{\mathbf{E}^{\vee}}\left(F_{2}\right) \in \operatorname{Coh}(Y) .
$$

Hence the claim (b) holds by Lemma 4.13 ,

For the proof of (c), we check the following three cases.

If $\mu\left(\left.\mathbf{E}\right|_{X \times\{y\}}\right)<\mu\left(F_{1}\right)$, then

$$
\Phi_{X \rightarrow Y}^{\mathbf{E}^{\vee}}\left(F_{1}\right), \Phi_{X \rightarrow Y}^{\mathbf{E}^{\vee}}\left(E_{1}\right), \Phi_{X \rightarrow Y}^{\mathbf{E}^{\vee}}\left(E_{2}\right), \Phi_{X \rightarrow Y}^{\mathbf{E}^{\vee}}\left(F_{2}\right) \in \operatorname{Coh}(Y) .
$$

Thus by (4.4) $\Phi_{X \rightarrow Y}^{\mathbf{E}^{\vee}[k]}\left(E^{\bullet}\right)$ cannot be a sheaf for any $k \in \mathbb{Z}$. 
If $\mu\left(E_{1}\right) \leq \mu\left(\left.\mathbf{E}\right|_{X \times\{y\}}\right)<\mu\left(E_{2}\right)$, then

$$
\Phi_{X \rightarrow Y}^{\mathbf{E}^{\vee}}\left(F_{1}[2]\right), \Phi_{X \rightarrow Y}^{\mathbf{E}^{\vee}}\left(E_{1}[2]\right), \Phi_{X \rightarrow Y}^{\mathbf{E}^{\vee}}\left(E_{2}\right), \Phi_{X \rightarrow Y}^{\mathbf{E}^{\vee}}\left(F_{2}\right) \in \operatorname{Coh}(Y) .
$$

By (4.4) $\Phi_{X \rightarrow Y}^{\mathbf{E}^{\vee}[k]}\left(E^{\bullet}\right)$ cannot be a sheaf for any $k \in \mathbb{Z}$.

If $\mu\left(F_{2}\right) \leq \mu\left(\left.\mathbf{E}\right|_{X \times\{y\}}\right)$, then

$$
\Phi_{X \rightarrow Y}^{\mathbf{E}^{\vee}}\left(F_{1}[2]\right), \Phi_{X \rightarrow Y}^{\mathbf{E}^{\vee}}\left(E_{1}[2]\right), \Phi_{X \rightarrow Y}^{\mathbf{E}^{\vee}}\left(E_{2}[2]\right), \Phi_{X \rightarrow Y}^{\mathbf{E}^{\vee}}\left(F_{2}[2]\right) \in \operatorname{Coh}(Y) .
$$

By (4.4) $\Phi_{X \rightarrow Y}^{\mathbf{E}^{\vee}[k]}\left(E^{\bullet}\right)$ cannot be a sheaf for any $k \in \mathbb{Z}$.

(2) We have an isomorphisms

$$
\Phi_{X \rightarrow Y}^{\mathbf{E}^{\vee}}\left(E^{\bullet}\right)^{\vee} \cong\left[\Phi_{X \rightarrow Y}^{\mathbf{E}^{\vee}}\left(E_{2}\right)^{\vee} \rightarrow \Phi_{X \rightarrow Y}^{\mathbf{E}^{\vee}}\left(E_{1}\right)^{\vee}\right][-1] \cong\left[\Phi_{X \rightarrow Y}^{\mathbf{E}^{\vee}}\left(F_{1}[2]\right)^{\vee} \rightarrow \Phi_{X \rightarrow Y}^{\mathbf{E}^{\vee}}\left(F_{2}\right)^{\vee}\right] .
$$

If $\mu\left(F_{1}\right)<\mu\left(\left.\mathbf{E}\right|_{X \times\{y\}}\right) \leq \mu\left(E_{1}\right)$, then

$$
\Phi_{X \rightarrow Y}^{\mathbf{E}^{\vee}}\left(E_{1}\right)^{\vee}, \Phi_{X \rightarrow Y}^{\mathbf{E}^{\vee}}\left(E_{2}\right)^{\vee}, \Phi_{X \rightarrow Y}^{\mathbf{E}^{\vee}}\left(F_{1}\right)^{\vee}, \Phi_{X \rightarrow Y}^{\mathbf{E}^{\vee}}\left(F_{1}[2]\right)^{\vee} \in \operatorname{Coh}(Y) .
$$

Hence the claim (a) holds by Lemma 4.13. The other claims are verified similarly as (1).

Now we can show Theorem 3.6 .

Proof of Theorem [3.6. The uniqueness statements follow from Proposition 3.3. In the following we show the existence statements.

(1) Assume that the numerical equation of $v$ has two solutions $\left(v_{1}, v_{2}, \ell_{1}, \ell_{2}\right)$ and $\left(v_{1}^{\prime}, v_{2}^{\prime}, \ell_{1}^{\prime}, \ell_{2}^{\prime}\right)$. By Proposition 4.7, we have two families of simple complexes $\mathcal{V}^{\bullet}=\left[\mathcal{V}^{-1} \rightarrow \mathcal{V}^{0}\right]$ and $\mathcal{V}^{\bullet}=$ $\left[\mathcal{V}^{\prime-1} \rightarrow \mathcal{V}^{\prime 0}\right]$ associated to the two numerical solutions $\left(v_{1}, v_{2}, \ell_{1}, \ell_{2}\right)$ and $\left(v_{1}^{\prime}, v_{2}^{\prime}, \ell_{1}^{\prime}, \ell_{2}^{\prime}\right)$. We set $S:=\mathfrak{M}^{+}\left(v_{1}, v_{2}, \ell_{1}, \ell_{2}\right)$ and $S^{\prime}:=\mathfrak{M}^{+}\left(v_{1}^{\prime}, v_{2}^{\prime}, \ell_{1}^{\prime}, \ell_{2}^{\prime}\right)$.

(a) We first assume that $\mathcal{V}_{s}^{\bullet}$ and $\mathcal{V}_{s^{\prime}}^{\prime}$ are sheaves up to shift for general $s \in S$ and $s^{\prime} \in S^{\prime}$. By Proposition 4.12, there are non-empty open subschemes $S_{0} \subset S$ and $S_{0}^{\prime} \subset S^{\prime}$, and we have open immersions $f: S_{0} \rightarrow M_{X}^{H}(v)$ and $f^{\prime}: S_{0}^{\prime} \rightarrow M_{X}^{H}(v)$. Since $M_{X}^{H}(v)$ is irreducible (Fact 2.3), $f\left(S_{0}\right) \cap f^{\prime}\left(S_{0}^{\prime}\right) \neq \emptyset$, which implies that a general member $E \in M_{X}^{H}(v)$ has two semi-homogeneous presentations. By the uniqueness of the presentations (Proposition 3.3), one of these two presentations must be a kernel presentation and the other should be a cokernel presentation. Thus we obtain the result.

(b) Next we assume that $\mathcal{V}_{s}^{\bullet}$ is not a sheaf for all $s \in S$. Then Fact 2.15 implies that there is a numerical solution $\left(u_{1}, u_{2}, k_{1}, k_{2}\right)$ such that for a general $s \in S, H^{-1}\left(\mathcal{V}_{s}^{\bullet}\right)$ and $H^{0}\left(\mathcal{V}_{s}^{\bullet}\right)$ are semihomogeneous sheaves with $v\left(H^{-1}\left(\mathcal{V}_{s}^{\bullet}\right)\right)=k_{1} u_{1}$ and $v\left(H^{0}\left(\mathcal{V}_{s}^{\bullet}\right)\right)=k_{2} u_{2}$ respectively. Applying Fact 2.17 to the family of complexes $\mathcal{V}^{\bullet}$ successively, we find an equivalence $\mathcal{F}: \mathbf{D}(X) \rightarrow \mathbf{D}(X)$ or $\mathbf{D}(X) \rightarrow \mathbf{D}(X)_{\text {op }}$ such that $\mathcal{F}\left(\mathcal{V}_{s}^{\bullet}\right) \cong G[k]$, where $G$ is a stable sheaf, $k \in \mathbb{Z}$ and $v(G[k])=v$. Then by the proof of Lemma 4.14, we have the kernel and the cokernel presentation of $G$.

(2) Assume that the numerical equation of $v$ has a unique solution $\left(v_{1}, v_{2}, \ell_{1}, \ell_{2}\right)$. We have a complex $\mathcal{V}^{\bullet}=\left[\mathcal{V}^{-1} \rightarrow \mathcal{V}^{0}\right]$ associated to this unique solution. This is a semi-homogeneous presentation, since otherwise we would have another numerical solution by Fact 2.15.

\section{Applications of the Semi-homogeneous presentation}

In this section we give some applications of the semi-homogeneous presentations. In particular Mukai's Conjecture 5.1 is proved, as mentioned in the introduction. We fix an abelian surface $X$ and suppose $\operatorname{NS}(X)=\mathbb{Z} H$ in order to use Theorem 3.6 . 
5.1. An old conjecture of Mukai. In this subsection we give an answer to the following Conjecture [5.1, originally proposed by Mukai [M3, Conjecture 1'].

Conjecture 5.1. Let $X$ be an abelian surface with $\operatorname{NS}(X)=\mathbb{Z} H$ and $v$ be a Mukai vector with $\ell:=\left\langle v^{2}\right\rangle / 2>0$. Suppose that $v$ has at least one solution of the numerical equation (3.2).

(1) Among the numerical solutions for $v$, take the solution $\left(v_{1}, v_{2}, \ell_{1}, \ell_{2}\right)$ such that $\operatorname{rk}\left(v_{i}\right)$ is minimum, where the index $i \in\{1,2\}$ is determined by $\ell_{i}=\ell$. Then for a general member $\left[E^{-1} \stackrel{f}{\rightarrow} E^{0}\right]$ of $\mathfrak{M}^{+}\left(v_{1}, v_{2}, \ell_{1}, \ell_{2}\right), f$ is either surjective or injective.

(2) In the situation of (1), the kernel or cokernel of $f$ is stable.

(3) A general member of $M_{X}^{H}(v)$ has a semi-homogeneous presentation corresponding to the numerical solution $\left(v_{1}, v_{2}, \ell_{1}, \ell_{2}\right)$ of $v$ such that $\operatorname{rk}\left(v_{i}\right)$ is minimum, where the index $i \in\{1,2\}$ is determined by $\ell_{i}=\ell$.

Theorem 5.2. Conjecture 5.1 is true.

Proof. (1) If $v=e^{d H}(1,0,-\ell)$ or $v=e^{d H}(\ell, 0,-1)$ with $d \in \mathbb{Z}$, then we have obvious solutions $v=-\left(\ell e^{d H}(0,0,1)-e^{d H}(1,0,0)\right)$ or $v=\ell e^{d H}(1,0,0)-e^{d H}(0,0,1)$, and the claim holds.

Hence we may assume that $v \neq e^{d H}(1,0,-\ell)$ nor $v \neq e^{d H}(\ell, 0,-1)$. Let $\left(v_{1}, v_{2}, \ell_{1}, \ell_{2}\right)$ be any numerical solution of $v$. Let $\mathcal{V}^{\bullet}: \mathcal{V}^{-1} \rightarrow \mathcal{V}^{0}$ be the family of simple complexes on $X$ in Proposition 4.7 (2). Namely, we have $\mathcal{V}_{s}^{-1} \in M_{X}^{H}\left(\ell_{1} v_{1}\right)^{s s}$ and $\mathcal{V}_{s}^{0} \in M_{X}^{H}\left(\ell_{2} v_{2}\right)^{s s}$ for $s \in$ $\mathfrak{S}:=\mathfrak{M}^{+}\left(v_{1}, v_{2}, \ell_{1}, \ell_{2}\right)$.

In order to prove the statement, it is sufficient to prove the following: if $\mathcal{V}_{s}^{\bullet}[k], k=-1,0$ is not a sheaf for all $s \in \mathfrak{S}$, then there is another numerical solution $\left(v_{1}^{\prime}, v_{2}^{\prime}, \ell_{1}^{\prime}, \ell_{2}^{\prime}\right)$ of $v$ such that $\operatorname{rk}\left(v_{i}^{\prime}\right)<\min \left\{\operatorname{rk}\left(v_{1}\right), \operatorname{rk}\left(v_{2}\right)\right\}$ for $i=1,2$.

Assume that $\mathcal{V}_{s}^{\bullet}[k], k=-1,0$ is not a sheaf for all $s \in \mathfrak{S}$. By Fact 2.12, there is another numerical solution $\left(v_{1}^{\prime}, v_{2}^{\prime}, \ell_{1}^{\prime}, \ell_{2}^{\prime}\right)$ such that $H^{-1}\left(\mathcal{V}_{s}^{\bullet}\right) \in M_{X}^{H}\left(\ell_{1}^{\prime} v_{1}^{\prime}\right)^{s s}$ and $H^{0}\left(\mathcal{V}_{s}^{\bullet}\right) \in M_{X}^{H}\left(\ell_{2}^{\prime} v_{2}^{\prime}\right)^{s s}$ for a general $s \in \mathfrak{S}$. By Lemma 4.5, $\Phi_{X \rightarrow Y_{1}}^{\mathbf{E}_{1}^{\vee}[1]}\left(\mathcal{V}^{\bullet}\right)$ is a family of simple complex with Mukai vector $\left(\ell_{2}, 0,-\ell_{1}\right)$. Since $\mathcal{V}_{s}^{\bullet} \cong\left[H^{0}\left(\mathcal{V}_{s}^{\bullet}\right) \rightarrow H^{-1}\left(\mathcal{V}_{s}^{\bullet}\right)[2]\right][-1]$, we have

$$
\begin{aligned}
\Phi_{X \rightarrow Y_{1}}^{\mathbf{E}_{1}^{\vee}}\left(\mathcal{V}_{s}^{\bullet}\right) & \cong\left[\Phi_{X \rightarrow Y_{1}}^{\mathbf{E}_{1}^{\vee}}\left(H^{0}\left(\mathcal{V}_{s}^{\bullet}\right)\right) \rightarrow \Phi_{X \rightarrow Y_{1}}^{\mathbf{E}_{1}^{\vee}}\left(H^{-1}\left(\mathcal{V}_{s}^{\bullet}\right)\right)[2]\right][-1] \\
& \cong\left[\Phi_{X \rightarrow Y_{1}}^{\mathbf{E}_{1}^{\vee}}\left(H^{0}\left(\mathcal{V}_{s}^{\bullet}\right)\right) \rightarrow \Phi_{X \rightarrow Y_{1}}^{\mathbf{E}_{1}^{\vee}[2]}\left(H^{-1}\left(\mathcal{V}_{s}^{\bullet}\right)\right)\right][-1] .
\end{aligned}
$$

Since $\mu\left(v_{1}^{\prime}\right)<\mu\left(v_{1}\right)<\mu\left(v_{2}^{\prime}\right), \Phi_{X \rightarrow Y_{1}}^{\mathbf{E}_{1}^{\vee}[2]}\left(H^{-1}\left(\mathcal{V}_{s}^{\bullet}\right)\right)$ and $\Phi_{X \rightarrow Y_{1}}^{\mathbf{E}_{1}^{\vee}}\left(H^{0}\left(\mathcal{V}_{s}^{\bullet}\right)\right)$ are semi-homogeneous vector bundles with Mukai vector $\ell_{1}^{\prime} u_{1}$ and $\ell_{2}^{\prime} u_{2}$ respectively. Here we defined $u_{1}:=\Phi_{X \rightarrow Y_{1}}^{\mathbf{E}_{1}^{\vee}}\left(v_{1}^{\prime}\right)$ and $u_{2}:=\Phi_{X \rightarrow Y_{1}}^{\mathbf{E}_{1}^{\vee}}\left(v_{2}^{\prime}\right)$.

Now we show the inequality $\operatorname{rk}\left(v_{1}\right)-\operatorname{rk}\left(v_{i}^{\prime}\right)>0$. We note that $0=\mu\left(\Phi_{X \rightarrow Y_{1}}^{\mathbf{E}_{1}^{\vee}}\left(v_{2}\right)\right)<\mu\left(u_{2}\right) \leq$ $\mu\left(\left.\mathbf{E}_{1}\right|_{\{x\} \times Y_{1}} ^{\vee}\right)<\mu\left(u_{1}\right)$. If $u_{2}=v\left(\left.\mathbf{E}_{1}\right|_{\{x\} \times Y_{1}} ^{\vee}\right)$ and $\operatorname{rk}\left(u_{2}\right)=\operatorname{rk}\left(v_{1}\right)=1$, then $\operatorname{rk}\left(v_{1}^{\prime}\right)=1$ and $v_{2}^{\prime}=(0,0,1)$, which means that $v=e^{d H}(1,0,-\ell)$, or $e^{d H}(\ell, 0,-1)$, contradicting the assumption. Therefore $u_{2} \neq v\left(\left.\mathbf{E}_{1}\right|_{\{x\} \times Y_{1}} ^{\vee}\right)$ or $\operatorname{rk}\left(u_{2}\right) \neq 1$. Since

$$
\begin{aligned}
\operatorname{rk}\left(v_{1}\right)-\operatorname{rk}\left(v_{i}^{\prime}\right) & =\operatorname{rk}\left(\Phi_{Y_{1} \rightarrow X}^{\mathbf{E}_{1}[2]}((0,0,1))\right)-\operatorname{rk}\left(\Phi_{Y_{1} \rightarrow X}^{\mathbf{E}_{1}[2]}\left(u_{i}\right)\right) \\
& =-\left\langle v\left(\left.\mathbf{E}_{1}\right|_{\{x\} \times Y_{1}} ^{\vee}\right),(0,0,1)\right\rangle+\left\langle v\left(\left.\mathbf{E}_{1}\right|_{\{x\} \times Y_{1}} ^{\vee}\right), u_{i}\right\rangle,
\end{aligned}
$$

the claim follows from Lemma 5.3 below.

Next we deal with the inequality $\operatorname{rk}\left(v_{2}\right)-\operatorname{rk}\left(v_{i}^{\prime}\right)>0$. By Lemma 4.5, $\Phi_{X \rightarrow Y_{2}}^{\mathbf{E}_{2}^{\vee}[1]}\left(\mathcal{V}^{\bullet}\right)$ is a family of stable sheaves with Mukai vector $\left(\ell_{1}, 0,-\ell_{2}\right)$. Since $\mathcal{V}_{s}^{\bullet} \cong\left[H^{0}\left(\mathcal{V}_{s}^{\bullet}\right) \rightarrow H^{-1}\left(\mathcal{V}_{s}^{\bullet}\right)[2]\right][-1]$, we have

$$
\Phi_{X \rightarrow Y_{2}}^{\mathbf{E}_{2}^{\vee}[1]}\left(\mathcal{V}_{s}^{\bullet}\right) \cong\left[\Phi_{X \rightarrow Y_{2}}^{\mathbf{E}_{2}^{\vee}[1]}\left(H^{0}\left(\mathcal{V}_{s}^{\bullet}\right)\right)[-1] \rightarrow \Phi_{X \rightarrow Y_{2}}^{\mathbf{E}_{2}^{\vee}[1]}\left(H^{-1}\left(\mathcal{V}_{s}^{\bullet}\right)\right)[1]\right]
$$




$$
\cong\left[\Phi_{X \rightarrow Y_{2}}^{\mathbf{E}_{2}^{\vee}}\left(H^{0}\left(\mathcal{V}_{s}^{\bullet}\right)\right) \rightarrow \Phi_{X \rightarrow Y_{2}}^{\mathbf{E}_{2}^{\vee}[2]}\left(H^{-1}\left(\mathcal{V}_{s}^{\bullet}\right)\right)\right]
$$

Since $\mu\left(v_{1}^{\prime}\right)<\mu\left(v_{2}\right)<\mu\left(v_{2}^{\prime}\right), \Phi_{X \rightarrow Y_{2}}^{\mathbf{E}_{2}^{\vee}[2]}\left(H^{-1}\left(\mathcal{V}_{s}^{\bullet}\right)\right)$ and $\Phi_{X \rightarrow Y_{2}}^{\mathbf{E}_{2}^{\vee}}\left(H^{0}\left(\mathcal{V}_{s}^{\bullet}\right)\right)$ are semi-homogeneous vector bundles with Mukai vector $\ell_{1}^{\prime} u_{1}^{\prime}$ and $\ell_{2}^{\prime} u_{2}^{\prime}$ respectively, where $u_{1}^{\prime}:=\Phi_{X \rightarrow Y_{2}}^{\mathbf{E}_{2}^{\vee}}\left(v_{1}^{\prime}\right)$ and $u_{2}^{\prime}:=$ $\Phi_{X \rightarrow Y_{2}}^{\mathbf{E}_{2}^{\vee}}\left(v_{2}^{\prime}\right)$. Note that $\mu\left(u_{2}^{\prime}\right) \leq \mu\left(\left.\mathbf{E}_{2}\right|_{\{x\} \times Y_{2}} ^{\vee}\right)<\mu\left(u_{1}^{\prime}\right)<0$. Then we can prove the inequality $\operatorname{rk}\left(v_{2}\right)-\operatorname{rk}\left(v_{i}^{\prime}\right)>0$ using Lemma 5.3 below and a similar argument as above.

$(2),(3)$ These are the consequences of (1), Proposition 4.12 and the irreducibility of $M_{X}^{H}(v)$ (Fact 2.3).

Lemma 5.3. Let $Y$ be an abelian surface with $\mathrm{NS}(Y)=\mathbb{Z} H^{\prime}$ and $w_{i}:=\left(r_{i}, d_{i} H^{\prime}, d_{i}^{2}\left(H^{\prime 2}\right) /\left(2 r_{i}\right)\right)$ $(i=1,2,3)$ be primitive and isotropic Mukai vectors on $Y$ such that $r_{i}>0,\left\langle w_{1}, w_{3}\right\rangle=-1$, $\mu\left(w_{1}\right) \leq \mu\left(w_{2}\right) \leq \mu\left(w_{3}\right)$ and $d_{1} d_{3}>0$. Then we have $-\left\langle w_{2},(0,0,1)\right\rangle+\left\langle w_{2}, w_{i}\right\rangle \geq 0$. If the equality holds, then $\operatorname{rk} w_{2}=1$ and $w_{j}=w_{2}$, where $\{i, j\}=\{1,3\}$.

Proof. For $i, j$ with $\{i, j\}=\{1,3\}$,

$$
\begin{aligned}
-\left\langle w_{2},(0,0,1)\right\rangle+\left\langle w_{2}, w_{i}\right\rangle & =r_{2}-r_{2} r_{i}\left(\frac{d_{2}}{r_{2}}-\frac{d_{i}}{r_{i}}\right)^{2} \frac{\left(H^{\prime 2}\right)}{2} \\
& \geq r_{2}-r_{2} r_{i}\left(\frac{d_{j}}{r_{j}}-\frac{d_{i}}{r_{i}}\right)^{2} \frac{\left(H^{\prime 2}\right)}{2}=r_{2}+\frac{r_{2}}{r_{j}}\left\langle w_{i}, w_{j}\right\rangle=r_{2}\left(1-\frac{1}{r_{j}}\right) \geq 0 .
\end{aligned}
$$

If the equality holds, then $\mu\left(w_{2}\right)=\mu\left(w_{j}\right)$ and $r_{j}=1$. Hence $w_{j}=w_{2}$ and $\operatorname{rk}\left(w_{2}\right)=1$.

5.2. Sheaf-preserving Fourier-Mukai transforms. Since FMTs are defined in the derived category, we cannot expect that a sheaf is transformed into a sheaf in general. In Lemma 4.13 of the previous section, we have already encountered such a phenomenon. Similar statements are dealt in the next proposition.

Proposition 5.4. Suppose that a coherent sheaf $E$ has both kernel and cokernel presentations.

$$
0 \rightarrow E_{1} \rightarrow E_{2} \rightarrow E \rightarrow 0, \quad 0 \rightarrow E \rightarrow F_{1} \rightarrow F_{2} \rightarrow 0 .
$$

Let $\mathbf{E}$ be a universal family of simple semi-homogeneous sheaves. Let $\mu:=\mu\left(\mathbf{E}_{y}\right), y \in Y$. The image of $E$ by $\Phi:=\Phi_{X \rightarrow Y}^{\mathbf{E}^{\vee}}$ is classified as follows.

\begin{tabular}{r|ll} 
range of slope & image of $E$ & $\begin{array}{l}\text { non-vanishing } \\
\text { cohomology sheaf }\end{array}$ \\
\hline$\mu<\mu\left(E_{1}\right)$ & genuine sheaf & $\Phi^{0}(E)$ \\
$\mu\left(E_{1}\right) \leq \mu<\mu\left(E_{2}\right)$ & 2-term cpx. & $\Phi^{0}(E), \Phi^{1}(E)$ \\
$\mu\left(E_{2}\right) \leq \mu<\mu\left(F_{1}\right)$ & genuine sheaf & $\Phi^{1}(E)$ \\
$\mu\left(F_{1}\right) \leq \mu<\mu\left(F_{2}\right)$ & 2-term cpx. & $\Phi^{1}(E), \Phi^{2}(E)$ \\
$\mu\left(F_{2}\right) \leq \mu$ & genuine sheaf & $\Phi^{2}(E)$
\end{tabular}

Proof. This is the consequence of WIT for semi-homogeneous sheaves (Proposition 2.14). Since the proof is similar to that of Lemma 4.14, we omit the detail.

Example 5.5. Suppose $\operatorname{NS}(X)=\mathbb{Z} H$ and put $n:=\left(H^{2}\right) / 2 \in \mathbb{Z}_{>0}$. We can give the condition when a sheaf is converted into a complex under the FMT $\Phi:=\Phi_{X \rightarrow \widehat{X}}^{\mathbf{P}}$, where $\mathbf{P}$ is the Poincaré bundle of $X$. Assume that the Mukai vector $v$ with $\left\langle v^{2}\right\rangle>0$ has a numerical solution. Then for a general member $E \in M_{X}^{H}(v)$, the image of $E$ under $\Phi$ is a two-term complex only if $v(E)$ can be written by one of the following four forms.
(a) $\left(\ell d^{2} n-1, \ell d H, \ell\right)$
with $d>0$,
(c) $\left(\ell-d^{2} n,-d H,-1\right)$
with $d>0$,
(b) $\left(d^{2} n-\ell, d H, 1\right)$ with $d>0$,
(d) $(1,0,-\ell)$. 
Proof. By Theorem 3.6, we can assume that $E$ has a semi-homogeneous presentation. We divide the argument into two cases according to the kind of the presentation.

(1) Assume that $E$ has a cokernel presentation

$$
0 \rightarrow E_{1} \rightarrow E_{2} \rightarrow E \rightarrow 0
$$

and that the image of $E$ by $\Phi$ is not a sheaf under any shift.

(i) First consider the case

$$
v\left(E_{1}\right)=v_{1}=\left(r_{1}, d_{1} H, a_{1}\right), \quad v\left(E_{2}\right)=\ell v_{2}=\ell\left(r_{2}, d_{2} H, a_{2}\right),
$$

where $v_{1}$ and $v_{2}$ are primitive. Then

$$
\begin{aligned}
& \left\langle v_{1}, v_{2}\right\rangle=-1 \Longleftrightarrow 2 d_{1} d_{2} n=r_{1} a_{2}+r_{2} a_{1}-1, \\
& \left\langle v_{1}^{2}\right\rangle=0 \Longleftrightarrow d_{1}^{2} n=r_{1} a_{1}, \quad\left\langle v_{2}^{2}\right\rangle=0 \Longleftrightarrow d_{2}^{2} n=r_{2} a_{2} .
\end{aligned}
$$

By the result of Proposition 5.4 and the equality $\mu\left(\mathbf{P}_{y}\right)=0$, we need to determine the condition for $\mu\left(E_{1}\right) \leq 0<\mu\left(E_{2}\right)$. If $\mu\left(E_{1}\right) \neq 0$, then we have

$$
r_{1} a_{2}+r_{2} a_{1}-1<0, \quad r_{1} a_{1}>0 \text { and } \quad r_{2} a_{2}>0 .
$$

These inequities have no solution, so that we may assume $\mu\left(E_{1}\right)=0<\mu\left(E_{2}\right)$. This condition yields

$$
r_{1}>0, \quad d_{1}=0, \quad d_{2} \geq 0 \quad \text { and } \quad r_{2} \geq 0 .
$$

These ineqalities have only one solution:

$$
v_{1}=(1,0,0), \quad v_{2}=\left(d^{2} n, d H, 1\right), \quad d \geq 0 .
$$

From the condition $\operatorname{rk}(E)>0$ we have $d>0$. Thus we obtained the first solution (a).

(ii) Next consider the case

$$
v\left(E_{1}\right)=\ell v_{1}=\ell\left(r_{1}, d_{1} H, a_{1}\right), \quad v\left(E_{2}\right)=v_{2}=\left(r_{2}, d_{2} H, a_{2}\right) .
$$

By the result of Proposition 5.4, we need to determine the condition for $\mu\left(E_{1}\right) \leq 0<\mu\left(E_{2}\right)$. As in the first case, $\mu\left(E_{1}\right)<0<\mu\left(E_{2}\right)<\infty$ has no solution. Then we have to consider the case $\mu\left(E_{1}\right)=0<\mu\left(E_{2}\right)$. This case has the next solution

$$
v_{1}=(1,0,0), \quad v_{2}=\left(d^{2} n, d H, 1\right) \quad d \geq 0,
$$

and we can exclude the case $d=0$ by the condition $\operatorname{rk}(E)>0$. Hence the second solution (b) is obtained.

(2) Assume that $E$ has a kernel presentation

$$
0 \rightarrow E \rightarrow E_{2} \rightarrow E_{1} \rightarrow 0
$$

and the image of $E$ is a two-term complex. We can put $v\left(E_{1}\right)=\ell_{1} v_{1}$ and $v\left(E_{2}\right)=\ell_{2} v_{2}$ with $\left(\ell_{1}, \ell_{2}\right)=(1, \ell)$ or $(\ell, 1)$. The condition we have to consider is $\mu\left(E_{1}\right) \leq 0<\mu\left(E_{2}\right)$. Then a simple calculation as in (1) shows that the solutions are

$$
v_{1}=\left(d^{2} n, d H, 1\right), \quad v_{2}=(1,0,0), \quad d \geq 0 .
$$

Considering the condition $\operatorname{rk}(E)>0$, we obtain the third solution (c) and the last one (d).

Remark 5.6. In the cases (a) and (b), one may easily find that $\mathcal{D}_{\widehat{X}} \circ \Phi$ induces a birational map $M_{X}^{H}(v) \cdots \rightarrow X \times \operatorname{Hilb}^{\ell}(\widehat{X})$. Thus we recover the result of [Y3, Theorem 3.14. (1)].

As a consequence of Theorem 3.6 and Proposition 5.4, we have the next proposition. 
Proposition 5.7. Suppose that $\operatorname{NS}(X)=\mathbb{Z} H,\left(H^{2}\right)=2 n$ and that $v$ is a primitive Mukai vector. Then for any universal family $\mathbf{E}$ of simple semi-homogeneous sheaves over $Y=M_{X}^{H}\left(v^{\prime}\right)$ with $v^{\prime}=\left(r^{\prime}, d^{\prime} H, a^{\prime}\right)$ satisfying

$$
\frac{d^{\prime}}{r^{\prime}}<\frac{d}{r}-\frac{\sqrt{r+1}}{r} \sqrt{\frac{\ell}{n}}
$$

or

$$
\frac{d^{\prime}}{r^{\prime}}>\frac{d}{r}+\frac{\sqrt{r+1}}{r} \sqrt{\frac{\ell}{n}}
$$

the image $\Phi_{X \rightarrow Y}^{\mathbf{E}}(E)$ of a general member $E \in M_{X}^{H}(v)$ is a stable sheaf up to shift.

Proof. We only show the proof for the case (5.1), since the other case can be shown quite similarly.

By Fact 2.15, we may assume that $v$ has a numerical solution. By Theorem [3.6, we can assume that $E$ has a semi-homogeneous presentation

$$
\begin{aligned}
& \text { (1) } 0 \rightarrow E \rightarrow E_{1} \rightarrow E_{2} \rightarrow 0 \text {, or } \\
& \text { (2) } 0 \rightarrow E_{2} \rightarrow E_{1} \rightarrow E \rightarrow 0, \quad \text { or } \\
& \text { (3) } 0 \rightarrow E \rightarrow E_{2} \rightarrow E_{1} \rightarrow 0, \quad \text { or } \\
& \text { (4) } 0 \rightarrow E_{1} \rightarrow E_{2} \rightarrow E \rightarrow 0
\end{aligned}
$$

with $v\left(E_{1}\right)=\ell v_{1}, v\left(E_{2}\right)=v_{2}$. By Lemma 6.14 proved later, we can set

$$
\begin{aligned}
& v_{1}=\left(p_{1}^{2} r_{1}, p_{1} q_{1} H, q_{1}^{2} r_{2}\right), \quad v_{2}=\left(p_{2}^{2} r_{2}, p_{2} q_{2} H, q_{2}^{2} r_{1}\right), \\
& r_{1} r_{2}=n, \quad p_{1}>0, \quad p_{1} q_{2} r_{1}-p_{2} q_{1} r_{2}=1 .
\end{aligned}
$$

The cases (1) and (3) follows from Proposition 5.4, since for each case we have

$$
\mu\left(v^{\prime}\right)<\mu(v)<\mu\left(v_{1}\right)<\mu\left(v_{2}\right), \quad \mu\left(v^{\prime}\right)<\mu(v)<\mu\left(v_{2}\right)<\mu\left(v_{1}\right) .
$$

For the case (2), we estimate the value of $\mu(v)-\mu\left(v_{2}\right)$. From $v=\ell v_{1}-v_{2}$ we have $r=$ $\ell p_{1}^{2} r_{1}-p_{2}^{2} r_{2}$ and $d=\ell p_{1} q_{1}-p_{2} q_{2}$. Then we have

$$
\mu(v)-\mu\left(v_{2}\right)=\frac{\ell p_{1} q_{1}-p_{2} q_{2}}{\ell p_{1}^{2} r_{1}-p_{2}^{2} r_{2}}-\frac{q_{2}}{p_{2} r_{2}}=\frac{-\ell}{r} \frac{p_{1}}{p_{2} r_{2}} .
$$

Notice that $p_{2}<0$ holds, since

$$
\begin{aligned}
\mu\left(v_{2}\right)<\mu\left(v_{1}\right) & \Longleftrightarrow \frac{q_{2}}{p_{2} r_{2}}<\frac{q_{1}}{p_{1} r_{1}} \Longleftrightarrow 0<\frac{q_{1}}{p_{1} r_{1}}-\frac{q_{2}}{p_{2} r_{2}}=\frac{p_{2} q_{1} r_{2}-p_{1} q_{2} r_{1}}{p_{1} p_{2} r_{1} r_{2}} \\
& \Longleftrightarrow 0<-\frac{1}{p_{1} p_{2} n} \Longleftrightarrow p_{2}<0 .
\end{aligned}
$$

We also find from $r=\ell p_{1}^{2} r_{1}-p_{2}^{2} r_{2}$ that $p_{1}^{2} / p_{2}^{2} r_{2}^{2}=\left(1+r / p_{2}^{2} r_{2}\right) / \ell n$, which leads to $p_{1}^{2} / p_{2}^{2} r_{2}^{2} \leq$ $(r+1) / \ell n$. The equality holds if and only if $p_{2}^{2} r_{2}=1$. Hence we have

$$
\mu(v)-\mu\left(v_{2}\right) \leq \frac{\sqrt{r+1}}{r} \sqrt{\frac{\ell}{n}} .
$$

Therefore the conclusion follows from Proposition 5.4, since the assumption (5.1) means

$$
\mu\left(v^{\prime}\right)<\mu(v)-\frac{\sqrt{r+1}}{r} \sqrt{\frac{\ell}{n}} \leq \mu\left(v_{2}\right)
$$

for any choice of $v_{1}$ and $v_{2}$.

For the case (4), we have $r=p_{2}^{2} r_{2}-\ell p_{1}^{2} r_{1}$ and $d=p_{2} q_{2}-\ell p_{1} q_{1}$. Then

$$
\mu(v)-\mu\left(v_{1}\right)=\frac{p_{2} q_{2}-\ell p_{1} q_{1}}{p_{2}^{2} r_{2}-\ell p_{1}^{2} r_{1}}-\frac{q_{1}}{p_{1} r_{1}}=\frac{1}{r} \frac{p_{2}}{p_{1} r_{1}} .
$$


In this case we have $p_{2}>0$ and $p_{2}^{2} / p_{1}^{2} r_{1}^{2}=\left(\ell+r / p_{1}^{2} r_{1}\right) / n$, which means that $(r+\ell) p_{2}^{2} / n p_{1}^{2} r_{1}^{2}>$ $\ell / n$. Hence we have

$$
\mu(v)-\mu\left(v_{1}\right) \leq \frac{1}{r} \sqrt{\frac{\ell+r}{n}} .
$$

Since $\sqrt{\ell(r+1) / n} \geq \sqrt{(\ell+r) / n}$, the assumption (5.1) means that

$$
\mu\left(v^{\prime}\right)<\mu(v)-\frac{1}{r} \sqrt{\frac{\ell+r}{n}} \leq \mu-\left(\mu-\mu_{1}\right)=\mu_{1} .
$$

Therefore the conclusion holds by Proposition 5.4 and Fact 2.17 (1).

\section{Matrix Description of COHOMOlogical FMT}

This section is devoted to the description of cohomological FMT via quadratic matrices. The original idea is due to Mukai [M2, M3]. We fix an abelian surface $X$ with $\mathrm{NS}(X)=\mathbb{Z} H$ and set $\left(H^{2}\right)=2 n$.

Our study can be summarized in the next diagram.

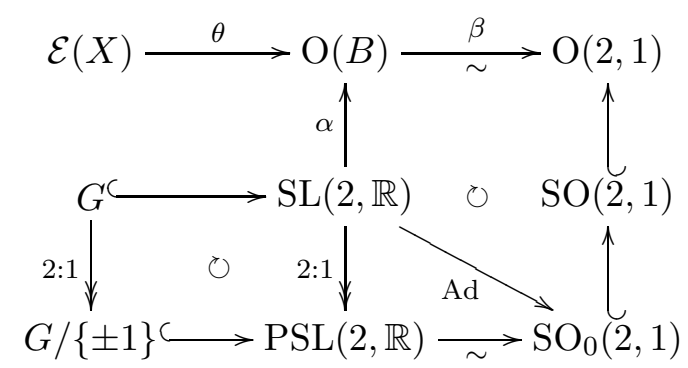

Here $\mathrm{O}(2,1), \mathrm{SO}(2,1), \mathrm{SL}(2, \mathbb{R}), \operatorname{PSL}(2, \mathbb{R})$ are the familiar real Lie groups, regarded as subgroups of the group of invertible $3 \times 3$ matrices $\mathrm{GL}(3, \mathbb{R})$. $\mathrm{SO}(2,1)$ has two connected components and $\mathrm{SO}_{0}(2,1)$ denotes the component of $\mathrm{SO}(2,1)$ including the identity matrix. We are interested in the set

$$
\mathcal{E}(X):=\bigcup_{Y \in \mathrm{FM}(X)} \operatorname{Eq}_{0}(\mathbf{D}(Y), \mathbf{D}(X))
$$

where $\mathrm{Eq}_{0}(\mathbf{D}(Y), \mathbf{D}(X))$ is a subset of $\mathrm{Eq}(\mathbf{D}(Y), \mathbf{D}(X))$ defined in Definition 6.15)(1). Although this set cannot be studied directly, we can investigate it through its cohomological representation. The map $\theta: \mathcal{E}(X) \rightarrow \mathrm{O}(B)$, defined in Definition 6.15 (2), realizes this idea. Here $B$ is a lattice of rank three, which is essentially the same as the Mukai paring on the abelian surface of Picard number one, as defined in Definition 6.6. The group $\mathrm{O}(B)$ consists of the isometry of the lattice $B$.

The main result of this section, Theorem 6.16, claims the existence of bijection

$$
\operatorname{Im} \theta \cong G /\{ \pm 1\},
$$

where $G$ is a subgroup of $\operatorname{SL}(2, \mathbb{R})$ introduced in Definition 6.9, The element of $G$ encodes the data of Mukai vectors $\Phi((0,0,1))$ and $\Phi((1,0,0))$. The bijection (6.2) indicates that the cohomological action of $\Phi$ is uniquely determined by the image of the skyscraper sheaf and the structure sheaf. 
6.1. Cohomological correspondence. In this preliminary subsection we give a description of the Mukai lattice on the Fourier-Mukai partner $Y$ of $X$.

In the paper [Y3, $\S \S 1.3]$, one of the author deals with the ample divisor $\widehat{H}$. Let us recall the argument. For a while we do not assume $\operatorname{NS}(X)=\mathbb{Z} H$. Let $v_{0}=\left(r_{0}, \xi_{0}, a_{0}\right)$ be an isotropic Mukai vector and set $Y:=M_{X}^{H}\left(v_{0}\right)$. Assume that there exists a universal family $\mathbf{E}$ on $Y \times X$. Let $x \in X$ and $y \in Y$ be arbitrary closed points. Set $w_{0}:=v_{0}\left(\left.\mathbf{E}\right|_{\{x\} \times Y}\right)=r_{0}+\widetilde{\xi}_{0}+\widetilde{a}_{0} \rho_{Y}$, $\widetilde{\xi}_{0} \in \operatorname{NS}(Y)$. Then $\Phi\left(\left.\mathbf{E}^{\vee}\right|_{X \times\{y\}}\right)=\mathbb{C}_{y}$ and $\Phi\left(\mathbb{C}_{x}\right)=\left.\mathbf{E}\right|_{\{x\} \times Y}$, where $\Phi:=\Phi_{X \rightarrow Y}^{\mathbf{E}}$. Hence for the cohomological FMT, we get $\Phi^{H}\left(v_{0}^{\vee}\right)=\rho_{Y}$ and $\Phi^{H}\left(\rho_{X}\right)=w_{0}$.

Now we introduce a map $\nu: H^{2}(X, \mathbb{Q}) \rightarrow H^{2}(Y, \mathbb{Q})$ by

$$
-\nu(D):=-\left[\Phi^{H}\left(D+\frac{\left(D,-\xi_{0}\right)}{r_{0}} \rho_{X}\right)\right]_{1}=\left[p_{Y *}\left(\left(c_{2}(\mathbf{E})-\frac{r_{0}-1}{2 r_{0}} c_{1}(\mathbf{E})^{2}\right) \cup p_{X}^{*}(D)\right)\right]_{1},
$$

where [ ] $]_{1}$ means the natural projection from $H^{\mathrm{ev}}(Y, \mathbb{Q})$ to $H^{2}(Y, \mathbb{Q})$. Then by [Y3, Lemma 1.4], $-r_{0} \nu(H)$ is represented by an ample divisor on $Y$. Thus $\widehat{H}$ is proportional to $-r_{0} \nu(H)$.

Using this divisor $\widehat{H}$ we can describe the cohomological behaviour of FMT in the following manner.

Fact 6.1 ([Y3, Proposition 1.5]). (1) Every Mukai vector $v=(r, \xi, a) \in H^{\mathrm{ev}}(X, \mathbb{Z})_{\mathrm{alg}}$ is uniquely written as

with

$$
v=\ell v_{0}^{\vee}+\chi \rho_{X}+d H+D-\frac{1}{r_{0}}\left(d H+D, \xi_{0}\right) \rho_{X}
$$

$\ell:=\frac{r}{r_{0}} \in \frac{1}{r_{0}} \mathbb{Z}, \quad \chi:=\frac{r a_{0}+\left(\xi_{0}, \xi\right)+r_{0} a}{r_{0}} \in \frac{1}{r_{0}} \mathbb{Z}, \quad d:=\frac{\left(r_{0} \xi+r \xi_{0}, H\right)}{r_{0}\left(H^{2}\right)} \in \frac{1}{r_{0}\left(H^{2}\right)} \mathbb{Z}$

and $D \in \mathrm{NS}(X) \otimes_{\mathbb{Z}} \mathbb{Q} \cap H^{\perp}$.

$(2)$

$$
\begin{aligned}
& \left.\Phi^{H}\left(\ell v_{0}^{\vee}+\chi \rho_{X}+d H+D-\frac{1}{r_{0}}\left(d H+D, \xi_{0}\right) \rho_{X}\right)\right) \\
& =\ell \rho_{Y}+\chi w_{0}+d \nu(H)+\nu(D)+\frac{1}{r_{0}}\left(d \nu(H)+\nu(D), \widetilde{\xi}_{0}\right) \rho_{Y},
\end{aligned}
$$

where $D \in \mathrm{NS}(X) \otimes_{\mathbb{Z}} \mathbb{Q} \cap H^{\perp}$.

Now we return to the case $\operatorname{NS}(X)=\mathbb{Z} H$. Suppose that for an isotropic Mukai vector $v$, $Y:=M_{X}^{H}(v) \in \operatorname{FM}(X)$ and that there exists a universal family $\mathbf{E}$ on $Y \times X$. Set $\operatorname{NS}(Y)=\mathbb{Z} \widehat{H}$ and $\Phi:=\Phi_{X \rightarrow Y}^{\mathbf{E}}$.

Lemma 6.2. In this setting we have $-\nu(H)=\widehat{H}$.

Proof. We can write $\widehat{H}=-c \nu(H)$ with $c \in \mathbb{Q}_{>0}$ as above. Lemma 2.7 shows that $\left(\widehat{H}^{2}\right)=2 n$. Thus we have $c=1$. (See also [Y3, Lemma Appendix A.1] for a general treatment.)

Consider the action of the cohomological FMT $\Phi^{H}$ on the lattices $H^{\mathrm{ev}}(X, \mathbb{Z})_{\mathrm{alg}} \rightarrow H^{\mathrm{ev}}(Y, \mathbb{Z})_{\mathrm{alg}}$ of rank three. Take a basis $\left\langle 1, H, \rho_{X}\right\rangle$ of $H^{\mathrm{ev}}(X, \mathbb{Z})_{\text {alg }}$ and a basis $\left\langle 1, \widehat{H}, \rho_{Y}\right\rangle$ of $H^{\mathrm{ev}}(Y, \mathbb{Z})_{\text {alg }}$. Then $\Phi^{H}$ can be written by a $3 \times 3$ matrix.

Lemma 6.3. The determinant of $\Phi^{H}$ is 1 .

Proof. By Lemma 2.7, we may put $\left(H^{2}\right)=\left(\widehat{H}^{2}\right)=2 n$. Set $v_{0}=\left(r_{0}, d_{0} H, a_{0}\right)$ and $w_{0}=$ $\left(r_{0}, \widetilde{d}_{0} \widehat{H}, \widetilde{a}_{0}\right)$. From (6.3),$\Phi^{H}$ has an effect

$$
\ell v_{0}^{\vee}+d H+\left(\chi-\frac{2 n d_{0}}{r_{0}} d\right) \rho_{X} \mapsto \chi w_{0}-d \widehat{H}+\left(\ell-\frac{2 n \widetilde{d}_{0}}{r_{0}} d\right) \rho_{Y},
$$


so that if we present $\Phi^{H}$ by the basis $\left\langle v_{0}^{\vee}, H, \rho_{X}\right\rangle$ and $\left\langle w_{0}, \widehat{H}, \rho_{Y}\right\rangle$, then its matrix is

$$
M:=\left[\begin{array}{ccc}
0 & 2 n d_{0} / r_{0} & 1 \\
0 & -1 & 0 \\
1 & -2 n \widetilde{d}_{0} / r_{0} & 0
\end{array}\right] .
$$

On the other hand, the base change $\left\langle 1, H, \rho_{X}\right\rangle \mapsto\left\langle v_{0}^{\vee}, H, \rho_{X}\right\rangle$ can be written as

$$
P_{1}:=\left[\begin{array}{ccc}
1 / r_{0} & 0 & 0 \\
d_{0} / r_{0} & 1 & 0 \\
-a_{0} / r_{0} & 0 & 1
\end{array}\right]
$$

Similarly the base change $\left\langle w_{0}, \widehat{H}, \rho_{Y}\right\rangle \mapsto\left\langle 1, \widehat{H}, \rho_{Y}\right\rangle$ can be written as

$$
P_{2}:=\left[\begin{array}{ccc}
1 / r_{0} & 0 & 0 \\
-\widetilde{d}_{0} / r_{0} & 1 & 0 \\
-\widetilde{a}_{0} / r_{0} & 0 & 1
\end{array}\right]^{-1}
$$

The determinant of the multiplication of these three matrices is 1 . Thus the conclusion holds.

Remark 6.4. For later use, we display the resulting matrix $P_{2} M P_{1}$.

$$
P_{2} M P_{1}=\left[\begin{array}{ccc}
a_{0} & 2 n d_{0} & r_{0} \\
\left(a_{0} \widetilde{d}_{0}-d_{0}\right) / r_{0} & -1+2 n d_{0} \widetilde{d}_{0} / r_{0} & \widetilde{d}_{0} \\
\left(-2 n d_{0} \widetilde{d}_{0}+r_{0}+a_{0} \widetilde{a}_{0} r_{0}\right) / r_{0}^{2} & 2 n\left(b_{0} d_{0}-\widetilde{d}_{0}\right) / r_{0} & \widetilde{a}_{0}
\end{array}\right]
$$

6.2. Some calculations for matrix description. In this subsection we give elementary remarks on the groups appeared in the diagram (6.1). We begin with the description of the indefinite orthogonal group $\mathrm{O}(2,1)$. The following notation will be used.

$$
\begin{aligned}
& I_{2,1}:=\operatorname{diag}(1,1,-1) \in \mathrm{GL}(3, \mathbb{R}), \\
& \mathrm{O}(2,1):=\left\{g \in \mathrm{GL}(3, \mathbb{R}) \mid{ }^{t} g I_{2,1} g=I_{2,1}\right\}, \\
& \mathrm{SO}(2,1):=\mathrm{SL}(3, \mathbb{R}) \cap \mathrm{O}(2,1) .
\end{aligned}
$$

The connected components of $\mathrm{O}(2,1)$ are described as follows.

Lemma 6.5. There are four connected components of $\mathrm{O}(2,1)$, and these are given by

(0) $\left\{g=\left(g_{i j}\right) \in \mathrm{O}(2,1) \mid \operatorname{det} g=+1, g_{33} \geq 1\right\}$,

(1) $\left\{g=\left(g_{i j}\right) \in \mathrm{O}(2,1) \mid \operatorname{det} g=+1, g_{33} \leq-1\right\}$,

(2) $\left\{g=\left(g_{i j}\right) \in \mathrm{O}(2,1) \mid \operatorname{det} g=-1, g_{33} \geq 1\right\}$,

(3) $\left\{g=\left(g_{i j}\right) \in \mathrm{O}(2,1) \mid \operatorname{det} g=-1, g_{33} \leq-1\right\}$.

Proof. This is a well-known fact and we only show the sketch. First note that $g \in \mathrm{O}(2,1)$ has $\operatorname{det} g= \pm 1$. Introduce the bilinear form $(\cdot, \cdot)$ on $\mathbb{R}^{3}$ by

$$
(x, y):=x_{1} y_{1}+x_{2} y_{2}-x_{3} y_{3} .
$$

Then for $g \in \mathrm{GL}(3, \mathbb{R})$, the condition ${ }^{t} g I_{2,1} g=I_{2,1}$ is equivalent to $\left[\left(g_{i}, g_{j}\right)\right]=I_{2,1}$, where $g_{i}$ denotes the $i$-th column of the matrix $g$. Hence the condition is rewritten into
(i) $\left(g_{i}, g_{j}\right)=0(1 \leq i, j \leq 3, i \neq j)$,
(ii) $\left(g_{i}, g_{i}\right)=1(i=1,2)$,
(iii) $\left(g_{3}, g_{3}\right)=-1$.

Then (iii) implies $g_{13}^{2}+g_{23}^{2}-g_{33}^{2}=-1$, which yields $g_{33}^{2} \geq 1$.

Considering the continuous functions $\operatorname{det} g, g_{33}$ on the real Lie group $\mathrm{O}(2,1)$, we find that the subsets (0)-(3) belong to mutually different components. Define $I_{0}:=\operatorname{diag}(1,1,1), I_{1}:=$ $\operatorname{diag}(-1,1,-1), I_{2}:=\operatorname{diag}(1,-1,1), I_{3}:=\operatorname{diag}(1,1,-1)$. Then each $I_{j}$ belongs to the subset $(j)$. One can explicitly construct a path from any element of the subset $(j)$ to the element $I_{j}$. Thus the conclusion follows. 
The component (0) in the above classification will be denoted by $\mathrm{SO}_{0}(2,1)$, which contains the identity. As is well-known, the adjoint map

$$
\begin{array}{cl}
\overline{\mathrm{Ad}}: \quad \mathrm{SL}(2, \mathbb{R}) & \rightarrow \mathrm{SO}_{0}(2,1) \\
g & \mapsto\left(x \in \mathrm{sl}(2, \mathbb{R}) \mapsto g^{-1} x g\right)
\end{array}
$$

induces an anti-isomorphism $\operatorname{PSL}(2, \mathbb{R}):=\mathrm{SL}(2, \mathbb{R}) /\{ \pm 1\} \stackrel{\sim}{\rightarrow} \mathrm{SO}_{0}(2,1)$. Here the image $\overline{\mathrm{Ad}}(g)=$ $\left(x \mapsto g^{-1} x g\right)$ is regarded as the matrix presented in the basis $\left\langle\left[\begin{array}{cc}1 & 0 \\ 0 & -1\end{array}\right],\left[\begin{array}{ll}0 & 1 \\ 1 & 0\end{array}\right],\left[\begin{array}{cc}0 & 1 \\ -1 & 0\end{array}\right]\right\rangle$ of $\mathrm{sl}(2, \mathbb{R})$. A straightforward computation yields that for $g=\left[\begin{array}{ll}p & q \\ r & s\end{array}\right]$ the matrix becomes

$$
\overline{\operatorname{Ad}}(g)=\left[\begin{array}{ccc}
p s+q r & -p q+r s & p q+r s \\
q s-p r & \left(p^{2}-q^{2}-r^{2}+s^{2}\right) / 2 & \left(-p^{2}+q^{2}-r^{2}+s^{2}\right) / 2 \\
q s+p r & \left(-p^{2}-q^{2}+r^{2}+s^{2}\right) / 2 & \left(p^{2}+q^{2}+r^{2}+s^{2}\right) / 2
\end{array}\right]
$$

The overline in the notation indicates that we do not apply the usual definition $\operatorname{Ad}(g):=(x \mapsto$ $\left.g x g^{-1}\right)$, which gives an isomorphism $\operatorname{PSL}(2, \mathbb{R}) \stackrel{\sim}{\rightarrow} \mathrm{SO}_{0}(2,1)$. The quadratic form used in this correspondence is $x_{1}^{2}+x_{2}^{2}-x_{3}^{2}$, which is different from the Mukai paring. This discrepancy is solved as follows.

Definition 6.6. We define

$$
\operatorname{Sym}_{2}(\mathbb{R}):=\left\{\left[\begin{array}{ll}
x & y \\
y & z
\end{array}\right] \mid x, y, z \in \mathbb{R}\right\} .
$$

We also introduce a bilinear form $B$ on $\operatorname{Sym}_{2}(\mathbb{R})$ as

$$
B\left(X, X^{\prime}\right):=-\operatorname{tr}\left(\widetilde{X} X^{\prime}\right)=2 y y^{\prime}-\left(x z^{\prime}+z x^{\prime}\right), \quad X=\left[\begin{array}{ll}
x & y \\
y & z
\end{array}\right], X^{\prime}=\left[\begin{array}{ll}
x^{\prime} & y^{\prime} \\
y^{\prime} & z^{\prime}
\end{array}\right] \in \operatorname{Sym}_{2}(\mathbb{R}) .
$$

Here we denoted $\widetilde{X}:=\left[\begin{array}{cc}z & -y \\ -y & x\end{array}\right]$ for $X=\left[\begin{array}{ll}x & y \\ y & z\end{array}\right]$.

The signature of $(\operatorname{Sym}(2, \mathbb{R}), B)$ is $(2,1)$. We define an action of $\operatorname{GL}(2, \mathbb{R})$ on $\operatorname{Sym}_{2}(\mathbb{R})$ by

$$
X \mapsto X \cdot g:={ }^{t} g X g, \quad X \in \operatorname{Sym}_{2}(\mathbb{R}), g \in \mathrm{GL}(2, \mathbb{R}) .
$$

Since $\widetilde{X}=(\operatorname{det} X) X^{-1}$, we see that $\widetilde{{ }^{t} g X g}=(\operatorname{det} g)^{2} g^{-1} \cdot \widetilde{X} \cdot{ }^{t} g^{-1}$. Hence

$$
B\left({ }^{t} g X g,{ }^{t} g X^{\prime} g\right)=(\operatorname{det} g)^{2} B\left(X, X^{\prime}\right) .
$$

In particular, $\mathrm{SL}(2, \mathbb{R})$ preserves the bilinear form $B$. Thus we get a map $\alpha: \mathrm{SL}(2, \mathbb{R}) \rightarrow \mathrm{O}(B)$, where $\mathrm{O}(B)$ is the set of linear transformations preserving the paring $B$.

To present the homomorphism $\alpha$ explicitly, we choose a basis $\left\langle\left[\begin{array}{ll}1 & 0 \\ 0 & 0\end{array}\right],\left[\begin{array}{ll}0 & 1 \\ 1 & 0\end{array}\right],\left[\begin{array}{ll}0 & 0 \\ 0 & 1\end{array}\right]\right\rangle$ of $\operatorname{Sym}_{2}(\mathbb{R})$. Then the paring $B$ corresponds to the symmetric matrix

$$
J:=\left[\begin{array}{ccc}
0 & 0 & -1 \\
0 & 2 & 0 \\
-1 & 0 & 0
\end{array}\right]
$$

Using the same basis, we can regard the element $M \in \mathrm{O}(B)$ as a $3 \times 3$ matrix. There is an isomorphism $\beta: \mathrm{O}(B) \rightarrow \mathrm{O}(2,1)$ defined by

$$
\beta: \mathrm{O}(B) \ni M \mapsto P^{-1} M P \in \mathrm{O}(2,1),
$$


where we set

$$
P:=\frac{1}{\sqrt{2}}\left[\begin{array}{ccc}
-1 & 0 & 1 \\
0 & 1 & 0 \\
1 & 0 & 1
\end{array}\right]
$$

Note that the map $\beta$ is well-defined since ${ }^{t} P J P=I_{2,1}$ holds.

Lemma 6.7. The following diagram is commutative.

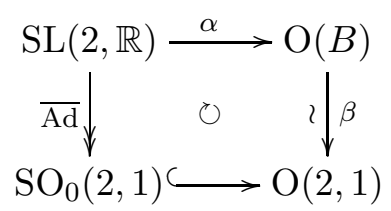

In particular the map $\beta \circ \alpha: \mathrm{SL}(2, \mathbb{Z}) \rightarrow \mathrm{O}(2,1)$ is a surjective anti-homomorphism $\mathrm{SL}(2, \mathbb{Z}) \rightarrow$ $\mathrm{SO}_{0}(2,1)$.

Proof. For $g=\left[\begin{array}{ll}p & q \\ r & s\end{array}\right] \in \mathrm{SL}(2, \mathbb{R})$, the image $\alpha(g)=\left(X \mapsto{ }^{t} g X g\right)$ in (6.7) is written in a matrix form via the basis $\left\langle\left[\begin{array}{ll}1 & 0 \\ 0 & 0\end{array}\right],\left[\begin{array}{ll}0 & 1 \\ 1 & 0\end{array}\right],\left[\begin{array}{ll}0 & 0 \\ 0 & 1\end{array}\right]\right\rangle$ of $\operatorname{Sym}_{2}(\mathbb{R})$ as

$$
\alpha(g):=\left[\begin{array}{ccc}
p^{2} & 2 p r & r^{2} \\
p q & p s+q r & r s \\
q^{2} & 2 q s & s^{2}
\end{array}\right] .
$$

It satisfies ${ }^{t} \alpha(g) J \alpha(g)=J$. Then a direct calculation using (6.6) and (6.10) yields the desired equality $\overline{\operatorname{Ad}}(g)=P^{-1} \alpha(g) P$.

We continue the study on $\operatorname{Sym}_{2}(\mathbb{R})$. Set

$$
\operatorname{Sym}_{2}(\mathbb{Z}, n):=\left\{\left[\begin{array}{cc}
x & y \sqrt{n} \\
y \sqrt{n} & z
\end{array}\right] \mid x, y, z \in \mathbb{Z}\right\} \subset \operatorname{Sym}_{2}(\mathbb{R}) .
$$

Then $B$ is an integral bilinear form on $\operatorname{Sym}_{2}(\mathbb{Z}, n)$. It is nothing but an analogue of the Mukai pairing for abelian surfaces of Picard number one.

Lemma 6.8. Let $x, y, z, w \in \mathbb{R}$ be algebraic numbers such that

(i) $x^{2}, x y / \sqrt{n}, y^{2} \in \mathbb{Z}$.

(ii) $z^{2}, z w / \sqrt{n}, w^{2} \in \mathbb{Z}$.

(iii) $x w-y z= \pm 1$.

Then there are unique integers $a, b, c, d$ and $r, s>0$ such that

$$
\left[\begin{array}{cc}
x & y \\
z & w
\end{array}\right]=\left[\begin{array}{ll}
a \sqrt{r} & b \sqrt{s} \\
c \sqrt{s} & d \sqrt{r}
\end{array}\right]
$$

with $r s=n$ and $a d r-b c s= \pm 1$.

Proof. The case $x y=0$ is trivial, since then we can write

$$
\left[\begin{array}{cc}
x & y \\
z & w
\end{array}\right]= \pm\left[\begin{array}{cc}
0 & 1 \\
-1 & d \sqrt{n}
\end{array}\right] \quad \text { or } \pm\left[\begin{array}{cc}
1 & 0 \\
c \sqrt{n} & 1
\end{array}\right], \quad c, d \in \mathbb{Z} .
$$

The case $z w=0$ can be treated similarly. Hence we may assume that $x y z w \neq 0$.

We set $a:=\operatorname{sgn}(x) \operatorname{gcd}\left(x^{2}, x y / \sqrt{n}\right)$ and $b:=\operatorname{sgn}(y) \operatorname{gcd}\left(y^{2}, x y / \sqrt{n}\right)$. The condition (iii) yields

$$
x^{2} w^{2}+y^{2} z^{2}-2 \frac{x y}{\sqrt{n}} \frac{z w}{\sqrt{n}} n=1 .
$$


Hence $\operatorname{gcd}\left(a, y^{2}\right)=1, \operatorname{gcd}\left(b, x^{2}\right)=1$, and $\operatorname{gcd}(a, b)=1$. We can write $x^{2}=a^{2} r, y^{2}=b^{2} s$ and $x y / \sqrt{n}=a b \lambda$, where $\lambda, r, s \in \mathbb{Z}$. Then $x^{2} y^{2}=(x y / \sqrt{n})^{2} n \Longleftrightarrow a^{2} r b^{2} s=a^{2} b^{2} \lambda^{2} n$ implies that $r s=\lambda^{2} n$. Since $|a|=\operatorname{gcd}\left(x^{2}, x y / \sqrt{n}\right)=\operatorname{gcd}\left(a^{2} r, a b \lambda\right)$, we have $\operatorname{gcd}(a r, b \lambda)=1$. In the same way, we get $\operatorname{gcd}(b s, a \lambda)=1$. Therefore we should have $\lambda= \pm 1$ and $r s=n$. A similar argument using (ii) and (iii) works on $z, w$.

In the consequence we have

$$
\left[\begin{array}{cc}
x & y \\
z & w
\end{array}\right]=\left[\begin{array}{cc}
a \sqrt{r} & b \sqrt{s} \\
c \sqrt{t} & d \sqrt{u}
\end{array}\right], \quad a, b, c, d \in \mathbb{Z}, r, s, t, u \in \mathbb{Z}_{>0}, r s=t u=n .
$$

Then (6.12) becomes $a^{2} d^{2} r u+b^{2} c^{2} s t-2 a b c d n=1$. By $r s=t u=n$, we have $\operatorname{gcd}(s, u)=$ $\operatorname{gcd}(t, r)=1$. Hence we have $s=t$ and $r=u$. Then (iii) is equivalent to the requirement $a d r-b c s= \pm 1$.

Finally we prove the uniqueness. By $a d r-b c s= \pm 1$ we have $\operatorname{gcd}\left(x^{2}, x y / \sqrt{n}\right)=|a| \operatorname{gcd}(a r, b)=$ $|a|$. Since the sign of $a$ should equal to that of $x, a$ is uniquely determined. In the same way, $b, c, d$ are uniquely determined. Then $r$ and $s$ are characterized as $s=x^{2} / a^{2}$ and $r=y^{2} / b^{2}$. Therefore the uniqueness is shown.

The condition appeared in Lemma 6.8 defines a subgroup of $\mathrm{SL}(2, \mathbb{R})$.

Definition 6.9. We set

$$
\begin{aligned}
& \widehat{G}:=\left\{\left[\begin{array}{cc}
x & y \\
z & w
\end{array}\right] \in \operatorname{GL}(2, \mathbb{R}) \mid x^{2}, y^{2}, z^{2}, w^{2}, \frac{x y}{\sqrt{n}}, \frac{z w}{\sqrt{n}} \in \mathbb{Z}\right\}, \\
& G:=\widehat{G} \cap \operatorname{SL}(2, \mathbb{R}) .
\end{aligned}
$$

Lemma 6.10. (1) $G$ (resp. $\widehat{G})$ is a subgroup of $\operatorname{SL}(2, \mathbb{R})($ resp. $\operatorname{GL}(2, \mathbb{R}))$.

(2)

$$
\begin{aligned}
& \widehat{G}=G \rtimes\left\langle\left[\begin{array}{cc}
1 & 0 \\
0 & -1
\end{array}\right]\right\rangle \\
& {\left[\begin{array}{cc}
1 & 0 \\
0 & -1
\end{array}\right]\left[\begin{array}{cc}
x & y \\
z & w
\end{array}\right]\left[\begin{array}{cc}
1 & 0 \\
0 & -1
\end{array}\right]=\left[\begin{array}{cc}
x & -y \\
-z & w
\end{array}\right], \quad\left[\begin{array}{cc}
x & y \\
z & w
\end{array}\right] \in \mathrm{GL}(2, \mathbb{R}) .}
\end{aligned}
$$

(3) $\widehat{G}$ preserves $\operatorname{Sym}_{2}(\mathbb{Z}, n)$ under the action (6.7) of $\operatorname{GL}(2, \mathbb{R})$ on $\operatorname{Sym}_{2}(\mathbb{R})$.

Proof. We note that

$$
\left[\begin{array}{ll}
a \sqrt{r} & b \sqrt{s} \\
c \sqrt{s} & d \sqrt{r}
\end{array}\right]\left[\begin{array}{ll}
a^{\prime} \sqrt{r^{\prime}} & b^{\prime} \sqrt{s^{\prime}} \\
c^{\prime} \sqrt{s^{\prime}} & d^{\prime} \sqrt{r^{\prime}}
\end{array}\right]=\left[\begin{array}{ll}
a a^{\prime} \sqrt{r r^{\prime}}+b c^{\prime} \sqrt{s s^{\prime}} & a b^{\prime} \sqrt{r s^{\prime}}+b d^{\prime} \sqrt{s r^{\prime}} \\
c a^{\prime} \sqrt{s r^{\prime}}+d c^{\prime} \sqrt{r s^{\prime}} & c b^{\prime} \sqrt{s s^{\prime}}+d d^{\prime} \sqrt{r r^{\prime}}
\end{array}\right]
$$

for two elements of $\widehat{G}$. Since $\sqrt{s r}=\sqrt{s^{\prime} r^{\prime}}=\sqrt{n}, \widehat{G}$ is closed under the multiplication. Obviously the inverse is also well-defined on $\widehat{G}$. Hence $\widehat{G}$ is a subgroup of $\operatorname{SL}(2, \mathbb{R})$.

(2) is obvious and (3) is a consequence of the following calculation.

$$
\begin{aligned}
& {\left[\begin{array}{ll}
a \sqrt{r} & c \sqrt{s} \\
b \sqrt{s} & d \sqrt{r}
\end{array}\right]\left[\begin{array}{cc}
x & y \sqrt{n} \\
y \sqrt{n} & z
\end{array}\right]\left[\begin{array}{cc}
a \sqrt{r} & b \sqrt{s} \\
c \sqrt{s} & d \sqrt{r}
\end{array}\right] } \\
= & {\left[\begin{array}{cc}
a^{2} r x+2 a c n y+c^{2} s z & (a b x+(a d r+b c s) y+c d z) \sqrt{n} \\
(a b x+(a d r+b c s) y+c d z) \sqrt{n} & b^{2} s x+2 b d n y+d^{2} r z
\end{array}\right] . }
\end{aligned}
$$

Remark 6.11. If $\left(H^{2}\right)=2$, that is, if the surface is principally polarized, then $G=\mathrm{SL}(2, \mathbb{Z})$. This group is mentioned in [M8, Example 1.15 (1)].

For stating Lemma 6.13, we introduce a terminology in $\operatorname{Sym}_{2}(\mathbb{R})$, corresponding to the notion of the positive Mukai vector. 
Definition 6.12. An element $\left[\begin{array}{ll}x & y \\ y & z\end{array}\right] \in \operatorname{Sym}_{2}(\mathbb{R})$ is called positive if $x>0$, or if $x=0$ and $y>0$, or if $x=y=0$ and $z>0$.

Lemma 6.13. Consider the action (6.7) of $\operatorname{PSL}(2, \mathbb{R})$ on $\operatorname{Sym}_{2}(\mathbb{Z}, n)$.

(1) If $\gamma_{1}, \gamma_{2} \in \operatorname{PSL}(2, \mathbb{R})$ have the same action on $\left[\begin{array}{ll}1 & 0 \\ 0 & 0\end{array}\right]$ and $\left[\begin{array}{ll}0 & 0 \\ 0 & 1\end{array}\right]$, then $\gamma_{1}=\gamma_{2}$.

(2) Assume that we are given two positive elements $X_{1}, X_{2} \in \operatorname{Sym}_{2}(\mathbb{Z}, n)$ with $B\left(X_{1}, X_{1}\right)=$ $B\left(X_{2}, X_{2}\right)=0$ and $B\left(X_{1}, X_{2}\right)=-1$. Then there exists a unique element $\gamma \in G /\{ \pm 1\} \subset$ $\operatorname{PSL}(2, \mathbb{R})$ such that $\left[\begin{array}{ll}1 & 0 \\ 0 & 0\end{array}\right] \cdot \gamma=X_{1}$ and $\left[\begin{array}{ll}0 & 0 \\ 0 & 1\end{array}\right] \cdot \gamma=X_{2}$.

Proof. (1) Take elements $g_{1}:=\left[\begin{array}{ll}x & y \\ z & w\end{array}\right], g_{2}:=\left[\begin{array}{ll}p & q \\ r & s\end{array}\right] \in \mathrm{SL}(2, \mathbb{R})$ such that their equivalent classes in $\operatorname{PSL}(2, \mathbb{R})$ equal to $\gamma_{1}, \gamma_{2}$. Then the condition is rewritten into

$$
{ }^{t} g_{1}\left[\begin{array}{ll}
1 & 0 \\
0 & 0
\end{array}\right] g_{1}={ }^{t} g_{2}\left[\begin{array}{ll}
1 & 0 \\
0 & 0
\end{array}\right] g_{2}, \quad{ }^{t} g_{1}\left[\begin{array}{ll}
0 & 0 \\
0 & 1
\end{array}\right] g_{1}={ }^{t} g_{2}\left[\begin{array}{ll}
0 & 0 \\
0 & 1
\end{array}\right] g_{2} .
$$

These equations yield $x^{2}=p^{2}, x y=p q, y^{2}=q^{2}, z^{2}=r^{2}, z w=r s, w^{2}=s^{2}$. Hence $(x, y)=$ $\epsilon_{1}(p, q)$ and $(z, w)=\epsilon_{2}(r, s)$ where $\epsilon_{1}=1$ or -1 and $\epsilon_{2}=1$ or -1 . Since $g_{1}, g_{2} \in \operatorname{SL}(2, \mathbb{R})$, we should have $\epsilon_{1}=\epsilon_{2}$. Therefore $g_{1}= \pm g_{2}$, which means $\gamma_{1}=\gamma_{2}$.

(2) The uniqueness follows from (1). We only have to show the existence.

Set $X_{i}=\left[\begin{array}{cc}r_{i} & d_{i} \sqrt{n} \\ d_{i} \sqrt{n} & a_{i}\end{array}\right](i=1,2)$. Then the assumption is equivalent to

$$
d_{1}^{2} n-r_{1} a_{1}=0, \quad d_{2}^{2} n-r_{2} a_{2}=0, \quad 2 d_{1} d_{2} n-r_{1} a_{2}-r_{2} a_{1}=-1 .
$$

By the first two equations we can take $x, y, z, w \in \mathbb{R}$ such that $x^{2}=r_{1}, x y / \sqrt{n}=d_{1}, y^{2}=a_{1}$, $z^{2}=r_{2}, z w / \sqrt{n}=d_{2}, w^{2}=a_{2}$. By the third equation we also have $x w-y z= \pm 1$. Hence we may assume $x w-y z=+1$ by changing the signs of $z$ and $w$. Now set $g:=\left[\begin{array}{ll}x & y \\ z & w\end{array}\right]$. Then $g \in G \subset \operatorname{SL}(2, \mathbb{R})$. Then the equivalent class $\gamma:= \pm g \in \operatorname{PSL}(2, \mathbb{Z})$ can be defined. This $\gamma$ satisfies the requirement by construction.

6.3. Matrix description. We introduce a description of Mukai vectors via $2 \times 2$ matrix form, which is a generalization of the one defined in [M3]. We suppose $\operatorname{NS}(X)=\mathbb{Z} H$ and set $n:=$ $\left(H^{2}\right) / 2 \in \mathbb{Z}_{>0}$.

First we define an isomorphism of modules

$$
\begin{aligned}
& \iota_{X}: \quad H^{\mathrm{ev}}(X, \mathbb{Z})_{\text {alg }} \stackrel{\sim}{\rightarrow} \operatorname{Sym}_{2}(\mathbb{Z}, n) \\
& (r, d H, a) \quad \mapsto\left[\begin{array}{cc}
r & d \sqrt{n} \\
d \sqrt{n} & a
\end{array}\right] .
\end{aligned}
$$

Then it is clear that

$$
\left\langle v, v^{\prime}\right\rangle=B\left(\iota_{X}(v), \iota_{X}\left(v^{\prime}\right)\right) .
$$

Thus we have an isometry of lattices:

$$
\iota_{X}:\left(H^{\mathrm{ev}}(X, \mathbb{Z})_{\mathrm{alg}},\langle\cdot, \cdot\rangle\right) \stackrel{\sim}{\rightarrow}\left(\operatorname{Sym}_{2}(\mathbb{Z}, n), B\right) .
$$

We also have the next restatement of Lemma 6.8 and Lemma 6.13 ,

Lemma 6.14. If $X$ is an abelian surface with $\mathrm{NS}(X)=\mathbb{Z} H$ and $\left(H^{2}\right)=2 n$, then any pair of positive primitive isotropic Mukai vectors $v_{1}, v_{2} \in H^{\mathrm{ev}}(X, \mathbb{Z})_{\text {alg }}$ satisfying $\left\langle v_{1}, v_{2}\right\rangle=-1$ can be uniquely written as

$$
v_{1}=\left(a^{2} r, a b H, b^{2} s\right), \quad v_{2}=\left(c^{2} s, c d H, d^{2} r\right),
$$




$$
r s=n, \quad a d r-b c s=1, \quad a>0 .
$$

Proof. This can be shown in the same manner as Lemma 6.13(2), so we omit the detail.

Definition 6.15. (1) For $Y, Z \in \operatorname{FM}(X)$, we set

$$
\begin{aligned}
\mathrm{Eq}_{0}(\mathbf{D}(Y), \mathbf{D}(Z)) & :=\left\{\Phi_{Y \rightarrow Z}^{\mathbf{E}[2 k]} \in \operatorname{Eq}(\mathbf{D}(Y), \mathbf{D}(Z)) \mid \mathbf{E} \in \operatorname{Coh}(Y \times Z), k \in \mathbb{Z}\right\}, \\
\mathcal{E}(Z) & :=\bigcup_{Y \in \operatorname{FM}(X)} \operatorname{Eq}_{0}(\mathbf{D}(Y), \mathbf{D}(Z)), \\
\mathcal{E} & :=\bigcup_{Y, Z \in \mathrm{FM}(X)} \operatorname{Eq}_{0}(\mathbf{D}(Y), \mathbf{D}(Z)) .
\end{aligned}
$$

(2) Let $Y, Z \in \operatorname{FM}(X)$. For a $\operatorname{FMT} \Phi:=\Phi_{Y \rightarrow Z}^{\mathbf{E}^{\bullet}}: \mathbf{D}(Y) \rightarrow \mathbf{D}(Z)$ with $\mathbf{E}^{\bullet} \in \mathbf{D}(Y \times Z)$, set

$$
\theta(\Phi):=\iota_{Z} \circ \Phi^{H} \circ \iota_{Y}^{-1},
$$

where $\Phi^{H}: H^{\mathrm{ev}}(Y, \mathbb{Z}) \rightarrow H^{\mathrm{ev}}(Z, \mathbb{Z})$ is the cohomological FMT induced by $\Phi$ (see $\S \S 2.2$ ).

Note that $\mathcal{E}=\bigcup_{Z \in \mathrm{FM}(X)} \mathcal{E}(Z)$ and that $\mathcal{E}$ is a groupoid with respect to the composition of the equivalences. Since $\iota_{Y}, \iota_{Z}$ and $\Phi^{H}$ are isometries, their composition $\theta(\Phi)$ is again an isometry on the lattice $\left.\operatorname{SSym}_{2}(\mathbb{Z}, n), B\right)$. Thus we have a map $\theta: \mathcal{E} \rightarrow \mathrm{O}(B)$.

Theorem 6.16. For each $Z \in \operatorname{FM}(X)$, we have a bijection

$$
\theta(\mathcal{E}(Z)) \cong G /\{ \pm 1\}
$$

Proof. It is enough to show for the case $Z=X$. First we use Lemma 6.13 to make a map

$$
\kappa: \theta(\mathcal{E}(X)) \longrightarrow G /\{ \pm 1\}
$$

Take an element $\Phi=\Phi_{Y \rightarrow X}^{\mathbf{E}[2 k]} \in \operatorname{Eq}(\mathbf{D}(Y), \mathbf{D}(X))$. By Fact 2.6 (3), there are primitive isotropic Mukai vectors $v_{i}=\left(r_{i}, d_{i} H, a_{i}\right) \in H^{\mathrm{ev}}(X, \mathbb{Z})_{\text {alg }}$ for $i=1,2$ such that $v\left(\mathbf{E}_{y}\right)=v_{2}, Y \cong M_{X}^{H}\left(v_{2}\right)$, $\left\langle v_{1}, v_{2}\right\rangle=-1$ and $\mathbf{E}$ is a universal family on $Y \times X$. Set $X_{i}:=\iota_{X}\left(v_{i}\right) \in \operatorname{Sym}_{2}(\mathbb{Z}, n)$. Then the assumption of Lemma 6.13 (2) holds. Thus a map $\kappa$ is defined. The same Lemma 6.13 implies that $\kappa$ is injective.

For the converse relation, let us take $\gamma \in G /\{ \pm 1\}$ and choose a representative $g:=\left[\begin{array}{cc}x & y \\ z & w\end{array}\right] \in G$ of $\gamma$. Then by Lemma 6.8 there exists integers $a, b, c, d, r, s$ uniquely determined by $g$. If we set $v_{1}:=\left(a^{2} r, a b H, b^{2} s\right)$ and $v_{2}:=\left(c^{2} s, c d H, d^{2} r\right)$, then $\left\langle v_{1}^{2}\right\rangle=\left\langle v_{2}^{2}\right\rangle=0$ and $\left\langle v_{1}, v_{2}\right\rangle=$ $-(a d r-b c s)^{2}=-1$. Thus we can apply Proposition 2.14 (4) to the pair $v_{1}, v_{2}$. Hence there exists a FMT $\Psi:=\Phi_{X \rightarrow Y}^{\mathbf{E}^{\vee}[2]}$ such that $\Psi(E)=\mathbb{C}_{y}$ and $\Psi(F)=\mathcal{O}_{Y}[-i](i=0$ or 2$)$ for $E \in M_{X}^{H}\left(v_{2}\right)$ and $F \in M_{X}^{H}\left(v_{1}\right)$. Then the inverse transform $\Phi:=\Phi_{Y \rightarrow X}^{\mathbf{E}}$ satisfies $\Phi^{H}((1,0,0))=v_{1}$ and $\Phi^{H}((0,0,1))=v_{2}$. Set $X_{i}:=\iota_{X}\left(v_{i}\right)$. Now using 6.11, we find that $\theta(\Phi)$ and $\gamma$ have the same action on $X_{1}$ and $X_{2}$. Since $X_{1}$ and $X_{2}$ satisfy the assumption on Lemma 6.13 (1), we find that $\kappa \circ \theta\left(\Phi_{Y \rightarrow X}^{\mathbf{E}}\right)$ coincides with $\gamma$.

Remark 6.17. Lemma 6.3 means that the image $\beta \circ \theta(\Phi) \in \mathrm{O}(2,1)$ is actually in $\mathrm{SO}(2,1)$. Theorem 6.16 further claims that $\beta \circ \theta(\Phi) \in \mathrm{SO}_{0}(2,1)$. We can prove it by using Lemma 6.5 as follows. The matrix form of $\theta(\Phi)$ in the basis $\left\langle\left[\begin{array}{ll}1 & 0 \\ 0 & 0\end{array}\right],\left[\begin{array}{ll}0 & 1 \\ 1 & 0\end{array}\right],\left[\begin{array}{ll}0 & 0 \\ 0 & 1\end{array}\right]\right\rangle$ of $\operatorname{Sym}_{2}(\mathbb{R})$ is the $P_{2} M P_{1}$ in (6.4) (see Remark 6.4). The element $\beta \circ \theta(\Phi)$ is a matrix presented in the basis $\left\langle\left[\begin{array}{cc}1 & 0 \\ 0 & -1\end{array}\right],\left[\begin{array}{ll}0 & 1 \\ 1 & 0\end{array}\right],\left[\begin{array}{cc}0 & 1 \\ -1 & 0\end{array}\right]\right\rangle$ of $\operatorname{sl}(2, \mathbb{R})$. The transition matrix between these two bases is the $P$ 
in (6.9). Thus we should calculate $\beta \circ \theta(\Phi)=Q:=P^{-1} P_{2} M P_{1} P$. A straightforward computation yields

$$
\begin{aligned}
Q_{33} & =\frac{1}{2 r_{0}^{2}}\left(r_{0}+r_{0}^{3}+a_{0} r_{0}^{2}+\widetilde{a}_{0} r_{0}^{2}+a_{0} \widetilde{a}_{0} r_{0}-2 n d_{0} \widetilde{d}_{0}\right) \\
& =\frac{1}{2 r_{0}^{2}}\left(n\left(d_{0}-\widetilde{d}_{0}\right)^{2}+n\left(r_{0}-1\right) d_{0}^{2}+n\left(r_{0}-1\right) \widetilde{d}_{0}^{2}+\left(a_{0} \widetilde{a}_{0}+1\right) r_{0}\right)
\end{aligned}
$$

Since all the $a_{0}, \widetilde{a}_{0}, r_{0}$ are positive, we find that $Q_{33}>0$. Since we have known $\beta \circ \theta(\Phi) \in \operatorname{SO}(2,1)$, Lemma 6.5 shows that $\beta \circ \theta(\Phi) \in \mathrm{SO}_{0}(2,1)$

Next we treat the composition of FMT and the dualizing functor. For a FMT $\Phi_{X \rightarrow Y}^{\mathbf{E}^{\bullet}} \in$ $\operatorname{Eq}(\mathbf{D}(X), \mathbf{D}(Y))$, we set

$$
\theta\left(\Phi_{X \rightarrow Y}^{\mathbf{E}} \mathcal{D}_{X}\right):=\iota_{Y} \circ\left(\Phi_{X \rightarrow Y}^{\mathbf{E}}\right)^{H} \mathcal{D}_{X} \circ \iota_{X}^{-1}: \operatorname{Sym}_{2}(\mathbb{R}) \rightarrow \operatorname{Sym}_{2}(\mathbb{R}) .
$$

Here the $\mathcal{D}_{X}$ in the last line means the cohomological action $H^{\mathrm{ev}}(X, \mathbb{Z})_{\mathrm{alg}} \rightarrow H^{\mathrm{ev}}(X, \mathbb{Z})_{\mathrm{alg}}$ of the dualizing functor.

Lemma 6.18.

$$
\theta\left(\mathcal{D}_{X}\right)= \pm\left[\begin{array}{cc}
1 & 0 \\
0 & -1
\end{array}\right] \in \operatorname{PSL}(2, \mathbb{R})
$$

We also have $\theta\left(\Phi_{X \rightarrow Y}^{\mathbf{E}} \mathcal{D}_{X}\right) \in \operatorname{PSL}(2, \mathbb{R})$ for a $\operatorname{FMT} \Phi_{X \rightarrow Y}^{\mathbf{E}^{\bullet}} \in \operatorname{Eq}(\mathbf{D}(X), \mathbf{D}(Y))$.

(2) If $\theta\left(\Phi_{X \rightarrow Y}^{\mathbf{E}}\right)=\left[\begin{array}{ll}a & b \\ c & d\end{array}\right]$, then

$$
\theta\left(\Phi_{Y \rightarrow X}^{\mathbf{E}}\right)= \pm\left[\begin{array}{cc}
d & b \\
c & a
\end{array}\right], \quad \theta\left(\Phi_{Y \rightarrow X}^{\mathbf{E}^{\vee}[2]}\right)= \pm\left[\begin{array}{cc}
d & -b \\
-c & a
\end{array}\right], \quad \theta\left(\Phi_{X \rightarrow Y}^{\mathbf{E}^{\vee}[2]}\right)= \pm\left[\begin{array}{cc}
a & -b \\
-c & d
\end{array}\right] .
$$

Proof. (1) This is trivial.

(2) The claims follows from the following relations.

$$
\begin{aligned}
& \Phi_{Y \rightarrow X}^{\mathbf{E}^{\vee}[2]}=\left(\Phi_{X \rightarrow Y}^{\mathbf{E}}\right)^{-1}, \\
& \Phi_{Y \rightarrow X}^{\mathbf{E}}=\mathcal{D}_{X} \Phi_{Y \rightarrow X}^{\mathbf{E}^{\vee}[2]} \mathcal{D}_{Y}=\mathcal{D}_{X}\left(\Phi_{X \rightarrow Y}^{\mathbf{E}}\right)^{-1} \mathcal{D}_{Y}, \\
& \Phi_{X \rightarrow Y}^{\mathbf{E}^{\vee}[2]}=\left(\Phi_{Y \rightarrow X}^{\mathbf{E}}\right)^{-1}=\mathcal{D}_{Y} \Phi_{X \rightarrow Y}^{\mathbf{E}} \mathcal{D}_{X} .
\end{aligned}
$$

The first equality is obvious and the second one is a consequence of the Serre duality (Lemma2.8). For the third equality, we take the inverse of the second equality.

Now we summarize the argument of this section in the following manner.

Proposition 6.19. Suppose $\mathrm{NS}(X)=\mathbb{Z} H$ and set $n:=\left(H^{2}\right) / 2 \in \mathbb{Z}_{>0}$. Consider a pair of primitive isotropic Mukai vectors $v_{1}$ and $v_{2}$ on $X$ such that $\left\langle v_{1}, v_{2}\right\rangle=-1$. By Lemma 6.14, we may put

$$
\begin{aligned}
& v_{1}=\left(p_{1}^{2} r_{1}, p_{1} q_{1} H, q_{1}^{2} r_{2}\right), \quad v_{2}=\left(p_{2}^{2} r_{2}, p_{2} q_{2} H, q_{2}^{2} r_{1}\right), \\
& r_{1} r_{2}=n, \quad p_{1} q_{2} r_{1}-p_{2} q_{1} r_{2}=1, \quad p_{1}>0 .
\end{aligned}
$$

Let us denote by $E_{i}$ a simple semi-homogeneous sheaf with Mukai vector $v_{i}$, where $i=1,2$. Proposition 2.14 shows that there exists a FMT $\Phi:=\Phi_{Y \rightarrow X}^{\mathbf{E}}$ which converts the structure sheaf $\mathcal{O}_{Y}$ into $E_{1}$ and a skyscraper sheaf into $E_{2}$ up to even shift.

Then the action of the cohomological FMT $\Phi^{H}: H^{\mathrm{ev}}(Y, \mathbb{Z})_{\text {alg }} \rightarrow H^{\mathrm{ev}}(X, \mathbb{Z})_{\text {alg }}$ can be written as follows. Let $\widehat{H}$ be the ample generator of $\mathrm{NS}(Y)$. For a Mukai vector $v=(r, d \widehat{H}, a) \in$ $H^{\mathrm{ev}}(Y, \mathbb{Z})_{\mathrm{alg}}$, the image $\Phi^{H}(v)$ is given by

$$
\Phi^{H}(v)=v \cdot g, \quad g:=\left[\begin{array}{ll}
p_{1} \sqrt{r_{1}} & q_{1} \sqrt{r_{2}} \\
p_{2} \sqrt{r_{2}} & q_{2} \sqrt{r_{1}}
\end{array}\right] \in G .
$$


Here the action $\cdot$ is given by

$$
(r, d H, a) \cdot g:=\left(r^{\prime}, d^{\prime} H, a^{\prime}\right), \quad{ }^{t} g\left[\begin{array}{cc}
r & d \sqrt{n} \\
d \sqrt{n} & a
\end{array}\right] g=\left[\begin{array}{cc}
r^{\prime} & d^{\prime} \sqrt{n} \\
d^{\prime} \sqrt{n} & a^{\prime}
\end{array}\right] .
$$

Conversely, given $\gamma \in G /\{ \pm 1\}$, there exists a FMT $\Phi=\Phi_{Y \rightarrow X}^{\mathbf{E}}$ such that $\theta(\Phi)=\gamma$, and this FMT is unique up to even shifts, automorphism of $X$ and tensor with a degree zero line bundle.

\section{TAME COMPleX}

This section gives some examples of calculations involving the semi-homogeneous presentation and the matrix description. We consider a certain sequence of FMTs associated to the power of a quadratic matrix. The resulting images of the original sheaf defines a sequence of complexes, which we call tame complexes. These complexes are easy to handle in the sense that we can state explicit numerical criteria for a FMT to transform the complex into a stable sheaf. The criteria, which are the main results of this section, are given in Theorems 7.21 and 7.31 ,

We fix an abelian surface $X$ with $\operatorname{NS}(X)=\mathbb{Z} H$. We define $n:=\left(H^{2}\right) / 2 \in \mathbb{Z}_{>0}$.

7.1. The arithmetic group $G$ and numerical solutions for the ideal sheaf. Before starting the calculation, we notice the next result on the number of numerical solutions for (3.2).

Proposition 7.1. Let $E$ be a stable sheaf on $X$ with $\ell:=\left\langle v(E)^{2}\right\rangle / 2>0$. Then the numerical equation (3.2) for the Mukai vector $v:=v(E)$ gives the next criterion.

If $n \ell$ is a square number, then the number of numerical solution is 0 or 1 .

Otherwise, the number of numerical solutions is 0 or infinity.

Proof. We may assume that there is at least one solution for $v$. Then there exists a FMT $\Phi$ whose cohomological counterpart $\Phi^{H}$ maps $v$ to $(1,0,-\ell)$, and it is sufficient to prove the claim for $v=(1,0,-\ell)$.

We rewrite the numerical equation as $v= \pm\left(\ell u_{1}-u_{2}\right)$, where $u_{1}, u_{2}$ are positive isotropic Mukai vectors with $\left\langle u_{1}, u_{2}\right\rangle=-1$. Using Lemma 6.14, we can further rewrite the numerical equation as

$$
(1,0,-\ell)= \pm\left(\ell u_{1}-u_{2}\right), \quad u_{1}=\left(p_{1}^{2} r_{1}, p_{1} q_{1} H, q_{1}^{2} a_{1}\right), \quad\left\langle u_{2}^{2}\right\rangle=0,
$$

where $p_{1}, q_{1} \in \mathbb{Z}, r_{1}, a_{1} \in \mathbb{Z}_{>0}$ and $r_{1} a_{1}=n$. Then we have

$$
\begin{aligned}
0 & =\left\langle u_{2}^{2}\right\rangle=\left\langle\left(\ell p_{1}^{2} r_{1} \mp 1, \ell p_{1} q_{1} H, \ell\left(q_{1}^{2} a_{1} \pm 1\right)\right\rangle^{2}\right. \\
& =2 n\left(\ell p_{1} q_{1}\right)^{2}-2\left(\ell p_{1}^{2} r_{1} \mp 1\right) \ell\left(q_{1}^{2} a_{1} \pm 1\right) \\
& =2 n \ell^{2} p_{1}^{2} q_{1}^{2}-2 \ell\left(n \ell p_{1}^{2} q_{1}^{2} \mp q_{1}^{2} a_{1} \pm \ell p_{1}^{2} r_{1}-1\right) \\
& =2 \ell\left( \pm q_{1}^{2} a_{1} \mp \ell p_{1}^{2} r_{1}+1\right) .
\end{aligned}
$$

Therefore the above equation becomes

$$
\ell p_{1}^{2} r_{1}-q_{1}^{2} a_{1}= \pm 1 .
$$

Let us consider the solution of this equation. Suppose that $n \ell$ is a square number. We may suppose $\operatorname{gcd}\left(\ell r_{1}, a_{1}\right)=1$. Then since $\ell r_{1} a_{1}=\ell n$ is a square number, both $\ell r_{1}$ and $a_{1}$ are square numbers. Hence the equation above becomes $\left(\sqrt{\ell r_{1}} p_{1}-\sqrt{a_{1}} q_{1}\right)\left(\sqrt{\ell r_{1}} p_{1}+\sqrt{a_{1}} q_{1}\right)= \pm 1$. Then the solution is as follows.

$$
\begin{array}{ll}
\ell u_{1}-u_{2}=(-1,0, \ell): & u_{1}=(0,0,1), u_{2}=(1,0,0), \\
\ell u_{1}-u_{2}=(1,0,-\ell): & \text { a solution exists only when } \ell=1 \text { and } \\
& u_{1}=(1,0,0), u_{2}=(0,0,1) .
\end{array}
$$

Next suppose that $n \ell$ is not a square number. Put $r_{1}=n$ and $a_{1}=1$. Then by the above calculation, the equation $\ell u_{1}-u_{2}=(-1,0, \ell)$ leads to $q_{1}^{2}-\ell n p_{1}^{2}=1$, which has infinite number of solutions. 
Example 7.2. If $\operatorname{rk}(v)=0$, then we can write $v=(0, d H, a)$ with $\ell n=(n d)^{2}$. In this case, there is no kernel presentation.

Hereafter we assume that $\sqrt{\ell n} \notin \mathbb{Z}$. By Theorem [3.6, a general member of $M_{X}^{H}(v)$ has both kernel and cokernel presentations if a numerical solution exists.

Our next task is to describe the numerical solution of the ideal sheaf of 0-dimensional subscheme. First we introduce an arithmetic group $S_{n, \ell}$.

Definition 7.3. For $(x, y) \in \mathbb{R}^{2}$, set

We also set

$$
P(x, y):=\left[\begin{array}{cc}
y & \ell x \\
x & y
\end{array}\right]
$$

$$
S_{n, \ell}:=\left\{\left[\begin{array}{cc}
y & \ell x \\
x & y
\end{array}\right] \mid x^{2}, y^{2}, \frac{x y}{\sqrt{n}} \in \mathbb{Z}, y^{2}-\ell x^{2}= \pm 1\right\} .
$$

Lemma 7.4. (1) $S_{n, \ell}$ is a commutative subgroup of $\mathrm{GL}(2, \mathbb{R})$.

(2) We have a homomorphism

$$
\phi: \begin{array}{ccc}
S_{n, \ell} & \longrightarrow & \mathbb{R}^{\times} \\
P(x, y) & \mapsto y+x \sqrt{\ell} .
\end{array}
$$

(3) For $\ell>1 \phi$ is injective, and for $\ell=1$ we have

$$
\operatorname{Ker} \phi=\left\langle\left[\begin{array}{ll}
0 & 1 \\
1 & 0
\end{array}\right]\right\rangle
$$

(4) We set a subgroup $G_{n, \ell}$ of $\widehat{G}$ (Definition 6.9) to be

$$
G_{n, \ell}:=\left\{g \in \widehat{G} \mid{ }^{t} g\left[\begin{array}{cc}
1 & 0 \\
0 & -\ell
\end{array}\right] g= \pm\left[\begin{array}{cc}
1 & 0 \\
0 & -\ell
\end{array}\right]\right\}
$$

Then

$$
G_{n, \ell}=S_{n, \ell} \rtimes\left\langle\left[\begin{array}{cc}
1 & 0 \\
0 & -1
\end{array}\right]\right\rangle .
$$

Proof. The proofs of (1) and (2) are straightforward.

For (3), assume that $x, y \in \mathbb{R}$ with $x^{2}, y^{2}, x y / \sqrt{n} \in \mathbb{Q}$ satisfy $y+x \sqrt{\ell}=1$. Then $\left(y^{2}+\ell x^{2}\right)+$ $2(x y / \sqrt{n}) \sqrt{\ell n}=(y+x \sqrt{\ell})^{2}=1$. Our assumptions yields $y^{2}+\ell x^{2}=1$ and $x y=0$. If $x=0$, then $y= \pm 1$. If $y=0$, then $\ell=1$ and $x=1$. Hence the conclusion holds.

(4) follows from direct computations.

Then the Dirichlet unit theorem yields the following corollary.

Corollary 7.5. If $\ell>1$, then $S_{n, \ell} \cong \mathbb{Z} \oplus \mathbb{Z} / 2 \mathbb{Z}$.

Proof. Let $p_{1}, p_{2}, \ldots, p_{m}$ be the prime divisors of $\ell n$ and $\mathfrak{o}$ the ring of algebraic integers in $\mathbb{Q}\left(\sqrt{p_{1}}, \sqrt{p_{2}}, \ldots, \sqrt{p_{m}}\right)$. By Dirichlet unit theorem $\mathfrak{o}^{\times}$is a finitely generated abelian group whose torsion subgroup is $\{ \pm 1\}$. Hence $\phi\left(S_{n, \ell}\right)$ is a finitely generated abelian group whose torsion subgroup is $\{ \pm 1\}$. For $A \in S_{n, \ell}$, we have $\phi\left(A^{2}\right) \in \mathbb{Z}[\sqrt{\ell n}]$. Since $\mathbb{Z}[\sqrt{n \ell}]^{\times} \cong \mathbb{Z} \oplus \mathbb{Z} / 2 \mathbb{Z}$, we get $S_{n, \ell} \cong \mathbb{Z} \oplus \mathbb{Z} / 2 \mathbb{Z}$.

Remark 7.6. If $n=1$ and $\ell>1$, then $S_{1, \ell}$ is the group of units of $\mathbb{Z}[\sqrt{\ell}]$. Moreover if $\ell$ is square free and $\ell \equiv 2,3(\bmod 4)$, then since $\mathbb{Z}[\sqrt{\ell}]$ is the ring of the integers of $\mathbb{Q}[\sqrt{\ell}]$, a generator of $S_{1, \ell}$ becomes a fundamental unit.

Now we will construct families of simple complexes for all numerical solutions of $(1,0,-\ell)$. 
Lemma 7.7. For two positive isotropic Mukai vectors $w_{0}, w_{1}$ on the fixed abelian surface $X$, the condition

$$
(1,0,-\ell)= \pm\left(\ell w_{0}-w_{1}\right), \quad\left\langle w_{0}, w_{1}\right\rangle=-1
$$

is equivalent to

$$
w_{0}=\left(p^{2},-\frac{p q}{\sqrt{n}} H, q^{2}\right), w_{1}=\left(q^{2},-\frac{\ell p q}{\sqrt{n}} H, \ell^{2} p^{2}\right), \quad P(p, q) \in S_{n, \ell} .
$$

Proof. If there are isotropic Mukai vectors with the first condition, then we can write them as $w_{0}=(r, d H,(r \ell \pm 1))$ and $w_{1}=\left(r \ell \mp 1, d \ell H, r \ell^{2}\right)$, where $d^{2}\left(H^{2}\right)=2 r(r \ell \mp 1)$. We set $p:=\sqrt{r}$ and $q:=\sqrt{r \ell \mp 1}$. Then $w_{0}=\left(p^{2},-\frac{p q}{\sqrt{n}} H, q^{2}\right), w_{1}=\left(q^{2},-\frac{\ell p q}{\sqrt{n}} H, \ell^{2} p^{2}\right)$ and $\ell p^{2}-q^{2}= \pm 1$. Thus $P(p, q) \in S_{n, \ell}$. The converse is obvious.

Corollary 7.8. Recall the action · of $\mathrm{GL}(2, \mathbb{R})$ given in (6.14). By the correspondence

$$
g \in G_{n, \ell} \mapsto\left(w_{0}, w_{1}\right), w_{0}=(0,0,1) \cdot g, w_{1}=(1,0,0) \cdot g
$$

we have a bijective correspondence:

$$
G_{n, \ell} /\left\langle \pm\left[\begin{array}{cc}
1 & 0 \\
0 & -1
\end{array}\right]\right\rangle \cong S_{n, \ell} /\{ \pm 1\} \longleftrightarrow\left\{\left(w_{0}, w_{1}\right) \mid \begin{array}{r}
\left\langle w_{0}, w_{1}\right\rangle=-1,\left\langle w_{0}^{2}\right\rangle=\left\langle w_{1}^{2}\right\rangle=0 \\
w_{0}, w_{1}>0,(1,0,-\ell)= \pm\left(\ell w_{0}-w_{1}\right)
\end{array}\right\}
$$

Definition 7.9. Assume that $\ell>1$. Let $z:=q+\sqrt{\ell} p(p, q>0)$ be the generator of $S_{n, \ell} /\{ \pm 1\}$. We set $\epsilon:=q^{2}-\ell p^{2} \in\{ \pm 1\}$. For $m \in \mathbb{Z}$, we set

$$
\left[\begin{array}{cc}
q & \ell p \\
p & q
\end{array}\right]^{m}=\left[\begin{array}{cc}
b_{m} & \ell a_{m} \\
a_{m} & b_{m}
\end{array}\right]
$$

By the definition we have

$$
\left(a_{0}, b_{0}\right)=(0,1), \quad\left(a_{-m}, b_{-m}\right)=\epsilon^{m}\left(-a_{m}, b_{m}\right), m \in \mathbb{Z}_{>0}
$$

and

$$
S_{n, \ell}=\left\{ \pm\left[\begin{array}{cc}
b_{m} & \ell a_{m} \\
a_{m} & b_{m}
\end{array}\right] \mid m \in \mathbb{Z}\right\}
$$

Next we consider the right action of $\operatorname{GL}(2, \mathbb{R})$ on $\mathbb{R}^{2}$

$$
(x, y) \mapsto(x, y) X, \quad X \in \mathrm{GL}(2, \mathbb{R}) .
$$

Then the quadratic map

$$
\begin{aligned}
\mathbb{R}^{2} & \rightarrow \operatorname{Sym}_{2}(\mathbb{R}) \\
(x, y) & \mapsto\left[\begin{array}{l}
x \\
y
\end{array}\right]\left[\begin{array}{ll}
x & y
\end{array}\right]
\end{aligned}
$$

is $\operatorname{GL}(2, \mathbb{R})$-equivariant.

Using this action, we have the next descriptions of the topological invariants of fine moduli spaces $M_{X}^{H}(v)$ of dimension 2 .

$$
\begin{aligned}
&\left\{\begin{array}{r}
v \in H^{\mathrm{ev}}(X, \mathbb{Z})_{\mathrm{alg}},\left\langle v^{2}\right\rangle=0, v>0, \\
\langle w, v\rangle=-1, \exists w \in H^{\mathrm{ev}}(X, \mathbb{Z})_{\mathrm{alg}}
\end{array}\right\} \\
& \stackrel{\varphi_{1}}{\longleftrightarrow}\left\{\begin{array}{l|l}
(a \sqrt{r}, b \sqrt{s}) \in \mathbb{R}^{2} \mid \begin{array}{c}
a, b \in \mathbb{Z}, r, s \in \mathbb{Z}>0 \\
r s=n, \operatorname{gcd}(a r, b s)=1
\end{array}
\end{array}\right\} /\{ \pm 1\}=\{(0,1) X \mid X \in G\} /\{ \pm 1\} \\
& \stackrel{\varphi_{2}}{\longleftrightarrow}\left\{\frac{b \sqrt{n}}{a r} \in \mathbb{P}^{1}(\mathbb{R})=\mathbb{R} \cup\{\infty\} \mid r s=n, \operatorname{gcd}(a r, b s)=1\right\},
\end{aligned}
$$


where we used the correspondences

$$
v=\left(a^{2} r, a b H, b^{2} s\right) \stackrel{\varphi_{1}}{\longleftrightarrow} \pm(a \sqrt{r}, b \sqrt{s}) \stackrel{\varphi_{2}}{\longleftrightarrow} \frac{\mu(v)}{\sqrt{n}}=\frac{b \sqrt{n}}{a r} .
$$

These correspondences are $\widehat{G}$-equivariant under the action (7.2).

Lemma 7.7, (7.1) and (7.3) imply the following one to one correspondence:

$$
\begin{aligned}
\left.\left\{v_{1}, v_{2}\right\} \mid \begin{array}{l}
\text { There is a numerical solution } \\
\left(v_{1}, v_{2}, \ell_{1}, \ell_{2}\right) \text { of }(1,0,-\ell)
\end{array}\right\} & \longleftrightarrow\left\{\left\{\frac{b_{m}}{a_{m}}, \frac{\ell a_{m}}{b_{m}}\right\} \subset \mathbb{P}^{1}(\mathbb{R}) \mid m \in \mathbb{Z}\right\} \\
\left\{v_{1}, v_{2}\right\} & \longleftrightarrow\left\{\frac{\mu\left(v_{1}\right)}{\sqrt{n}}, \frac{\mu\left(v_{2}\right)}{\sqrt{n}}\right\},
\end{aligned}
$$

where $\left(\ell_{i}, \ell_{j}\right)=(\ell, 1)$ if and only if $\left(\frac{\mu\left(v_{i}\right)}{\sqrt{n}}, \frac{\mu\left(v_{j}\right)}{\sqrt{n}}\right)=\left(\frac{b_{m}}{a_{m}}, \frac{\ell a_{m}}{b_{m}}\right)$.

Definition 7.10. For the numerical solution $\left(v_{1}, v_{2}, \ell_{1}, \ell_{2}\right)$ corresponding to $\left\{\frac{b_{-m}}{a_{-m}}, \frac{\ell a_{-m}}{b_{-m}}\right\}=$ $\left\{-\frac{b_{m}}{a_{m}},-\frac{\ell a_{m}}{b_{m}}\right\}, \mathfrak{M}_{m}^{ \pm}$denotes the moduli space $\mathfrak{M}^{ \pm}\left(v_{1}, v_{2}, \ell_{1}, \ell_{2}\right) . \mathfrak{M}_{m}^{ \pm}[-1]$ denotes the moduli space of $V^{\bullet}[-1], V^{\bullet} \in \mathfrak{M}_{m}^{ \pm}$.

In this section, we will explicitly construct families $\left\{F_{m}^{\bullet}\right\}_{m \in \mathbb{Z}}$ of complexes associated to all $\left\{-\frac{b_{m}}{a_{m}},-\frac{\ell a_{m}}{b_{m}}\right\}, m \in \mathbb{Z}$.

Proposition 7.11. We have families of complexes $\left\{F_{m}^{\bullet}\right\}_{m \in \mathbb{Z}}$ such that

$$
\begin{cases}F_{m}^{\bullet} \in \mathfrak{M}_{m+1}^{+} \cap \mathfrak{M}_{m}^{-} & m>0, \\ F_{0}^{\bullet} \in \mathfrak{M}_{1}^{+} \cap \mathfrak{M}_{0}^{+}[-1], & \\ F_{m}^{\bullet} \in \mathfrak{M}_{m}^{+}[-1] \cap \mathfrak{M}_{m+1}^{-}[-1] & m<0,\end{cases}
$$

and a sequence of isomorphisms:

$$
\begin{aligned}
& \begin{array}{ccccc}
\ldots \stackrel{\Psi}{\rightarrow} & \mathfrak{M}_{0}^{-}[-1] \cap \mathfrak{M}_{-1}^{+}[-1] & \stackrel{\Psi}{\rightarrow} & \mathfrak{M}_{1}^{+} \cap \mathfrak{M}_{0}^{+}[-1] & \stackrel{\Psi}{\rightarrow} \cdots \\
\ldots \mapsto & F_{-1}^{\bullet} & \mapsto & F_{0}^{\bullet} & \mapsto \cdots
\end{array} \\
& \begin{array}{lcccc}
\ldots \stackrel{\Psi}{\rightarrow} & \mathfrak{M}_{m}^{+} \cap \mathfrak{M}_{m-1}^{-} & \stackrel{\Psi}{\rightarrow} & \mathfrak{M}_{m+1}^{+} \cap \mathfrak{M}_{m}^{-} & \stackrel{\Psi}{\rightarrow} \cdots \\
\cdots \mapsto & F_{m-1}^{\bullet} & \mapsto & F_{m}^{\bullet} & \mapsto \cdots
\end{array}
\end{aligned}
$$

where $\Psi$ is the isomorphism in Proposition 4.11.

We will call the complex $F_{m}^{\bullet}$ the tame complex. Its construction and the proof of Proposition 7.11 will be given in the following subsections.

7.2. Construction of the tame complex: case I. Under the notation of the last subsection, we assume $\epsilon=q^{2}-\ell p^{2}=-1$. In this case, the algebraic integers $a_{m}, b_{m}$ in Definition 7.9 satisfies the following relations.

$$
\frac{b_{2 k-1}}{a_{2 k-1}}<\frac{\ell a_{2 k}}{b_{2 k}}<\frac{b_{2 k-1}}{a_{2 k-1}}<\sqrt{\ell}<\frac{\ell a_{2 k+1}}{b_{2 k+1}}<\frac{b_{2 k}}{a_{2 k}}<\frac{\ell a_{2 k-1}}{b_{2 k-1}}\left(k \in \mathbb{Z}_{>0}\right), \quad \lim _{k \rightarrow \infty} \frac{b_{k}}{a_{k}}=\sqrt{\ell} .
$$

We regard $\left(a_{m}: b_{m}\right)$ and $\left(b_{m}: \ell a_{m}\right), m \in \mathbb{Z}$ as elements of $\mathbb{P}^{1}(\mathbb{R})$. Then the inhomogeneous coordinates of these points give a sequence

$$
\begin{aligned}
-\infty=\frac{b_{0}}{a_{0}} & <-\frac{\ell p}{q}=\frac{\ell a_{-1}}{b_{-1}}<\frac{b_{-2}}{a_{-2}}<\cdots<-\sqrt{\ell}<\cdots<\frac{\ell a_{-2}}{b_{-2}}<\frac{b_{-1}}{a_{-1}}=-\frac{q}{p} \\
& <\frac{\ell a_{0}}{b_{0}}=0<\frac{b_{1}}{a_{1}}=\frac{q}{p}<\frac{\ell a_{2}}{b_{2}}<\cdots<\sqrt{\ell}<\cdots<\frac{b_{2}}{a_{2}}<\frac{\ell a_{1}}{b_{1}}=\frac{\ell p}{q}<\frac{b_{0}}{a_{0}}=\infty
\end{aligned}
$$

where we write the inhomogeneous coordinate of $(0: 1)$ as $\infty$ or $-\infty$. 
In our case $\epsilon=-1$, we have the cokernel presentation

$$
0 \rightarrow E_{1} \rightarrow E_{2} \rightarrow F \rightarrow 0
$$

for a general $F \in M_{X}^{H}(1,0,-\ell)$. By Lemma 7.7, we can write $v\left(E_{1}\right)=\left(q^{2},-\frac{\ell p q}{\sqrt{n}} H, \ell^{2} p^{2}\right)$ and $v\left(E_{2}\right)=\ell\left(p^{2},-\frac{p q}{\sqrt{n}} H, q^{2}\right)$. Now we set

$$
X_{1}:=X, \quad X_{0}:=M_{X}^{H}\left(p^{2},-\frac{p q}{\sqrt{n}} H, q^{2}\right) .
$$

Since

$$
\left[\begin{array}{cc}
q & -\ell p \\
p & -q
\end{array}\right] \in G
$$

there is a universal family $\mathbf{E}$ on $X_{0} \times X_{1}$ such that the FMT $\Phi_{X_{0} \rightarrow X_{1}}^{\mathbf{E}}: \mathbf{D}\left(X_{0}\right) \rightarrow \mathbf{D}\left(X_{1}\right)$ satisfies

$$
\theta\left(\Phi_{X_{0} \rightarrow X_{1}}^{\mathbf{E}}\right)=\left[\begin{array}{cc}
q & -\ell p \\
p & -q
\end{array}\right] .
$$

Then the cokernel presentation (7.5) is rewritten as

$$
0 \rightarrow \Phi_{X_{0} \rightarrow X_{1}}^{\mathbf{E}}\left(B_{0}\right) \rightarrow \Phi_{X_{0} \rightarrow X_{1}}^{\mathbf{E}}\left(A_{0}\right) \rightarrow F \rightarrow 0
$$

where $B_{0} \in M_{X_{0}}^{\widehat{H}}(1,0,0)$ and $A_{0} \in M_{X_{0}}^{\widehat{H}}(0,0, \ell)$. Here $\widehat{H}$ is the ample generator of $\operatorname{NS}\left(X_{0}\right)$. On the other hand, every $F \in M_{X}^{H}(1,0,-\ell)$ has the kernel presentation

$$
0 \rightarrow F \rightarrow B_{1} \rightarrow A_{1} \rightarrow 0
$$

with $B_{1} \in \widehat{X}=M_{X}^{H}(1,0,0)$ and $A_{1} \in M_{X}^{H}(0,0, \ell)$.

For $i \in \mathbb{Z}$, we set

$$
X_{i}:=X_{i \bmod 2}, \quad A_{i}:=A_{i \bmod 2}, \quad B_{i}:=B_{i \bmod 2} .
$$

Definition 7.12. We define $\mathbf{E}_{i, m} \in \mathbf{D}\left(X_{i} \times X_{i+m}\right), m>0$ inductively by

$$
\mathbf{E}_{i, 0}\left[1-(-1)^{i}\right]=\mathcal{O}_{\Delta}, \quad \Phi_{X_{i+m-1} \rightarrow X_{i+m}}^{\mathbf{E}\left[1-(-1)^{i+m-1}\right]} \Phi_{X_{i} \rightarrow X_{i+m-1}}^{\mathbf{E}_{i, m-1}\left[1-(-1)^{i}\right]}=\Phi_{X_{i} \rightarrow X_{i+m}}^{\mathbf{E}_{i, m}\left[1-(-1)^{i}\right]} \quad(m>0) .
$$

Here the symbol $\Delta$ denotes the diagonal of $X_{i} \times X_{i}$.

For $\mathbf{E}_{i, m} \in \operatorname{Coh}\left(X_{i} \times X_{i+m}\right)$ and the isomorphism

$$
\xi: X_{i} \times X_{i+m} \rightarrow X_{i+m} \times X_{i}, \quad\left(x_{i}, x_{i+m}\right) \mapsto\left(x_{i+m}, x_{i}\right),
$$

we also denote $\xi_{*}\left(\mathbf{E}_{i, m}\right) \in \operatorname{Coh}\left(X_{i+m} \times X_{i}\right)$ by $\mathbf{E}_{i, m}$.

Lemma 7.13.

$$
\begin{aligned}
\Phi_{X_{i} \rightarrow X_{i+m}}^{\mathbf{E}_{i, m}\left[1-(-1)^{i}\right]}=\Phi_{X_{i+m-1} \rightarrow X_{i+m}}^{\mathbf{E}\left[1-(-1)^{i+m-1}\right]} \cdots \Phi_{X_{i+1} \rightarrow X_{i+2}}^{\mathbf{E}\left[1-(-1)^{i+1}\right]} \Phi_{X_{i} \rightarrow X_{i+1}}^{\mathbf{E}\left[1-(-1)^{i}\right]}, \quad(m>0), & \\
\Phi_{X_{i} \rightarrow X_{i+m}}^{\mathbf{E}_{i+m, m}^{\vee}\left[1+(-1)^{i+m}\right]} & =\left(\Phi_{X_{i+m} \rightarrow X_{i+2 m}}^{\mathbf{E}_{i+m, m}\left[1-(-1)^{i+m}\right]}\right)^{-1} \\
& =\Phi_{X_{i+m-1} \rightarrow X_{i+m}}^{\mathbf{E}^{\vee}\left[1+(-1)^{i+m}\right]} \cdots \Phi_{X_{i+1} \rightarrow X_{i+2}}^{\mathbf{E}^{\vee}\left[1+(-1)^{i+2}\right]} \Phi_{X_{i} \rightarrow X_{i+1}}^{\mathbf{E}^{\vee}\left[1+(-1)^{i+1}\right]}, \quad(m>0) .
\end{aligned}
$$

Under the identification $\xi: X_{i} \times X_{i+m} \rightarrow X_{i+m} \times X_{i}$, we have

$$
\mathbf{E}_{i+m, m}=\mathbf{E}_{i, m} .
$$


Proof. (7.8) is derived from Definition 7.12. The claim (7.9) follows from the following computation.

$$
\begin{aligned}
\Phi_{X_{i} \rightarrow X_{i+m}}^{\mathbf{E}_{i+m, m}^{\vee}\left[1+(-1)^{i+m}\right]} & =\left(\Phi_{X_{i+m} \rightarrow X_{i+2 m}}^{\mathbf{E}_{i+m}\left[1-(-1)^{i+m}\right]}\right)^{-1} \\
& =\left(\Phi_{X_{i+2 m-1} \rightarrow X_{i+2 m}}^{\mathbf{E}\left[1-(-1)^{i+2 m-1}\right]} \Phi_{X_{i+2 m-2} \rightarrow X_{i+2 m-1}}^{\mathbf{E}\left[1-(-1)^{i+2 m-2}\right]} \cdots \Phi_{X_{i+m} \rightarrow X_{i+m+1}}^{\mathbf{E}[1-(-1) i+m}\right)^{-1} \\
& =\Phi_{X_{i+m+1} \rightarrow X_{i+m}}^{\mathbf{E}^{\vee}\left[1+(-1)^{i+m}\right]} \cdots \Phi_{X_{i+2 m-1} \rightarrow X_{i+2 m-2}}^{\mathbf{E}^{\vee}\left[1+(-1)^{i+2 m-2}\right]} \Phi_{X_{i+2 m} \rightarrow X_{i+2 m-1}}^{\mathbf{E}^{\vee}\left[1+(-1)^{i+2 m-1}\right]} \\
& =\Phi_{X_{i+m-1} \rightarrow X_{i+m}}^{\mathbf{E}^{\vee}\left[1+(-1)^{i+m}\right]} \cdots \Phi_{\left.X_{i+1} \rightarrow X_{i+2}\right]}^{\mathbf{E}^{\vee}\left[1+(-1)^{i+2}\right]} \Phi_{X_{i} \rightarrow X_{i+1}}^{\mathbf{E}^{\vee}\left[1+(-1)^{i+1}\right]} .
\end{aligned}
$$

We prove the last claim. By using Lemma 2.8, we see that

$$
\begin{aligned}
\Phi_{X_{i} \rightarrow X_{i+m}}^{\mathbf{E}_{i, m}^{\vee}\left[1+(-1)^{i}\right]} & =\mathcal{D}_{X_{i+m}} \Phi_{X_{i} \rightarrow X_{i+m}}^{\mathbf{E}_{i, m}\left[1-(-1)^{i}\right]} \mathcal{D}_{X_{i}} \\
& =\mathcal{D}_{X_{i+m}} \Phi_{X_{i+m-1} \rightarrow X_{i+m}}^{\mathbf{E}\left[1-(-1)^{i+m-1}\right]} \cdots \Phi_{X_{i+1} \rightarrow X_{i+2}}^{\mathbf{E}\left[1-(-1)^{i+1}\right]} \Phi_{X_{i} \rightarrow X_{i+1}}^{\mathbf{E}\left[1-(-1)^{i}\right]} \mathcal{D}_{X_{i}} \\
& =\Phi_{X_{i+m-1} \rightarrow X_{i+m}}^{\mathbf{E}^{\vee}\left[1+(-1)^{i+m-1}\right]} \cdots \Phi_{X_{i+1} \rightarrow X_{i+2}}^{\mathbf{E}^{\vee}\left[1+(-1)^{i+1}\right]} \Phi_{X_{i} \rightarrow X_{i+1}}^{\mathbf{E}^{\vee}\left[1+(-1)^{i}\right]} .
\end{aligned}
$$

Hence $\Phi_{X_{i} \rightarrow X_{i+m}}^{\mathbf{E}_{i+m, m}^{\vee}}=\Phi_{X_{i} \rightarrow X_{i+m}}^{\mathbf{E}_{i, m}}$, which implies (17.10).

If $m$ is even, then

$$
\Phi_{X_{i} \rightarrow X_{i}}^{\mathbf{E}_{i, m}^{\vee}\left[1+(-1)^{i}\right]}=\left(\Phi_{X_{i} \rightarrow X_{i}}^{\mathbf{E}_{i, m}\left[1-(-1)^{i}\right]}\right)^{-1}=\left(\Phi_{X_{i} \rightarrow X_{i}}^{\mathbf{E}_{i, 2}\left[1-(-1)^{i}\right]}\right)^{-m / 2}
$$

If $m$ is odd, then

$$
\begin{aligned}
\Phi_{X_{i} \rightarrow X_{i+1}}^{\mathbf{E}_{i, m}^{\vee}\left[1+(-1)^{i+1}\right]} & =\Phi_{X_{i} \rightarrow X_{i+1}}^{\mathbf{E}_{i+1, m}^{\vee}\left[1+(-1)^{i+1}\right]}=\left(\Phi_{X_{i+1} \rightarrow X_{i}}^{\mathbf{E}_{i+1, m}\left[1-(-1)^{i+1}\right]}\right)^{-1} \\
& =\left(\Phi_{X_{i+1} \rightarrow X_{i+1}}^{\mathbf{E}_{i+1,2}\left[1-(-1)^{i+1}\right]}\right)^{-(m-1) / 2}\left(\Phi_{X_{i+1} \rightarrow X_{i}}^{\mathbf{E}\left[1-(-1)^{i+1}\right]}\right)^{-1} .
\end{aligned}
$$

The next lemma compute the cohomological action of $\mathbf{E}_{i, m}$.

Lemma 7.14. (1) For $m \in \mathbb{Z}_{\geq 0}$ we have

$$
\begin{gathered}
\theta\left(\Phi_{X_{0} \rightarrow X_{m}}^{\mathbf{E}_{0, m}}\right)= \begin{cases} & \pm\left[\begin{array}{cc}
q & \ell p \\
p & q
\end{array}\right]^{m}= \pm\left[\begin{array}{cc}
b_{m} & \ell a_{m} \\
a_{m} & b_{m}
\end{array}\right] \quad m \in 2 \mathbb{Z}_{\geq 0}, \\
\pm\left[\begin{array}{cc}
q & \ell p \\
p & q
\end{array}\right]^{m}\left[\begin{array}{cc}
1 & 0 \\
0 & -1
\end{array}\right]= \pm\left[\begin{array}{cc}
b_{m} & -\ell a_{m} \\
a_{m} & -b_{m}
\end{array}\right] & m \in 2 \mathbb{Z}_{\geq 0}+1,\end{cases} \\
\theta\left(\Phi_{X_{1} \rightarrow X_{1+m}}^{\mathbf{E}_{1, m}[2]}\right)= \begin{cases} \pm\left[\begin{array}{cc}
-q & \ell p \\
p & -q
\end{array}\right]^{m}= \pm\left[\begin{array}{cc}
-b_{m} & -\ell a_{m} \\
a_{m} & -b_{m}
\end{array}\right] & m \in 2 \mathbb{Z}_{\geq 0}, \\
\pm\left[\begin{array}{cc}
-q & \ell p \\
p & -q
\end{array}\right]^{m}\left[\begin{array}{cc}
1 & 0 \\
0 & -1
\end{array}\right]= \pm\left[\begin{array}{cc}
-b_{m} & -\ell a_{m} \\
a_{m} & b_{m}
\end{array}\right] & m \in 2 \mathbb{Z}_{\geq 0}+1 .\end{cases}
\end{gathered}
$$

In these equations, the signs in front of the matrices are irrelevant, since the images of $\theta$ in $\operatorname{PSL}(2, \mathbb{R})$.

(2) $\mathbf{E}_{i, m}$ is a locally free sheaf for $m \in \mathbb{Z}_{>0}$.

Proof. (1) By Lemma 6.18 we have

$$
\theta\left(\Phi_{X_{1} \rightarrow X_{0}}^{\mathbf{E}}\right)= \pm\left[\begin{array}{cc}
-q & -\ell p \\
p & q
\end{array}\right]
$$

Hence we find that

$$
\theta\left(\Phi_{X_{1} \rightarrow X_{0}}^{\mathbf{E}} \Phi_{X_{0} \rightarrow X_{1}}^{\mathbf{E}}\right)=\theta\left(\Phi_{X_{0} \rightarrow X_{1}}^{\mathbf{E}}\right) \theta\left(\Phi_{X_{1} \rightarrow X_{0}}^{\mathbf{E}}\right)= \pm\left[\begin{array}{cc}
q & \ell p \\
p & q
\end{array}\right]^{2},
$$




$$
\theta\left(\Phi_{X_{0} \rightarrow X_{1}}^{\mathbf{E}} \Phi_{X_{1} \rightarrow X_{0}}^{\mathbf{E}}\right)= \pm\left[\begin{array}{cc}
q & -\ell p \\
-p & q
\end{array}\right]^{2}
$$

From these relations we obtain the conclusion.

(2) Assume that $\mathbf{E}_{i, m-1}$ is a locally free sheaf. By Lemma 7.14 (1), we have

$$
\begin{array}{ll}
\mu\left(\left.\mathbf{E}_{i, m}\right|_{X_{i} \times\left\{x_{i+m}\right\}}\right) & =(-1)^{i} \frac{b_{m}}{a_{m}} \sqrt{n}, \\
\mu\left(\left.\mathbf{E}_{i, m-1}\right|_{\left\{x_{i}\right\} \times X_{i+m-1}}\right) & =(-1)^{i+m-1} \frac{b_{m-1}}{a_{m-1}} \sqrt{n}, \\
\mu\left(\left.\mathbf{E}\right|_{X_{i+m-1} \times\left\{x_{i+m}\right\}}\right) & =(-1)^{i+m-1} \frac{q}{p} \sqrt{n},
\end{array}
$$

Here $x_{i}$ is a closed point of $X_{i}$. Propositions 2.14 (1) and (7.4) yield that if $i+m-1$ is even then $\Phi_{X_{i} \rightarrow X_{i+m}}^{\mathbf{E}_{i, m}}\left(\mathbb{C}_{x_{i}}\right)=\Phi_{X_{i+m-1} \rightarrow X_{i+m}}^{\mathbf{E}} \Phi_{X_{i} \rightarrow X_{i+m-1}}^{\mathbf{E}_{i, m-1}}\left(\mathbb{C}_{x_{i}}\right)$ is a locally free sheaf. In the same manner if $i+m-1$ is odd, then $\Phi_{X_{i} \rightarrow X_{i+m}}^{\mathbf{E}_{i, m}}\left(\mathbb{C}_{x_{i}}\right)=\Phi_{X_{i+m-1} \rightarrow X_{i+m}}^{\mathbf{E}[2]} \Phi_{X_{i} \rightarrow X_{i+m-1}}^{\mathbf{E}_{i, m-1}}\left(\mathbb{C}_{x_{i}}\right)$ is a locally free sheaf. Therefore $\mathbf{E}_{i, m}$ is also a locally free sheaf. Thus the conclusion holds.

The next weak index theorem is the fundamental result for our computation of FMTs.

Lemma 7.15. For $m \geq 0$, we have

$$
\begin{aligned}
& \Phi_{X_{0} \rightarrow X_{m}}^{\mathbf{E}_{0, m}}\left(A_{0}\right), \quad \Phi_{X_{0} \rightarrow X_{m}}^{\mathbf{E}_{0, m}}\left(A_{0}^{\vee}[2]\right) \quad \in \operatorname{Coh}\left(X_{m}\right), \\
& \Phi_{X_{0} \rightarrow X_{m}}^{\mathbf{E}_{0, m}}\left(B_{0}\right), \quad \Phi_{X_{0} \rightarrow X_{m}}^{\mathbf{E}_{0, m}}\left(B_{0}^{\vee}\right), \quad \Phi_{X_{0} \rightarrow X_{m}}^{\mathbf{E}_{0, m}^{\vee}[2]}\left(B_{0}\right), \quad \Phi_{X_{0} \rightarrow X_{m}}^{\mathbf{E}_{0, m}^{\vee}[2]}\left(B_{0}^{\vee}\right) \quad \in \operatorname{Coh}\left(X_{m}\right), \\
& \Phi_{X_{1} \rightarrow X_{1+m}}^{\mathbf{E}_{1, m}}\left(A_{1}\right), \quad \Phi_{X_{1} \rightarrow X_{1+m}}^{\mathbf{E}_{1, m}}\left(A_{1}^{\vee}[2]\right) \quad \in \operatorname{Coh}\left(X_{1+m}\right), \\
& \Phi_{X_{1} \rightarrow X_{1+m}}^{\mathbf{E}_{1, m}[2]}\left(B_{1}\right), \quad \Phi_{X_{1} \rightarrow X_{1+m}}^{\mathbf{E}_{1, m}[2]}\left(B_{1}^{\vee}\right), \quad \Phi_{X_{1} \rightarrow X_{1+m}}^{\mathbf{E}_{1, m}^{\vee}}\left(B_{1}\right), \quad \Phi_{X_{1} \rightarrow X_{1+m}}^{\mathbf{E}_{1, m}^{\vee}}\left(B_{1}^{\vee}\right) \quad \in \operatorname{Coh}\left(X_{1+m}\right) .
\end{aligned}
$$

Proof. We may assume that $m>0$. Since $A_{i}, A_{i}^{\vee}[2], i=0,1$ are 0 -dimensional sheaves, the claims hold for $A_{i}, A_{i}^{\vee}[2], i=0,1$. Since $B_{i}, B_{i}^{\vee}, i=0,1$ are line bundles of degree 0 , $\mu\left(\left.\mathbf{E}_{j, m}\right|_{X_{j} \times\left\{x_{j+m}\right\}}\right)=(-1)^{j}\left(b_{m} / a_{m}\right) \sqrt{n}$ implies that $\mathrm{WIT}_{0}$ (resp. $\mathrm{WIT}_{2}$ ) holds for $B_{0}, B_{0}^{\vee}$ with respect to $\Phi_{X_{0} \rightarrow X_{m}}^{\mathbf{E}_{0, m}}$ (resp. $\Phi_{X_{0} \rightarrow X_{m}}^{\mathbf{E}_{0, m}^{\vee}}$ ) and $\mathrm{WIT}_{2}$ (resp. WIT ${ }_{0}$ ) holds for $B_{1}, B_{1}^{\vee}$ with respect to $\Phi_{X_{1} \rightarrow X_{1+m}}^{\mathbf{E}_{1, m}}$ (resp. $\Phi_{X_{1} \rightarrow X_{1+m}}^{\mathbf{E}_{1, m}^{\vee}}$ ).

From (7.6) and (7.7) we have

$$
0 \rightarrow \Phi_{X_{0} \rightarrow X_{1}}^{\mathbf{E}}\left(B_{0}\right) \rightarrow \Phi_{X_{0} \rightarrow X_{1}}^{\mathbf{E}}\left(A_{0}\right) \rightarrow B_{1} \rightarrow A_{1} \rightarrow 0 .
$$

Applying $\Phi_{X_{1} \rightarrow X_{0}}^{\mathbf{E}^{\vee}[2]}$ to this sequence, we have

$$
0 \rightarrow \Phi_{X_{1} \rightarrow X_{0}}^{\mathbf{E}^{\vee}}\left(B_{1}\right) \rightarrow \Phi_{X_{0} \rightarrow X_{1}}^{\mathbf{E}^{\vee}}\left(A_{1}\right) \rightarrow B_{0} \rightarrow A_{0} \rightarrow 0 .
$$

For later use, we prepare the next lemma.

\section{Lemma 7.16.}

$$
\begin{array}{ll}
\Phi_{X_{0} \rightarrow X_{1}}^{\mathbf{E}_{0,2 k+1}} \mathcal{D}_{X_{0}} \Phi_{X_{1} \rightarrow X_{0}}^{\mathbf{E}^{\vee}}=\Phi_{X_{1} \rightarrow X_{1}}^{\mathbf{E}_{1,2 k+2}[2]} \mathcal{D}_{X_{1}}, & \Phi_{X_{0} \rightarrow X_{1}}^{\mathbf{E}_{0,2 k+1}} \Phi_{X_{1} \rightarrow X_{0}}^{\mathbf{E}^{\vee}}=\Phi_{X_{1} \rightarrow X_{1}}^{\mathbf{E}_{1,2 k}}, \\
\Phi_{X_{1} \rightarrow X_{1}}^{\mathbf{E}_{1,2 k}[2]} \mathcal{D}_{X_{1}} \Phi_{X_{0} \rightarrow X_{1}}^{\mathbf{E}}=\Phi_{X_{0} \rightarrow X_{1}}^{\mathbf{E}_{0,2 k-1}[2]} \mathcal{D}_{X_{0}}, & \Phi_{X_{1} \rightarrow X_{1}}^{\mathbf{E}_{1,2 k}[2]} \Phi_{X_{0} \rightarrow X_{1}}^{\mathbf{E}}=\Phi_{X_{0} \rightarrow X_{1}}^{\mathbf{E}_{0,2 k+1}}
\end{array}
$$

Proof. Recall Lemma 2.8 and the definition of $\mathbf{E}_{i, m}$ (Definition 7.12). One can compute that

$$
\begin{aligned}
& \Phi_{X_{0} \rightarrow X_{1}}^{\mathbf{E}_{0,2 k+1}} \mathcal{D}_{X_{0}} \Phi_{X_{1} \rightarrow X_{0}}^{\mathbf{E}^{\vee}}=\Phi_{X_{0} \rightarrow X_{1}}^{\mathbf{E}_{0,2 k+1}} \Phi_{X_{1} \rightarrow X_{0}}^{\mathbf{E}[2]} \mathcal{D}_{X_{0}}=\Phi_{X_{1} \rightarrow X_{1}}^{\mathbf{E}_{1,2 k+1}[2]} \mathcal{D}_{X_{0}}, \\
& \Phi_{X_{0} \rightarrow X_{1}}^{\mathbf{E}_{0,2 k+1}} \Phi_{X_{1} \rightarrow X_{0}}^{\mathbf{E}^{\vee}}=\Phi_{X_{0} \rightarrow X_{1}}^{\mathbf{E}_{0,2 k+1}}\left(\Phi_{X_{0} \rightarrow X_{1}}^{\mathbf{E}}\right)^{-1}[-2]=\Phi_{X_{1} \rightarrow X_{1}}^{\mathbf{E}_{1,2 k}[2]}[-2], \\
& \Phi_{X_{1} \rightarrow X_{1}}^{\mathbf{E}_{1,2 k}[2]} \mathcal{D}_{X_{1}} \Phi_{X_{0} \rightarrow X_{1}}^{\mathbf{E}}=\Phi_{X_{1} \rightarrow X_{1}}^{\mathbf{E}_{1,2 k}[2]} \Phi_{X_{0} \rightarrow X_{1}}^{\mathbf{E}^{\vee}[2]} \mathcal{D}_{X_{0}}=\Phi_{X_{1} \rightarrow X_{1}}^{\mathbf{E}_{1,2 k}[2]}\left(\Phi_{X_{1} \rightarrow X_{0}}^{\mathbf{E}[2]}\right)^{-1}[2] \mathcal{D}_{X_{0}}=\Phi_{X_{0} \rightarrow X_{1}}^{\mathbf{E}_{0,2 k-1}[2]} \mathcal{D}_{X_{0}} .
\end{aligned}
$$


The last equality follows from the second one.

Definition 7.17. For

$$
F:=\operatorname{Ker}\left(B_{1} \rightarrow A_{1}\right) \cong \operatorname{Coker}\left(\Phi_{X_{0} \rightarrow X_{1}}^{\mathbf{E}}\left(B_{0}\right) \rightarrow \Phi_{X_{0} \rightarrow X_{1}}^{\mathbf{E}}\left(A_{0}\right)\right),
$$

we define the tame complex $F_{m}^{\bullet}$ by

$$
F_{m}^{\bullet}:=\left\{\begin{array}{llll}
\left(\Phi_{X_{1} \rightarrow X_{1}}^{\mathbf{E}_{1,2}[2]}\right)^{(m+1) / 2}\left(F^{\vee}\right) & =\Phi_{X_{1} \rightarrow X_{1}}^{\mathbf{E}_{1,2 k}[2]}\left(F^{\vee}\right) & m=2 k-1, & k \geq 1, \\
\left(\Phi_{X_{1} \rightarrow X_{1}}^{\mathbf{E}_{1,2}[2]}\right)^{m / 2}(F) & =\Phi_{X_{1} \rightarrow X_{1}}^{\mathbf{E}_{1,2 k}[2]}(F) & m=2 k, & k \geq 0, \\
\left(\Phi_{X_{1,2} \rightarrow X_{1}}^{\mathbf{E}_{1,2}[2]}\right)^{(m+1) / 2}\left(F^{\vee}\right) & =\Phi_{X_{1,2 k} \rightarrow X_{1}}^{\mathbf{E}^{\vee}}\left(F^{\vee}\right) & m=-2 k-1, & k \geq 0, \\
\left(\Phi_{X_{1} \rightarrow X_{1}}^{\mathbf{E}_{1,2}[2]}\right)^{m / 2}(F) & =\Phi_{X_{1} \rightarrow X_{1}}^{\mathbf{E}_{1,2 k}}(F) & m=-2 k, & k \geq 1 .
\end{array}\right.
$$

$F_{m}^{\bullet}$ satisfies $v\left(F_{m}^{\bullet}\right)=(1,0,-\ell)$. We can also express them in terms of $A_{0}, B_{0}$.

\section{Lemma 7.18.}

$$
F_{m}^{\bullet}= \begin{cases}\Phi_{X_{0} \rightarrow X_{1}}^{\mathbf{E}_{0,2 k-1}[2]}\left(\left[B_{0} \rightarrow A_{0}\right]^{\vee}\right) & m=2 k-1, \\ \Phi_{X_{0} \rightarrow X_{1}}^{\mathbf{E}_{0,2 k+1}}\left(\left[B_{0} \rightarrow A_{0}\right]\right) & m=2 k, \\ \Phi_{X_{0,2 k+1}[2]}^{\mathbf{E}_{1}^{\vee}\left(\left[B_{0} \rightarrow A_{0}\right] \vee\right)} & m=-2 k-1, \\ \Phi_{X_{0} \rightarrow X_{1}}^{\mathbf{E}_{1,2 k-1}^{\vee}}\left(\left[B_{0} \rightarrow A_{0}\right]\right) & m=-2 k .\end{cases}
$$

By Lemmas 7.15, 7.18 and Definition 7.17, we have the following description of $F_{m}^{\bullet}$, from which follows the first part of Proposition 7.11 for the case $\epsilon=-1$.

Proposition 7.19. (1) If $m=2 k, k \geq 1$, then $\Phi_{X_{0} \rightarrow X_{1}}^{\mathbf{E}_{0,2 k+1}}\left(B_{0}\right)$ and $\Phi_{X_{0} \rightarrow X_{1}}^{\mathbf{E}_{0,2 k+1}}\left(A_{0}\right)$ are locally free sheaves, and

$$
\begin{aligned}
& F_{2 k}^{\bullet}=\left[\Phi_{X_{0} \rightarrow X_{1}}^{\mathbf{E}_{0,2 k+1}}\left(B_{0}\right) \rightarrow \Phi_{X_{0} \rightarrow X_{1}}^{\mathbf{E}_{0,2 k+1}}\left(A_{0}\right)\right], \\
& H^{i}\left(F_{2 k}^{\bullet}\right)= \begin{cases}\Phi_{X_{1} \rightarrow X_{1}}^{\mathbf{E}_{1,2 k}}\left(A_{1}\right) & i=-1, \\
\Phi_{\left.X_{1,2 k} \rightarrow 2\right]}^{\mathbf{E}_{1}}\left(B_{1}\right) & i=0, \\
0 & i \neq-1,0 .\end{cases}
\end{aligned}
$$

In particular, we have an exact sequence

$(\# 2 k)$

$$
0 \rightarrow \Phi_{X_{1} \rightarrow X_{1}}^{\mathbf{E}_{1,2 k}}\left(A_{1}\right) \rightarrow \Phi_{X_{0} \rightarrow X_{1}}^{\mathbf{E}_{0,2 k+1}}\left(B_{0}\right) \rightarrow \Phi_{X_{0} \rightarrow X_{1}}^{\mathbf{E}_{0,2 k+1}}\left(A_{0}\right) \rightarrow \Phi_{X_{1} \rightarrow X_{1}}^{\mathbf{E}_{1,2 k}[2]}\left(B_{1}\right) \rightarrow 0 .
$$

The slopes of these four sheaves are

$$
-\frac{b_{2 k}}{a_{2 k}} \sqrt{n}<-\frac{\ell a_{2 k+1}}{b_{2 k+1}} \sqrt{n}<-\frac{b_{2 k+1}}{a_{2 k+1}} \sqrt{n}<-\frac{\ell a_{2 k}}{b_{2 k}} \sqrt{n} .
$$

(2) If $m=2 k+1, k \geq 0$, then $\Phi_{X_{1} \rightarrow X_{1}}^{\mathbf{E}_{1,2 k+2}[2]}\left(A_{1}^{\vee}\right)$ and $\Phi_{X_{1} \rightarrow X_{1}}^{\mathbf{E}_{1,2 k+2}[2]}\left(B_{1}^{\vee}\right)$ are locally free sheaves, and

$$
\begin{aligned}
& F_{2 k+1}^{\bullet}=\left[\Phi_{X_{1} \rightarrow X_{1}}^{\mathbf{E}_{1,2 k+2}[2]}\left(A_{1}^{\vee}\right) \rightarrow \Phi_{X_{1} \rightarrow X_{1}}^{\mathbf{E}_{1,2 k+2}[2]}\left(B_{1}^{\vee}\right)\right], \\
& H^{i}\left(F_{2 k+1}^{\bullet}\right)= \begin{cases}\Phi_{X_{0} \rightarrow X_{1}}^{\mathbf{E}_{1,2 k+1}}\left(B_{0}^{\vee}\right) & i=-1, \\
\Phi_{X_{0} \rightarrow X_{1}}^{\mathbf{E}_{1,2 k+1}}\left(A_{0}^{\vee}[2]\right) & i=0, \\
0 & i \neq-1,0 .\end{cases}
\end{aligned}
$$

In particular, we have an exact sequence

$$
\left(\#_{2 k+1}\right) \quad 0 \rightarrow \Phi_{X_{0} \rightarrow X_{1}}^{\mathbf{E}_{0,2 k+1}}\left(B_{0}^{\vee}\right) \rightarrow \Phi_{X_{1} \rightarrow X_{1}}^{\mathbf{E}_{1,2 k+2}[2]}\left(A_{1}^{\vee}\right) \rightarrow \Phi_{X_{1} \rightarrow X_{1}}^{\mathbf{E}_{1,2 k+2}[2]}\left(B_{1}^{\vee}\right) \rightarrow \Phi_{X_{0} \rightarrow X_{1}}^{\mathbf{E}_{0,2 k+1}}\left(A_{0}^{\vee}[2]\right) \rightarrow 0 .
$$


The slopes of these four sheaves are

$$
-\frac{\ell a_{2 k+1}}{b_{2 k+1}} \sqrt{n}<-\frac{b_{2 k+2}}{a_{2 k+2}} \sqrt{n}<-\frac{\ell a_{2 k+2}}{b_{2 k+2}} \sqrt{n}<-\frac{b_{2 k+1}}{a_{2 k+1}} \sqrt{n} .
$$

(3) If $m=-2 k-1, k \geq 0$, then $\Phi_{X_{0} \rightarrow X_{1}}^{\mathbf{E}_{1,2 k+1}^{\vee[2]}}\left(A_{0}^{\vee}\right), \Phi_{X_{0} \rightarrow X_{1}}^{\mathbf{E}_{1,2 k+1}[2]}\left(B_{0}^{\vee}\right)$ are locally free sheaves, and

$$
\begin{aligned}
& F_{-2 k-1}^{\bullet}[1]=\left[\Phi_{X_{0} \rightarrow X_{1}}^{\mathbf{E}_{1,2 k+1}^{\vee}[2]}\left(A_{0}^{\vee}\right) \rightarrow \Phi_{X_{0} \rightarrow X_{1}}^{\mathbf{E}_{1,2 k+1}^{\vee}[2]}\left(B_{0}^{\vee}\right)\right], \\
& H^{i}\left(F_{-2 k-1}^{\bullet}[1]\right)= \begin{cases}\Phi_{X_{1} \rightarrow X_{1}}^{\mathbf{E}_{1,2 k}}\left(B_{1}^{\vee}\right) & i=-1, \\
\Phi_{X_{1} \rightarrow X_{1}}^{\mathbf{E}_{1,2 k}}\left(A_{1}^{\vee}[2]\right) & i=0, \\
0 & i \neq-1,0 .\end{cases}
\end{aligned}
$$

In particular, we have an exact sequence

$$
\left(\#_{-2 k-1}\right) \quad 0 \rightarrow \Phi_{X_{1} \rightarrow X_{1}}^{\mathbf{E}_{1,2 k}^{\vee}}\left(B_{1}^{\vee}\right) \rightarrow \Phi_{X_{0} \rightarrow X_{1}}^{\mathbf{E}_{1,2 k+1}^{\vee}[2]}\left(A_{0}^{\vee}\right) \rightarrow \Phi_{X_{0} \rightarrow X_{1}}^{\mathbf{E}_{1,2 k+1}^{\vee}[2]}\left(B_{0}^{\vee}\right) \rightarrow \Phi_{X_{1} \rightarrow X_{1}}^{\mathbf{E}_{1,2 k}^{\vee}}\left(A_{1}^{\vee}[2]\right) \rightarrow 0 .
$$

The slopes of these four sheaves are

$$
\frac{\ell a_{2 k}}{b_{2 k}} \sqrt{n}<\frac{b_{2 k+1}}{a_{2 k+1}} \sqrt{n}<\frac{\ell a_{2 k+1}}{b_{2 k+1}} \sqrt{n}<\frac{b_{2 k}}{a_{2 k}} \sqrt{n}
$$

(4) If $m=-2 k-2, k \geq 0$, then $\Phi_{X_{1} \rightarrow X_{1}}^{\mathbf{E}_{1,2 k+2}}\left(B_{1}\right)$ and $\Phi_{X_{1} \rightarrow X_{1}}^{\mathbf{E}_{1,2 k+2}}\left(A_{1}\right)$ are locally free sheaves, and

$$
\begin{aligned}
& F_{-2 k-2}^{\bullet}=\left[\Phi_{X_{1} \rightarrow X_{1}}^{\mathbf{E}_{1,2 k+2}^{\vee}}\left(B_{1}\right) \rightarrow \Phi_{X_{1} \rightarrow X_{1}}^{\mathbf{E}_{1,2 k+2}^{\vee}}\left(A_{1}\right)\right], \\
& H^{i}\left(F_{-2 k-2}^{\bullet}[1]\right)= \begin{cases}\Phi_{X_{0} \rightarrow X_{1}}^{\bullet} A_{0}^{\vee} & i=-1, \\
\Phi_{X_{0} \rightarrow X_{1}}^{\mathbf{E}_{1,2 k+1}[2]}\left(B_{0}\right) & i=0, \\
0 & i \neq-1,0 .\end{cases}
\end{aligned}
$$

In particular, we have an exact sequence

$$
\left(\#_{-2 k-2}\right) \quad 0 \rightarrow \Phi_{X_{0} \rightarrow X_{1}}^{\mathbf{E}_{1,2 k+1}^{\vee}}\left(A_{0}\right) \rightarrow \Phi_{X_{1} \rightarrow X_{1}}^{\mathbf{E}_{1,2 k+2}^{\vee}}\left(B_{1}\right) \rightarrow \Phi_{X_{1} \rightarrow X_{1}}^{\mathbf{E}_{1,2 k+2}^{\vee}}\left(A_{1}\right) \rightarrow \Phi_{X_{0} \rightarrow X_{1}}^{\mathbf{E}_{1,2 k+1}^{\vee}[2]}\left(B_{0}\right) \rightarrow 0 .
$$

The slopes of these four sheaves are

$$
\frac{b_{2 k+1}}{a_{2 k+1}} \sqrt{n}<\frac{\ell a_{2 k+2}}{b_{2 k+2}} \sqrt{n}<\frac{b_{2 k+2}}{a_{2 k+2}} \sqrt{n}<\frac{\ell a_{2 k+1}}{b_{2 k+1}} \sqrt{n} .
$$

Now we show the second part of Proposition 7.11 for the case $\epsilon=-1$. By Definition 7.17 and Lemma 7.18 , the relations of $\left(\#_{m}\right), m \in \mathbb{Z}$ are summarized as follows.

$$
\begin{array}{lll}
F_{2 k}^{\bullet} & =\Phi_{X_{1} \rightarrow X_{1}}^{\mathbf{E}_{1,2 k}[2]} \mathcal{D}_{X_{1}}\left(\Phi_{X_{1} \rightarrow X_{1}}^{\mathbf{E}_{1,2 k}[2]}\right)^{-1}\left(F_{2 k-1}^{\bullet}\right) & k \in \mathbb{Z}_{\geq 1}, \\
F_{2 k+1}^{\bullet \bullet} & =\Phi_{X_{0} \rightarrow X_{1}}^{\mathbf{E}_{0,2 k+1}[2]} \mathcal{D}_{X_{0}}\left(\Phi_{X_{0} \rightarrow X_{1}}^{\mathbf{E}_{0,2 k+1}}\right)^{-1}\left(F_{2 k}^{\bullet}\right) & k \in \mathbb{Z}_{\geq 0}, \\
F_{-2 k-1}^{\bullet} & =\Phi_{X_{1} \rightarrow X_{1}}^{\mathbf{E}^{\vee}} \mathcal{D}_{X_{1}}\left(\Phi_{X_{1,2 k} \rightarrow X_{1}}^{\mathbf{E}^{\vee}}\right)^{-1}\left(F_{-2 k}^{\bullet}\right) & k \in \mathbb{Z}_{\geq 0}, \\
F_{-2 k-2}^{\bullet}=\Phi_{X_{0} \rightarrow X_{1}}^{\mathbf{E}_{1}^{\vee}} \mathcal{D}_{X_{0}}\left(\Phi_{X_{0} \rightarrow X_{1}}^{\mathbf{E}_{1,2 k+1}[2]}\right)^{-1}\left(F_{-2 k-1}^{\bullet}\right) & k \in \mathbb{Z}_{\geq 0} .
\end{array}
$$

These are nothing but the relations $\Psi\left(F_{m}^{\bullet}\right)=F_{m+1}^{\bullet}(m \in \mathbb{Z})$ indicated in the second statement of Proposition 7.11, Thus the proof of Proposition 7.11 is completed. 
7.3. Application of the tame complex. For a FMT $\Phi_{X_{1} \rightarrow X^{\prime}}^{\mathrm{G}}: \mathbf{D}\left(X_{1}\right) \rightarrow \mathbf{D}\left(X^{\prime}\right)$, we set $\lambda:=\mu\left(\mathbf{G}_{x^{\prime}}\right) / \sqrt{n}$. If $-\ell p / q<-\lambda<-q / p$, then $\Phi_{X_{1} \rightarrow X^{\prime}}^{\mathbf{G}}\left(F_{0}^{\bullet}\right)$ is not a sheaf for all $F_{0}^{\bullet}$.

Definition 7.20. We set

$$
\begin{array}{llll}
I_{0} & :=\left(0, \frac{b_{1}}{a_{1}}\right] \cup\left(\frac{\ell a_{1}}{b_{1}}, \infty\right), & I_{-1} & :=\left(-\infty,-\frac{\ell a_{1}}{b_{1}}\right] \cup\left(-\frac{b_{1}}{a_{1}}, 0\right], \\
I_{2 k-1} & :=\left(\frac{b_{2 k-1}}{a_{2 k-1}}, \frac{\ell a_{2 k}}{b_{2 k}}\right] \cup\left(\frac{b_{2 k}}{a_{2 k}}, \frac{\ell a_{2 k-1}}{b_{2 k-1}}\right], & I_{-2 k-1} & :=\left(-\frac{b_{2 k}}{a_{2 k}},-\frac{\ell a_{2 k+1}}{b_{2 k+1}}\right] \cup\left(-\frac{b_{2 k+1}}{a_{2 k+1}},-\frac{\ell a_{2 k}}{b_{2 k}}\right], \\
I_{2 k} & :=\left(\frac{\ell a_{2 k}}{b_{2 k}}, \frac{b_{2 k+1}}{a_{2 k+1}}\right] \cup\left(\frac{\ell a_{2 k+1}}{b_{2 k+1}}, \frac{b_{2 k}}{a_{2 k}}\right], & I_{-2 k} & :=\left(-\frac{\ell a_{2 k-1}}{b_{2 k-1}},-\frac{b_{2 k}}{a_{2 k}}\right] \cup\left(-\frac{\ell a_{2 k}}{b_{2 k}},-\frac{b_{2 k-1}}{a_{2 k-1}}\right] .
\end{array}
$$

For $I=\coprod_{i}\left(s_{i}, t_{i}\right]$, we denote $I^{*}:=\coprod_{i}\left[s_{i}, t_{i}\right)$.

By (17.4), we have decompositions $\mathbb{P}^{1}(\mathbb{R}) \backslash\{ \pm \sqrt{\ell}\}=\coprod_{m \in \mathbb{Z}} I_{m}=\coprod_{m \in \mathbb{Z}} I_{m}^{*}$.

Theorem 7.21. (1) If $\lambda \in I_{m}$, then $\Phi_{X_{1} \rightarrow X^{\prime}}^{\mathbf{G}}\left(F_{m}^{\bullet}\right)$ is a coherent sheaf.

(2) If $\lambda \in I_{m}^{*}$, then $\mathcal{D}_{X^{\prime}} \Phi_{X_{1} \rightarrow X^{\prime}}^{\mathbf{G}}\left(F_{m}^{\bullet}\right)=\Phi_{X_{1} \rightarrow X^{\prime}}^{\mathbf{G}^{\vee}[2]}\left(F_{m}^{\bullet \vee}\right)$ is a coherent sheaf.

Proof. We only prove (1). Assume that $m=2 k$. Then $-\frac{b_{2 k}}{a_{2 k}} \leq-\lambda \leq-\frac{\ell a_{2 k+1}}{b_{2 k+1}}$ or $-\frac{b_{2 k+1}}{a_{2 k+1}} \leq-\lambda \leq$ $-\frac{\ell a_{2 k}}{b_{2 k}}$. By $\left(\#_{2 k}\right)$ and Lemma 4.13, we get our claim. If $m=2 k-1$, then our claim also follows from $\left(\#_{2 k-1}\right)$ and Lemma 4.13 .

Remark 7.22. We will explain the operation $\mathcal{F}$ in Fact 2.17. For simplicity, we assume that $\lambda \in\left(\frac{\ell a_{2 k+1}}{b_{2 k+1}}, \frac{b_{2 k}}{a_{2 k}}\right]=I_{2 k} \cap(\sqrt{\ell}, \infty)$. Then we have

$$
\begin{aligned}
& \Phi_{X_{1} \rightarrow X^{\prime}}^{\mathbf{G}} \Phi_{X_{1} \rightarrow X_{1}}^{\mathbf{E}_{1,2 i}[2]}\left(A_{1}\right), \quad \Phi_{X_{1} \rightarrow X^{\prime}}^{\mathbf{G}} \Phi_{X_{1} \rightarrow X_{1}}^{\mathbf{E}_{1,2 i}[2]}\left(A_{1}^{\vee}[2]\right) \quad \in \operatorname{Coh}\left(X^{\prime}\right) \quad i \leq k, \\
& \Phi_{X_{1} \rightarrow X^{\prime}}^{\mathbf{G}} \Phi_{X_{0} \rightarrow X_{1}}^{\mathbf{E}_{0,2 i+}[2]}\left(B_{0}\right), \quad \Phi_{X_{1} \rightarrow X^{\prime}}^{\mathbf{G}} \Phi_{X_{0} \rightarrow X_{1}}^{\mathbf{E}_{0,2 i+1}[2]}\left(B_{0}^{\vee}\right) \quad \in \operatorname{Coh}\left(X^{\prime}\right) \quad i<k, \\
& \Phi_{X_{1} \rightarrow X^{\prime}}^{\mathbf{G}} \Phi_{X_{0} \rightarrow X_{1}}^{\mathbf{E}_{0,2 k+1}}\left(B_{0}\right), \quad \Phi_{X_{1} \rightarrow X^{\prime}}^{\mathbf{G}} \Phi_{X_{0} \rightarrow X_{1}}^{\mathbf{E}_{0,2 k+1}}\left(B_{0}^{\vee}\right) \quad \in \operatorname{Coh}\left(X^{\prime}\right), \\
& \Phi_{X_{1} \rightarrow X^{\prime}}^{\mathbf{G}} \Phi_{X_{0} \rightarrow X_{1}}^{\mathbf{E}_{0,2 i+1}}\left(A_{0}\right), \quad \Phi_{X_{1} \rightarrow X^{\prime}}^{\mathbf{G}} \Phi_{X_{0} \rightarrow X_{1}}^{\mathbf{E}_{0,2 i+1}}\left(A_{0}^{\vee}[2]\right) \quad \in \operatorname{Coh}\left(X^{\prime}\right) \quad 0 \leq i, \\
& \Phi_{X_{1} \rightarrow X^{\prime}}^{\mathbf{G}} \Phi_{X_{1} \rightarrow X_{1}}^{\mathbf{E}_{1,2 i}[2]}\left(B_{1}\right), \quad \Phi_{X_{1} \rightarrow X^{\prime}}^{\mathbf{G}} \Phi_{X_{1} \rightarrow X_{1}}^{\mathbf{E}_{1,2 i}[2]}\left(B_{1}^{\vee}\right) \quad \in \operatorname{Coh}\left(X^{\prime}\right) \quad 0 \leq i .
\end{aligned}
$$

By using (7.12), we have the following description of $F_{m}^{\bullet}, m=2 j, 2 j+1, j<k$.

- $\Phi_{X_{1} \rightarrow X^{\prime}}^{\mathbf{G}} \Phi_{X_{1} \rightarrow X_{1}}^{\mathbf{E}_{1,2 j}[2]}\left(B_{1}\right)$ and $\Phi_{X_{1} \rightarrow X^{\prime}}^{\mathbf{G}} \Phi_{X_{1} \rightarrow X_{1}}^{\mathbf{E}_{1,2 j}[2]}\left(A_{1}\right)$ are locally free sheaves, and

$$
\begin{gathered}
\Phi_{X_{1} \rightarrow X^{\prime}}^{\mathbf{G}}\left(F_{2 j}^{\bullet}[1]\right)=\left[\Phi_{X_{1} \rightarrow X^{\prime}}^{\mathbf{G}} \Phi_{X_{1} \rightarrow X_{1}}^{\mathbf{E}_{1,2}[2]}\left(B_{1}\right) \rightarrow \Phi_{X_{1} \rightarrow X^{\prime}}^{\mathbf{G}} \Phi_{X_{1} \rightarrow X_{1}}^{\mathbf{E}_{1,2 j}[2]}\left(A_{1}\right)\right], \\
H^{i}\left(\Phi_{X_{1} \rightarrow X^{\prime}}^{\mathbf{G}}\left(F_{2 j}^{\bullet}[1]\right)\right)= \begin{cases}\Phi_{X_{1} \rightarrow X^{\prime}}^{\mathbf{G}} \Phi_{X_{0} \rightarrow X_{1}}^{\mathbf{E}_{0,2 j+1}}\left(A_{0}\right) & i=-1, \\
\Phi_{X_{1} \rightarrow X^{\prime}}^{\mathbf{G}} \Phi_{X_{0} \rightarrow X_{1}}^{\mathbf{E}_{0,2 j+1}[2]}\left(B_{0}\right) & i=0, \\
0, & i \neq-1,0 .\end{cases}
\end{gathered}
$$

- $\Phi_{X_{1} \rightarrow X^{\prime}}^{\mathbf{G}} \Phi_{X_{0} \rightarrow X_{1}}^{\mathbf{E}_{0,2 j+1}}\left(A_{0}^{\vee}[2]\right)$ and $\Phi_{X_{1} \rightarrow X^{\prime}}^{\mathbf{G}} \Phi_{X_{0} \rightarrow X_{1}}^{\mathbf{E}_{0,2 j+1}[2]}\left(B_{0}^{\vee}\right)$ are locally free sheaves, and

$$
\begin{aligned}
& \Phi_{X_{1} \rightarrow X^{\prime}}^{\mathrm{G}}\left(F_{2 j+1}^{\bullet}[1]\right)=\left[\Phi_{X_{1} \rightarrow X^{\prime}}^{\mathbf{G}} \Phi_{X_{0} \rightarrow X_{1}}^{\mathbf{E}_{0,2 j+1}}\left(A_{0}^{\vee}[2]\right) \rightarrow \Phi_{X_{1} \rightarrow X^{\prime}}^{\mathbf{G}} \Phi_{X_{0} \rightarrow X_{1}}^{\mathbf{E}_{0,2 j+1}[2]}\left(B_{0}^{\vee}\right)\right], \\
& H^{i}\left(\Phi_{X_{1} \rightarrow X^{\prime}}^{\mathbf{G}}\left(F_{2 j+1}^{\bullet}[1]\right)\right)= \begin{cases}\Phi_{X_{1} \rightarrow X^{\prime}}^{\mathbf{G}} \Phi_{X_{1} \rightarrow X_{1}}^{\mathbf{E}_{1,2 j+2}[2]}\left(B_{1}^{\vee}\right) & i=-1, \\
\Phi_{X_{1} \rightarrow X^{\prime}}^{\mathrm{G}} \Phi_{X_{1} \rightarrow X_{1}}^{\mathbf{E}_{1,2 j+2}[2]}\left(A_{1}^{\vee}[2]\right) & i=0, \\
0 & i \neq-1,0 .\end{cases}
\end{aligned}
$$

In particular we have exact sequences

$$
\begin{aligned}
\left(\mathfrak{\natural}_{2 j}\right) \quad 0 \rightarrow \Phi_{X_{1} \rightarrow X^{\prime}}^{\mathbf{G}} \Phi_{X_{0} \rightarrow X_{1}}^{\mathbf{E}_{0,2 j+1}}\left(A_{0}\right) \rightarrow \Phi_{X_{1} \rightarrow X^{\prime}}^{\mathbf{G}} \Phi_{X_{1} \rightarrow X_{1}}^{\mathbf{E}_{1,2 j}[2]}\left(B_{1}\right) & \\
& \rightarrow \Phi_{X_{1} \rightarrow X^{\prime}}^{\mathbf{G}} \Phi_{X_{1} \rightarrow X_{1}}^{\mathbf{E}_{1,2 j}[2]}\left(A_{1}\right) \rightarrow \Phi_{X_{1} \rightarrow X^{\prime}}^{\mathbf{G}} \Phi_{X_{0} \rightarrow X_{1}}^{\mathbf{E}_{0,2 j+1}[2]}\left(B_{0}\right) \rightarrow 0,
\end{aligned}
$$




$$
\begin{aligned}
\left(\natural_{2 j+1}\right) \quad 0 \rightarrow \Phi_{X_{1} \rightarrow X^{\prime}}^{\mathbf{G}} \Phi_{X_{1} \rightarrow X_{1}}^{\mathbf{E}_{1,2 j+2}[2]}\left(B_{1}^{\vee}\right) & \rightarrow \Phi_{X_{1} \rightarrow X^{\prime}}^{\mathbf{G}} \Phi_{X_{0} \rightarrow X_{1}}^{\mathbf{E}_{0,2 j+1}}\left(A_{0}^{\vee}[2]\right) \\
& \rightarrow \Phi_{X_{1} \rightarrow X^{\prime}}^{\mathbf{G}} \Phi_{X_{0} \rightarrow X_{1}}^{\mathbf{E}_{0,2 j+1}[2]}\left(B_{0}^{\vee}\right) \rightarrow \Phi_{X_{1} \rightarrow X^{\prime}}^{\mathbf{G}^{\prime}} \Phi_{X_{1} \rightarrow X_{1}}^{\mathbf{E}_{1,2 j+2}[2]}\left(A_{1}^{\vee}[2]\right) \rightarrow 0 .
\end{aligned}
$$

We note that $\chi\left(H^{1}\left(\Phi_{X_{1} \rightarrow X^{\prime}}^{\mathbf{G}}\left(F_{m}^{\bullet}\right)\right)\left(n H^{\prime}\right)\right), n \gg 0$ are decreasing for $0 \leq m \leq 2 k-1$. Finally for $\left.m=2 k, H^{1}\left(\Phi_{X_{1} \rightarrow X^{\prime}}^{\mathrm{G}}\left(F_{m}^{\bullet}\right)\right)\left(n H^{\prime}\right)\right)$ becomes zero. Thus $\Phi_{X_{1} \rightarrow X^{\prime}}^{\mathrm{G}}\left(F_{2 k}^{\bullet}\right)$ is a coherent sheaf and we have an exact sequence

$$
\begin{aligned}
0 \rightarrow \Phi_{X_{1} \rightarrow X^{\prime}}^{\mathbf{G}} \Phi_{X_{0} \rightarrow X_{1}}^{\mathbf{E}_{0,2 k+1}}\left(B_{0}\right) \rightarrow \Phi_{X_{1}}^{\mathbf{G}} & \rightarrow X^{\prime} \\
& \rightarrow \Phi_{X_{0} \rightarrow X_{1}}^{\mathbf{E}_{0,2 k+1}}\left(A_{0}\right) \\
& \rightarrow \Phi_{X_{1} \rightarrow X^{\prime}}^{\mathbf{G}} \Phi_{X_{1} \rightarrow X_{1}}^{\mathbf{E}_{1,2 k}[2]}\left(B_{1}\right) \rightarrow \Phi_{X_{1} \rightarrow X^{\prime}}^{\mathbf{G}} \Phi_{X_{1} \rightarrow X_{1}}^{\mathbf{E}_{1,2 k}[2]}\left(A_{1}\right) \rightarrow 0
\end{aligned}
$$

Hence the complex $\Phi_{X_{1} \rightarrow X^{\prime}}^{\mathrm{G}}\left(F_{m}^{\bullet}\right)$ eventually becomes a coherent sheaf as $m$ increases. By $\left(\natural_{m}\right)$, $\left(\natural_{m+1}\right)$ and (7.11), we see that the operation $\Phi_{X_{1} \rightarrow X^{\prime}}^{\mathrm{G}}\left(F_{m}^{\bullet}\right) \mapsto \Phi_{X_{1} \rightarrow X^{\prime}}^{\mathrm{G}}\left(F_{m+1}^{\bullet}\right)$ is nothing but the operation $\mathcal{F}$ in Fact 2.17. For example, if $m=2 j$, then by $\left(\Phi_{X_{1} \rightarrow X^{\prime}}^{\mathbf{G}} \Phi_{X_{1} \rightarrow X_{1}}^{\mathbf{E}_{0,2 j}[2]}\right)^{-1},\left(\mathfrak{\natural}_{2 j}\right)$ transforms to (\#). Then applying $\Phi_{X_{1} \rightarrow X^{\prime}}^{\mathbf{G}} \Phi_{X_{1} \rightarrow X_{1}}^{\mathbf{E}_{0,2 j}[2]} \mathcal{D}_{X_{1}}$, we get $\left(\natural_{2 j+1}\right)$.

7.4. Construction and the application of the tame complex: case II. Recall the notation in $\S \S 7.1$. Assume $\epsilon=q^{2}-\ell p^{2}=-1$. We will prove Proposition 7.11 for this case.

In the present case, the algebraic integers $a_{m}, b_{m}$ in Definition 7.9 satisfy

$$
0<\frac{b_{m}}{a_{m}}-\sqrt{\ell}<\frac{b_{m-1}}{a_{m-1}}-\sqrt{\ell}
$$

for $m \in \mathbb{Z}_{>0}$. We also have the following sequence of inequalities.

$$
\begin{aligned}
-\infty=\frac{b_{0}}{a_{0}}<-\frac{q}{p}=\frac{b_{-1}}{a_{-1}}<\frac{b_{-2}}{a_{-2}}<\cdots<-\sqrt{\ell}<\cdots<\frac{\ell a_{-2}}{b_{-2}}<\frac{\ell a_{-1}}{b_{-1}}=-\frac{\ell p}{q} \\
<\frac{\ell a_{0}}{b_{1}}=0<\frac{\ell a_{1}}{b_{1}}=\frac{\ell p}{q}<\frac{\ell a_{2}}{b_{2}}<\cdots<\sqrt{\ell}<\cdots<\frac{b_{2}}{a_{2}}<\frac{b_{1}}{a_{1}}=\frac{q}{p}<\frac{b_{0}}{a_{0}}=\infty .
\end{aligned}
$$

In this case we have a FMT $\Phi_{X_{0} \rightarrow X_{1}}^{\mathbf{E}}$ such that

$$
\theta\left(\Phi_{X_{0} \rightarrow X_{1}}^{\mathbf{E}}\right)=\left[\begin{array}{cc}
q & \ell p \\
p & q
\end{array}\right]
$$

Then Lemma 6.18 yields

$$
\theta\left(\Phi_{X_{1} \rightarrow X_{0}}^{\mathbf{E}}\right)=\left[\begin{array}{cc}
q & \ell p \\
p & q
\end{array}\right], \quad \theta\left(\Phi_{X_{1} \rightarrow X_{0}}^{\mathbf{E}^{\vee}[2]}\right)=\theta\left(\Phi_{X_{0} \rightarrow X_{1}}^{\mathbf{E}^{\vee}[2]}\right)=\left[\begin{array}{cc}
q & -\ell p \\
-p & q
\end{array}\right] .
$$

Definition 7.23. We define $\mathbf{E}_{i, m} \in \mathbf{D}\left(X_{i} \times X_{i+m}\right)(m \geq 0)$ inductively by

$$
\mathbf{E}_{i, 0}=\mathcal{O}_{\Delta}, \quad \Phi_{X_{i+m-1} \rightarrow X_{i+m}}^{\mathbf{E}^{\vee}[2]} \Phi_{X_{i} \rightarrow X_{i+m-1}}^{\mathbf{E}_{i, m-1}^{\vee}}=\Phi_{X_{i} \rightarrow X_{i+m}}^{\mathbf{E}_{i, m}^{\vee}} .
$$

Lemma 7.24. $\mathbf{E}_{i, m}$ is a locally free sheaf on $X_{i} \times X_{i+m}$ for $m>0$.

Proof. Assume that $\mathbf{E}_{i, m-1}$ is a locally free sheaf. We note that $\mu\left(\left.\mathbf{E}\right|_{X_{0} \times\left\{x_{1}\right\}} ^{\vee}\right)=\mu\left(\left.\mathbf{E}\right|_{\left\{x_{0}\right\} \times X_{1}} ^{\vee}\right)=$ $-\frac{q}{p} \sqrt{n}$. Since $\mu\left(\Phi_{X_{i} \rightarrow X_{i+m-1}}^{\mathbf{E}_{i, m-1}^{\vee}}\left(\mathbb{C}_{x_{i}}\right)\right)=-\frac{b_{m}}{a_{m}} \sqrt{n}$, we find that $\Phi_{X_{i+m-1} \rightarrow X_{i+m}}^{\mathbf{E}_{i, m-1}^{\vee}[2]} \Phi_{X_{i} \rightarrow X_{i+m-1}}^{\mathbf{E}^{\vee}}\left(\mathbb{C}_{x_{i}}\right)$ is a sheaf. Hence $\mathbf{E}_{i, m}$ is also a locally free sheaf.

Lemma 7.25. (1) For $m \in \mathbb{Z}_{>0}$, we have the following.

$$
\begin{aligned}
& \Phi_{X_{i} \rightarrow X_{i+m}}^{\mathbf{E}_{i,}^{\vee}[2]}=\Phi_{X_{i+m-1} \rightarrow X_{i+m}}^{\mathbf{E}^{\vee}[2]} \Phi_{X_{i+m-2} \rightarrow X_{i+m-1}}^{\mathbf{E}^{\vee}[2]} \cdots \Phi_{X_{i} \rightarrow X_{i+1}}^{\mathbf{E}^{\vee}[2]}, \\
& \Phi_{X_{i} \rightarrow X_{i+m}}^{\mathbf{E}_{i+m}}=\Phi_{X_{i+m-1} \rightarrow X_{i+m}}^{\mathbf{E}} \Phi_{X_{i+m-2} \rightarrow X_{i+m-1}}^{\mathbf{E}} \cdots \Phi_{X_{i} \rightarrow X_{i+1}}^{\mathbf{E}}, \\
& \Phi_{X_{i} \rightarrow X_{i+m}}^{\mathbf{E}_{i, m}}=\Phi_{X_{i+m-1} \rightarrow X_{i+m}}^{\mathbf{E}} \Phi_{X_{i+m-2} \rightarrow X_{i+m-1}}^{\mathbf{E}} \cdots \Phi_{X_{i} \rightarrow X_{i+1}}^{\mathbf{E}} .
\end{aligned}
$$


Under the identification $\xi: X_{i} \times X_{i+m} \rightarrow X_{i+m} \times X_{i}$, we also have

$$
\mathbf{E}_{i+m, m}=\mathbf{E}_{i, m} .
$$

(2) For $m \in \mathbb{Z}_{>0}$, we have the following.

$$
\theta\left(\Phi_{X_{i} \rightarrow X_{i+m}}^{\mathbf{E}_{i, m}^{\vee}[2]}\right)=\left[\begin{array}{cc}
b_{m} & -\ell a_{m} \\
-a_{m} & b_{m}
\end{array}\right]=\left[\begin{array}{cc}
b_{-m} & \ell a_{-m} \\
a_{-m} & b_{-m}
\end{array}\right], \quad \theta\left(\Phi_{X_{i} \rightarrow X_{i+m}}^{\mathbf{E}_{i, m}}\right)=\left[\begin{array}{cc}
b_{m} & \ell a_{m} \\
a_{m} & b_{m}
\end{array}\right] .
$$

Proof. (1) The first claim is obvious from Definition 7.23. For the second and third one, the computation proceed as follows.

$$
\begin{aligned}
\Phi_{X_{i} \rightarrow X_{i+m}}^{\mathbf{E}_{i+m, m}} & =\left(\Phi_{X_{i+m} \rightarrow X_{i+2 m}}^{\mathbf{E}_{i+m, m}^{\vee}[2]}\right)^{-1} \\
& =\left(\Phi_{X_{i+2 m-1} \rightarrow X_{i+2 m}}^{\mathbf{E}^{\vee}[2]} \Phi_{X_{i+2 m-2} \rightarrow X_{i+2 m-1}}^{\mathbf{E}^{\vee}[2]} \cdots \Phi_{X_{i+m} \rightarrow X_{i+m+1}}^{\mathbf{E}^{\vee}[2]}\right)^{-1} \\
& =\Phi_{X_{i+m-1} \rightarrow X_{i+m}}^{\mathbf{E}} \Phi_{X_{i+m-2} \rightarrow X_{i+m-1}}^{\mathbf{E}} \cdots \Phi_{X_{i} \rightarrow X_{i+1}}^{\mathbf{E}}, \\
\Phi_{X_{i} \rightarrow X_{i+m}}^{\mathbf{E}_{i, m}} & =\mathcal{D}_{X_{i+m}} \Phi_{X_{i, m} \rightarrow X_{i+m}}^{\mathbf{E}^{\vee}[2]} \mathcal{D}_{X_{i}} \\
& =\mathcal{D}_{X_{i+m}} \Phi_{X_{i+m-1} \rightarrow X_{i+m}}^{\mathbf{E}^{\vee}[2]} \Phi_{X_{i+m-2} \rightarrow X_{i+m-1}}^{\mathbf{E}^{\vee}[2]} \cdots \Phi_{X_{i} \rightarrow X_{i+1}}^{\mathbf{E}^{\vee}[2]} \mathcal{D}_{X_{i}} \\
& =\Phi_{X_{i+m-1} \rightarrow X_{i+m}}^{\mathbf{E}} \Phi_{X_{i+m-2} \rightarrow X_{i+m-1}}^{\mathbf{E}} \cdots \Phi_{X_{i} \rightarrow X_{i+1}}^{\mathbf{E}} .
\end{aligned}
$$

The last claim is a consequence of the rest ones.

(2) This is a consequence of (1), (7.13) and (7.14).

In the present case $\epsilon=q^{2}-\ell p^{2}=1, F \in M_{X}^{H}(1,0,-\ell)$ has the cokernel presentation

$$
0 \rightarrow \Phi_{X_{0} \rightarrow X_{1}}^{\mathbf{E}}\left(A_{0}\right)^{\vee} \rightarrow \Phi_{X_{0} \rightarrow X_{1}}^{\mathbf{E}}\left(B_{0}\right)^{\vee} \rightarrow F \rightarrow 0 .
$$

Thus we have an exact sequence

$$
0 \rightarrow \Phi_{X_{0} \rightarrow X_{1}}^{\mathbf{E}}\left(A_{0}\right)^{\vee} \rightarrow \Phi_{X_{0} \rightarrow X_{1}}^{\mathbf{E}}\left(B_{0}\right)^{\vee} \rightarrow B_{1} \rightarrow A_{1} \rightarrow 0 .
$$

Applying $\Phi_{X_{1} \rightarrow X_{0}}^{\mathbf{E}^{\vee}}$ to the dual of (D) , we get

$$
0 \rightarrow \Phi_{X_{1} \rightarrow X_{0}}^{\mathbf{E}^{\vee}[2]}\left(A_{1}^{\vee}\right) \rightarrow \Phi_{X_{1} \rightarrow X_{0}}^{\mathbf{E}^{\vee}[2]}\left(B_{1}^{\vee}\right) \rightarrow B_{0} \rightarrow A_{0} \rightarrow 0 .
$$

Now we can define the tame complex for the case $\epsilon=1$.

Definition 7.26. For $F:=\operatorname{Ker}\left(B_{1} \rightarrow A_{1}\right)$, we set the tame complex $F_{m}^{\bullet}$ by

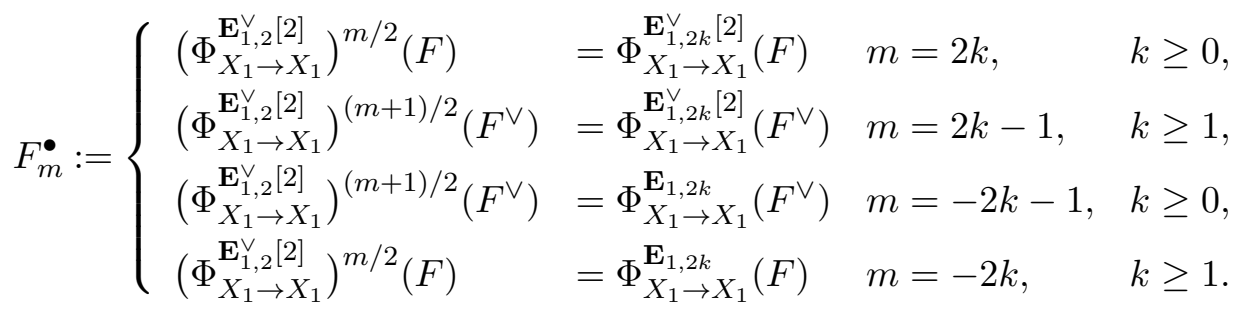

We can rewrite $F_{m}^{\bullet}$ as follows.

\section{Lemma 7.27.}

$$
F_{m}^{\bullet}= \begin{cases}\Phi_{X_{0,2 k+1} \rightarrow X_{1}}^{\mathbf{E}_{0}^{\vee}}\left(\left[A_{0}^{\vee} \rightarrow B_{0}^{\vee}\right]\right) & m=2 k, \\ \Phi_{X_{0} \rightarrow X_{1}}^{\mathbf{E}_{0}}\left(\left[A_{0}^{\vee} \rightarrow B_{0}^{\vee}\right]^{\vee}\right) & m=2 k-1, \\ \Phi_{X_{0} \rightarrow X_{1}}^{\mathbf{E}_{1,2 k+1}}\left(\left[A_{0}^{\vee} \rightarrow B_{0}^{\vee}\right]^{\vee}\right) & m=-2 k-1, \\ \Phi_{X_{0} \rightarrow X_{1}}^{\mathbf{E}_{1,2 k-1}}\left(\left[A_{0}^{\vee} \rightarrow B_{0}^{\vee}\right]\right) & m=-2 k .\end{cases}
$$

Proof. This is the consequence of the definition and Lemma 7.28 below. 


\section{Lemma 7.28.}

$$
\begin{array}{ll}
\Phi_{X_{1} \rightarrow X_{1+m}}^{\mathbf{E}_{1, m}^{\vee}[2]} \mathcal{D}_{X_{1}} \Phi_{X_{0} \rightarrow X_{1}}^{\mathbf{E}} & =\Phi_{X_{0} \rightarrow X_{m+1}}^{\mathbf{E}_{0, m+1}^{\vee}[2]} \mathcal{D}_{X_{0}}, \\
\left(\Phi_{X_{1} \rightarrow X_{1+m}}^{\mathbf{E}_{1}[2]} \mathcal{D}_{X_{1}}\right)\left(\mathcal{D}_{X_{1}} \Phi_{X_{0} \rightarrow X_{1}}^{\mathbf{E}}\right) & =\Phi_{X_{0} \rightarrow X_{m-1}}^{\mathbf{E}_{0}[2]}, \\
\Phi_{X_{1} \rightarrow X_{1+m}}^{\mathbf{E}_{1, m}} \mathcal{D}_{X_{1}} \Phi_{X_{0} \rightarrow X_{1}}^{\mathbf{E}} & =\Phi_{X_{0} \rightarrow X_{m-1}}^{\mathbf{E}_{0, m}} \mathcal{D}_{X_{0}}, \\
\left(\Phi_{X_{1} \rightarrow X_{1+m}}^{\mathbf{E}_{1, m}} \mathcal{D}_{X_{1}}\right)\left(\mathcal{D}_{X_{1}} \Phi_{X_{0} \rightarrow X_{1}}^{\mathbf{E}}\right) & =\Phi_{X_{0} \rightarrow X_{1+m}}^{\mathbf{E}_{0,1+m}} .
\end{array}
$$

Proof. The second and the last equality follows from the definitions of $E_{i, m}$. For the remained equations, we have

$$
\begin{aligned}
& \Phi_{X_{1} \rightarrow X_{1+m}}^{\mathbf{E}_{1, m}^{\vee}[2]} \mathcal{D}_{X_{1}} \Phi_{X_{0} \rightarrow X_{1}}^{\mathbf{E}}=\Phi_{X_{1} \rightarrow X_{1+m}}^{\mathbf{E}_{1, m}^{\vee}[2]} \Phi_{X_{0} \rightarrow X_{1}}^{\mathbf{E}^{\vee}[2]} \mathcal{D}_{X_{0}}=\Phi_{X_{0} \rightarrow X_{m+1}}^{\mathbf{E}_{0, m+1}^{\vee}[2]} \mathcal{D}_{X_{0}}, \\
& \Phi_{X_{1} \rightarrow X_{1+m}}^{\mathbf{E}_{1, m}} \mathcal{D}_{X_{1}} \Phi_{X_{0} \rightarrow X_{1}}^{\mathbf{E}}=\Phi_{X_{1} \rightarrow X_{1+m}}^{\mathbf{E}_{1, m}} \Phi_{X_{0} \rightarrow X_{1}}^{\mathbf{E}^{\vee}[2]} \mathcal{D}_{X_{0}}=\Phi_{X_{0} \rightarrow X_{m-1}}^{\mathbf{E}_{0, m-1}} \mathcal{D}_{X_{0}} .
\end{aligned}
$$

For the proof of Proposition 7.11, we prepare some calculations.

\section{Lemma 7.29.}

$$
\Phi_{X_{i} \rightarrow X_{i+m}}^{\mathbf{E}_{i, m}^{\vee}[2]}\left(B_{i}\right), \Phi_{X_{i} \rightarrow X_{i+m}}^{\mathbf{E}_{i, m}^{\vee}[2]}\left(B_{i}^{\vee}\right), \Phi_{X_{i} \rightarrow X_{i+m}}^{\mathbf{E}_{i, m}}\left(B_{i}\right), \Phi_{X_{i} \rightarrow X_{i+m}}^{\mathbf{E}_{i, m}}\left(B_{i}^{\vee}\right) \in \operatorname{Coh}\left(X_{i+m}\right) .
$$

Proof. Since $\mu\left(\left.\mathbf{E}_{i, m}\right|_{\left\{x_{i}\right\} \times X_{i+m}}\right)=\mu\left(\left.\mathbf{E}_{i, m}\right|_{X_{i} \times\left\{x_{i+m}\right\}}\right)=b_{m} / a_{m}>0$ for $m \geq 1$ and $\operatorname{deg}\left(B_{i}\right)=0$, we get our claim.

Proof of Proposition 7.11. By Lemma 7.29, we have the following exact sequences for $k \geq 0$.

$$
0 \rightarrow \Phi_{X_{1} \rightarrow X_{1}}^{\mathbf{E}_{1,2 k+2}^{\vee}}\left(A_{1}\right) \rightarrow \Phi_{X_{0} \rightarrow X_{1}}^{\mathbf{E}_{0,2 k+3}^{\vee}}\left(A_{0}^{\vee}[2]\right) \rightarrow \Phi_{X_{0} \rightarrow X_{1}}^{\mathbf{E}_{0,2 k+3}^{\vee}[2]}\left(B_{0}^{\vee}\right) \rightarrow \Phi_{X_{1} \rightarrow X_{1}}^{\mathbf{E}_{1,2 k+2}^{\vee}[2]}\left(B_{1}\right) \rightarrow 0,
$$

$\left(b_{2 k+1}\right)$

$$
0 \rightarrow \Phi_{X_{0} \rightarrow X_{1}}^{\mathbf{E}_{0,2 k+1}^{\vee}}\left(A_{0}\right) \rightarrow \Phi_{X_{1} \rightarrow X_{1}}^{\mathbf{E}_{1,2 k+2}}\left(A_{1}^{\vee}[2]\right) \rightarrow \Phi_{X_{1} \rightarrow X_{1}}^{\mathbf{E}_{1,2 k+2}^{\vee}[2]}\left(B_{1}^{\vee}\right) \rightarrow \Phi_{X_{0} \rightarrow X_{1}}^{\mathbf{E}_{0,2 k+1}^{\vee}[2]}\left(B_{0}\right) \rightarrow 0,
$$

$\left(b_{-2 k-1}\right)$

$$
0 \rightarrow \Phi_{X_{1} \rightarrow X_{1}}^{\mathbf{E}_{1,2 k}}\left(B_{1}^{\vee}\right) \rightarrow \Phi_{X_{0} \rightarrow X_{1}}^{\mathbf{E}_{0,2 k+1}}\left(B_{0}\right) \rightarrow \Phi_{X_{0} \rightarrow X_{1}}^{\mathbf{E}_{0,2 k+1}}\left(A_{0}\right) \rightarrow \Phi_{X_{1} \rightarrow X_{1}}^{\mathbf{E}_{1,2 k}}\left(A_{1}^{\vee}[2]\right) \rightarrow 0,
$$

$(b-2 k-2)$

$$
0 \rightarrow \Phi_{X_{0} \rightarrow X_{1}}^{\mathbf{E}_{0,2 k+1}}\left(B_{0}^{\vee}\right) \rightarrow \Phi_{X_{1} \rightarrow X_{1}}^{\mathbf{E}_{1,2 k+2}}\left(B_{1}\right) \rightarrow \Phi_{X_{1} \rightarrow X_{1}}^{\mathbf{E}_{1,2 k+2}}\left(A_{1}\right) \rightarrow \Phi_{X_{0} \rightarrow X_{1}}^{\mathbf{E}_{0,2 k+1}}\left(A_{0}^{\vee}[2]\right) \rightarrow 0 .
$$

These exact sequences and Lemma 7.27 give the following relations of FMTs.

$$
\begin{aligned}
& F_{2 k}^{\bullet}=\Phi_{X_{1,2 k} \rightarrow X_{1}}^{\mathbf{E}_{\vee}^{\vee}} \mathcal{D}_{X_{1}}\left(\Phi_{X_{1} \rightarrow X_{1}}^{\mathbf{E}_{1,2 k}^{\vee}}\right)^{-1}\left(F_{2 k-1}^{\bullet}\right), \\
& F_{2 k+1}^{\bullet}=\Phi_{X_{0} \rightarrow X_{1}}^{\bullet} \mathcal{D}_{X_{0}}\left(\Phi_{X_{0} \rightarrow X_{1}}^{\mathbf{E}_{0}}\right)^{-1}\left(F_{-2 k-1}^{\bullet}\right), \\
& F_{-2 k-1}^{\bullet}=\Phi_{X_{1} \rightarrow X_{1}}^{\mathbf{E}_{1,2 k}} \mathcal{D}_{X_{1}}\left(\Phi_{X_{1} \rightarrow X_{1}}^{\mathbf{E}_{1,2 k}}\right)^{-1}\left(F_{-2 k}^{\bullet}\right), \\
& F_{-2 k-2}^{\bullet}=\Phi_{X_{0} \rightarrow X_{1}}^{\mathbf{E}_{0,2 k+1}} \mathcal{D}_{X_{0}}\left(\Phi_{X_{0} \rightarrow X_{1}}^{\mathbf{E}_{0,2 k+1}}\right)^{-1}\left(F_{-2 k-1}^{\bullet}\right.
\end{aligned}
$$

From these relation, we can easily get the claim.

Definition 7.30. We set

$$
\begin{array}{lll}
I_{0}:=\left(0, \frac{\ell a_{1}}{b_{1}}\right] \cup\left(\frac{b_{1}}{a_{1}}, \infty\right], & I_{-1} & :=\left(-\infty,-\frac{b_{1}}{a_{1}}\right] \cup\left(-\frac{\ell a_{1}}{b_{1}}, 0\right], \\
I_{m}:=\left(\frac{\ell a_{m}}{b_{m}}, \frac{\ell a_{m+1}}{b_{m+1}}\right] \cup\left(\frac{b_{m+1}}{a_{m+1}}, \frac{b_{m}}{a_{m}}\right], & I_{-m-1}:=\left(-\frac{b_{m}}{a_{m}},-\frac{b_{m+1}}{a_{m+1}}\right] \cup\left(-\frac{\ell a_{m+1}}{b_{m+1}},-\frac{\ell a_{m}}{b_{m}}\right] \quad m \geq 1 .
\end{array}
$$

Then we have $\mathbb{P}^{1}(\mathbb{R}) \backslash\{ \pm \sqrt{\ell}\}=\coprod_{m \in \mathbb{Z}} I_{m}=\coprod_{m \in \mathbb{Z}} I_{m}^{*}$.

Theorem 7.31. For a FMT $\Phi_{X_{1} \rightarrow X^{\prime}}^{\mathbf{G}}: \mathbf{D}\left(X_{1}\right) \rightarrow \mathbf{D}\left(X^{\prime}\right)$, we set $\lambda:=\mu\left(\mathbf{G}_{x^{\prime}}\right) / \sqrt{n}$.

(1) If $\lambda \in I_{m}$, then $\Phi_{X_{1} \rightarrow X^{\prime}}^{\mathbf{G}}\left(F_{m}^{\bullet}\right)$ is a coherent sheaf.

(2) If $\lambda \in I_{m}^{*}$, then $\mathcal{D}_{X^{\prime}} \Phi_{X_{1} \rightarrow X^{\prime}}^{\mathrm{G}}\left(F_{m}^{\bullet}\right)=\Phi_{X_{1} \rightarrow X^{\prime}}^{\mathbf{G}^{\vee}[2]} \mathcal{D}_{X_{1}}\left(F_{m}^{\bullet}\right)$ is a coherent sheaf. 
The proof is based on the calculation in this subsection and is similar to that of Theorem 7.21. We omit the detail.

Remark 7.32. In [M2, Theorem 3] Mukai proved the case $\ell=2, n=1$ of our Theorem 7.21, He also claims that the operation $\mathcal{F}$ in Remark 7.22 gives an isomorphism between the moduli and the Hilbert scheme. We will return to this point in our future work.

\section{Birational Morphism of The MOduli}

In this section we give an explicit description of the birational map between the moduli of stable sheaves and the Hilbert scheme of points: $M_{X}^{H}(v) \cdots \rightarrow X \times \mathrm{Hilb}^{\left\langle v^{2}\right\rangle / 2}(X)$. Fix an abelian surface $X$ with $\operatorname{NS}(X)=\mathbb{Z} H$ and set $n:=\left(H^{2}\right) / 2$.

8.1. Quadratic forms and the description of the birational map. Recall the matrix description introduced in $\S \S 6.3$. We begin with the next simple remark.

Lemma 8.1. For an isotropic Mukai vector $w=\left(p^{2}, \frac{p q}{\sqrt{n}} H, q^{2}\right)$ and a Mukai vector $v=(r, d H, a)$,

$$
\langle w, v\rangle=Q_{v}(q,-p) .
$$

Here the symbol $Q_{v}$ is the quadratic form defined to be

$$
Q_{v}(x, y):=-\left(r x^{2}+2 d \sqrt{n} x y+a y^{2}\right)=-\left[\begin{array}{ll}
x & y
\end{array}\right]\left[\begin{array}{cc}
r & d \sqrt{n} \\
d \sqrt{n} & a
\end{array}\right]\left[\begin{array}{l}
x \\
y
\end{array}\right] .
$$

Proof.

$$
\begin{aligned}
\langle w, v\rangle & =B\left(\left[\begin{array}{cc}
p^{2} & p q \\
p q & q^{2}
\end{array}\right],\left[\begin{array}{cc}
r & d \sqrt{n} \\
d \sqrt{n} & a
\end{array}\right]\right)=-\operatorname{tr}\left(\left[\begin{array}{cc}
q^{2} & -p q \\
-p q & p^{2}
\end{array}\right]\left[\begin{array}{cc}
r & d \sqrt{n} \\
d \sqrt{n} & a
\end{array}\right]\right) \\
& =-\operatorname{tr}\left(\left[\begin{array}{c}
q \\
-p
\end{array}\right]\left[\begin{array}{ll}
q & -p
\end{array}\right]\left[\begin{array}{cc}
r & d \sqrt{n} \\
d \sqrt{n} & a
\end{array}\right]\right)=-\operatorname{tr}\left(\left[\begin{array}{cc}
q & -p
\end{array}\right]\left[\begin{array}{cc}
r & d \sqrt{n} \\
d \sqrt{n} & a
\end{array}\right]\left[\begin{array}{c}
q \\
-p
\end{array}\right]\right) \\
& =Q_{v}(q,-p) .
\end{aligned}
$$

Proposition 8.2. Fix a positive Mukai vector $v=(r, d H, a)$ and assume $\ell:=\left\langle v^{2}\right\rangle / 2$ is positive. Assume further that there exists a solution $\left(p_{1}, q_{1}\right)$ of the quadratic indefinite equation

$$
Q_{v}\left(q_{1},-p_{1}\right)=-a p_{1}^{2}+2 d \sqrt{n} p_{1} q_{1}-r q_{1}^{2}=\epsilon, \quad \epsilon= \pm 1,
$$

with the additional condition

$$
p_{1}^{2}, p_{1} q_{1} / \sqrt{n}, q_{1}^{2} \in \mathbb{Z}
$$

We also set

$$
p_{2}:=\epsilon\left(d \sqrt{n} p_{1}-r q_{1}\right), \quad q_{2}:=\epsilon\left(-d \sqrt{n} q_{1}+a p_{1}\right) .
$$

(1) Take $\left(p_{1}, q_{1}\right)$ satisfying (8.2) such that $\left|p_{1}\right|$ is minimum among them. Then a general member of $M_{X}^{H}(v)$ has the next semi-homogeneous presentation.
(a) $\operatorname{Ker}\left(E_{1} \rightarrow E_{2}\right) \quad$ if $-p_{2} / p_{1} \geq 0$ and $\epsilon=+1$,
(b) $\operatorname{Ker}\left(E_{2} \rightarrow E_{1}\right) \quad$ if $-p_{2} / p_{1}<0$ and $\epsilon=-1$,
(c) $\operatorname{Coker}\left(E_{1} \rightarrow E_{2}\right)$ if $-p_{2} / p_{1} \geq 0$ and $\epsilon=-1$,
(d) $\operatorname{Coker}\left(E_{2} \rightarrow E_{1}\right)$ if $-p_{2} / p_{1}<0$ and $\epsilon=+1$.

Here $E_{i}$ are semi-homogeneous sheaves with $v\left(E_{1}\right)=\ell\left(p_{1}^{2}, \frac{p_{1} q_{1}}{\sqrt{n}} H, q_{1}^{2}\right)$ and $v\left(E_{2}\right)=$ $\left(p_{2}^{2}, \frac{p_{2} q_{2}}{\sqrt{n}} H, q_{2}^{2}\right)$. 
(2) The element

$$
g:=\left[\begin{array}{cc}
q_{1} & -q_{2} \\
-p_{1} & p_{2}
\end{array}\right]
$$

belongs to $G$, and we can take a universal family $\mathbf{F}$ on $X \times X^{\prime}$ such that $\theta\left(\Phi_{X \rightarrow X^{\prime}}^{\mathbf{F}}\right)= \pm g \in$ $\operatorname{PSL}(2, \mathbb{R})$. Then the following functor induces the birational map $M_{X}^{H}(r, d H, a) \cdots \rightarrow$ $M_{X^{\prime}}^{H^{\prime}}(1,0,-\ell)$ for each case $(\mathrm{a})-(\mathrm{d})$.
(a) $\mathcal{D}_{X^{\prime}} \Phi_{X \rightarrow X^{\prime}}^{\mathbf{F}[1]}$,
(b) $\Phi_{X \rightarrow X^{\prime}}^{\mathbf{F}[-2]}$
(c) $\mathcal{D}_{X^{\prime}} \Phi_{X \rightarrow X^{\prime}}^{\mathbf{F}}$,
(d) $\Phi_{X \rightarrow X^{\prime}}^{\mathbf{F}[-1]}$

By this functor, the semi-homogeneous sheaves $E_{1}, E_{2}$ appearing in (1) are transformed in to $L \otimes \mathcal{O}_{Z}$ and $L$, where $Z$ is a 0 -dimensional subscheme of $X$ with length $\ell$ and $L$ is a line bundle on $X$.

Proof. The condition (8.1) and (8.2) immediately show $g \in G$, so that $\Phi_{X \rightarrow X^{\prime}}^{\mathbf{F}}$ does exist. Since the rest statements of (2) follow from those of (1), we give the proof of (1).

Lemma 8.1 shows that the equation (8.1) is equivalent to the numerical equation for $v$. By Theorem 5.2, we know that a general member of $M_{X}^{H}(v)$ has a semi-homogeneous presentation which associates to the numerical solution of minimal rank. Then Proposition 3.2 means that the statements follows from the WIT with respect to $\Phi_{X \rightarrow X^{\prime}}^{\mathrm{F}}$. We study the WIT with respect to the inverse transform $\Phi_{X^{\prime} \rightarrow X}^{\mathbf{F}^{\vee}}$, since it is more convenient.

A simple calculation shows that

$$
\begin{aligned}
& { }^{t} g^{-1}\left[\begin{array}{ll}
0 & 0 \\
0 & 1
\end{array}\right] g^{-1}=\left[\begin{array}{cc}
p_{1}^{2} & p_{1} q_{1} \\
p_{1} q_{1} & q_{1}^{2}
\end{array}\right], \quad{ }^{t} g^{-1}\left[\begin{array}{ll}
1 & 0 \\
0 & 0
\end{array}\right] g^{-1}=\left[\begin{array}{cc}
p_{2}^{2} & p_{2} q_{2} \\
p_{2} q_{2} & q_{2}^{2}
\end{array}\right], \\
& { }^{t} g^{-1}\left[\begin{array}{cc}
1 & 0 \\
0 & -\ell
\end{array}\right] g^{-1}=-\epsilon\left[\begin{array}{cc}
r & d \sqrt{n} \\
d \sqrt{n} & a
\end{array}\right] .
\end{aligned}
$$

Then Proposition 6.19 and the equality $\theta\left(\Phi_{X^{\prime} \rightarrow X}^{\mathbf{F}^{\vee}}\right)= \pm g^{-1}= \pm\left[\begin{array}{ll}p_{2} & q_{2} \\ p_{1} & q_{1}\end{array}\right]$ yield

$$
\begin{aligned}
& v\left(\Phi_{X^{\prime} \rightarrow X}^{\mathbf{F}^{\vee}}\left(\mathbb{C}_{X^{\prime}}\right)\right)=\left(p_{1}^{2}, \frac{p_{1} q_{1}}{\sqrt{n}} H, q_{1}^{2}\right), \quad v\left(\Phi_{X^{\prime} \rightarrow X}^{\mathbf{F}^{\vee}}\left(\mathcal{O}_{X^{\prime}}\right)\right)=\left(p_{2}^{2}, \frac{p_{2} q_{2}}{\sqrt{n}} H, q_{2}^{2}\right), \\
& v\left(\Phi_{X^{\prime} \rightarrow X}^{\mathbf{F}^{\vee}}\left(I_{Z} \otimes L\right)\right)=v\left(E_{2}\right)-v\left(E_{1}\right),
\end{aligned}
$$

where we used symbols $x^{\prime} \in X^{\prime}$ and $L \in \operatorname{Pic}^{0}\left(X^{\prime}\right)$ and the symbol $Z$ means a 0-dimensional subscheme of length $\ell$. We also find that WIT $\mathrm{W}_{0}$ holds for $\mathcal{O}_{X^{\prime}}$ with respect to $\Phi_{X^{\prime} \rightarrow X}^{\mathbf{F}^{\vee}}$ if and only if $-p_{2} / p_{1}<0$, since $\mu\left(\left.\mathbf{F}\right|_{\{x\} \times X^{\prime}}\right)=-p_{2} / p_{1}$.

Hence if $\mu\left(\left.\mathbf{F}\right|_{\{x\} \times X^{\prime}}\right)<0$, then both $\Phi_{X^{\prime} \rightarrow X}^{\mathbf{F}^{\vee}}\left(\mathbb{C}_{x^{\prime}}\right)$ and $\Phi_{X^{\prime} \rightarrow X}^{\mathbf{F}^{\vee}}\left(\mathcal{O}_{X^{\prime}}\right)$ are semi-homogeneous sheaves. By the minimality of $\left|p_{1}\right|, F^{\bullet}:=\Phi_{X^{\prime} \rightarrow X}^{\mathbf{F}^{\vee}}\left(I_{Z} \otimes L\right)$ is a coherent sheaf up to shift for a general $I_{Z} \otimes L$. The shift is determined by the sign of $\operatorname{rk}\left(F^{\bullet}\right)=\ell p_{1}^{2}-p_{2}^{2}$, which equals to $\epsilon r$ by the definition of $p_{2}$ and (8.1). Thus we have the statements for the cases (b) and (d).

If $\mu\left(\left.\mathbf{F}\right|_{\{x\} \times X^{\prime}}\right) \geq 0$, then both $\Phi_{X^{\prime} \rightarrow X}^{\mathbf{F}^{\vee}[2]} \mathcal{D}_{X^{\prime}}\left(\mathbb{C}_{x^{\prime}}\right)$ and $\Phi_{X^{\prime} \rightarrow X}^{\mathbf{F}^{\vee}[2]} \mathcal{D}_{X^{\prime}}\left(\mathcal{O}_{X^{\prime}}\right)$ are semi-homogeneous sheaves. Similar arguments yield the statements for (a) and (c).

8.2. Structures of $G$. As the preliminary of the next subsection, we study the structure of the arithmetic group $G$ introduced in Definition 6.9.

Definition 8.3. Let $n=p_{1}^{e_{1}} p_{2}^{e_{2}} \cdots p_{N}^{e_{N}}$ be the prime decomposition of the integer $n:=\left(H^{2}\right) / 2$. We define a map $\widetilde{\phi}_{i}: \mathbb{Z} \rightarrow\{0,1\}$ sending $m$ to

$$
\widetilde{\phi}_{i}(m)= \begin{cases}1 & p_{i} \mid m, \\ 0 & p_{i} \backslash \backslash m .\end{cases}
$$


We identify $\{0,1\}$ with $\mathbb{Z} / 2 \mathbb{Z}$ in a natural way, and regard it as a group. Then we also define a map:

$$
\begin{array}{rlc}
\widetilde{\phi}: & \mathbb{Z} & \rightarrow \\
m & \left.\mapsto\left(\widetilde{\phi}_{1}(m), \widetilde{\mathbb{\phi}}_{2}(m), \ldots \mathbb{Z}\right)^{\oplus N}, \widetilde{\phi}_{N}(m)\right) .
\end{array}
$$

Clearly this $\widetilde{\phi}$ is a group homomorphism. Then for $g=\left[\begin{array}{ll}a \sqrt{r} & b \sqrt{s} \\ c \sqrt{s} & d \sqrt{r}\end{array}\right] \in G$, we set $\phi(g):=\widetilde{\phi}(r)$. By Lemma 6.8, $r$ is uniquely determined by $g$. Thus $\phi(g)$ is well-defined.

Lemma 8.4. $\phi\left(g g^{\prime}\right)=\phi(g)+\phi\left(g^{\prime}\right)$. Thus $\phi: G \rightarrow(\mathbb{Z} / 2 \mathbb{Z})^{\oplus N}$ is a homomorphism.

Proof. We use the notation in (6.13). We set $r=p r_{1}, r^{\prime}=p r_{1}^{\prime}, \operatorname{gcd}\left(r_{1}, r_{1}^{\prime}\right)=1$ and $s=q s_{1}$, $s^{\prime}=q s_{1}^{\prime}, \operatorname{gcd}\left(s_{1}, s_{1}^{\prime}\right)=1$. Then $n=p q r_{1} s_{1}=p q r_{1}^{\prime} s_{1}^{\prime}$. Hence $r_{1}^{\prime}=s_{1}$ and $s_{1}^{\prime}=r_{1}$. Thus

$$
\left[\begin{array}{ll}
a \sqrt{r} & b \sqrt{s} \\
c \sqrt{s} & d \sqrt{r}
\end{array}\right]\left[\begin{array}{ll}
a^{\prime} \sqrt{r^{\prime}} & b^{\prime} \sqrt{s^{\prime}} \\
c^{\prime} \sqrt{s^{\prime}} & d^{\prime} \sqrt{r^{\prime}}
\end{array}\right]=\left[\begin{array}{ll}
\left(a a^{\prime} p+b c^{\prime} q\right) \sqrt{r_{1} s_{1}} & \left(a b^{\prime} r_{1}+b d^{\prime} s_{1}\right) \sqrt{p q} \\
\left(c a^{\prime} s_{1}+d c^{\prime} r_{1}\right) \sqrt{p q} & \left(c b^{\prime} q+d d^{\prime} p\right) \sqrt{r_{1} s_{1}}
\end{array}\right]
$$

By the uniqueness of the description (Lemma 6.8), $\phi\left(g g^{\prime}\right)=\widetilde{\phi}\left(r_{1} s_{1}\right)=\widetilde{\phi}\left(r_{1} r_{1}^{\prime}\right)=\widetilde{\phi}\left(p r_{1}\right)+$ $\widetilde{\phi}\left(p r_{1}^{\prime}\right)=\phi(g)+\phi\left(g^{\prime}\right)$.

Proposition 8.5. (1) $\phi$ is surjective and $\operatorname{ker} \phi$ is isomorphic to the modular group $\Gamma_{0}(n)$.

(2) For $g=\left[\begin{array}{ll}a \sqrt{r} & b \sqrt{s} \\ c \sqrt{s} & d \sqrt{r}\end{array}\right] \in G$ and $Y \in \operatorname{FM}(X)$, we set $Y_{g^{-1}}:=M_{Y}^{\widehat{H}}\left(c^{2} s, c d \widehat{H}, d^{2} r\right)$, where $\widehat{H}$ is the ample generator of $\mathrm{NS}(Y)$. Then $\left(Y_{g^{-1}}\right)_{g^{\prime-1}} \cong Y_{\left(g^{\prime} g\right)^{-1}}$ and $Y_{g^{-1}}$ is determined by $\phi(g)$.

(3) Assume that $\operatorname{rk} \operatorname{End}(X)=1$. Then $Y_{g^{-1}} \cong Y$ if and only if $g \in \operatorname{ker} \phi$. In particular, $\operatorname{ker} \phi=\theta\left(\mathrm{Eq}_{0}(\mathbf{D}(Y), \mathbf{D}(Y))\right)$.

Proof. (1) The surjectivity is easy. We note that

$$
\operatorname{ker} \phi=\left\{\left[\begin{array}{cc}
a & b \sqrt{n} \\
c \sqrt{n} & d
\end{array}\right] \mid a, b, c, d \in \mathbb{Z}, a d-b c n=1\right\} .
$$

It is a conjugate of $\Gamma_{0}(n)$. Thus the second claim also holds.

(2) We have a FMT $\Phi_{g}: \mathbf{D}\left(Y_{g^{-1}}\right) \rightarrow \mathbf{D}(Y)$ such that $\theta\left(\Phi_{g}\right)=g$. Since the composition $\Phi_{g} \Phi_{g^{\prime}}$ : $\mathbf{D}\left(\left(Y_{g^{-1}}\right)_{g^{\prime-1}}\right) \rightarrow \mathbf{D}\left(Y_{g^{-1}}\right) \rightarrow \mathbf{D}(Y)$ satisfies $\theta\left(\Phi_{g} \Phi_{g^{\prime}}\right)=g^{\prime} g=\theta\left(\Phi_{g^{\prime} g}\right)$, we have $\left(Y_{g^{-1}}\right)_{g^{\prime-1}} \cong$ $Y_{\left(g^{\prime} g\right)^{-1}}$. The second claim follows from the following Lemma 8.6.

(3) is due to Orlov [Or, Example 4.16]. See also the argument in the next Lemma 8.6.

Lemma 8.6. Let $p, q \in \mathbb{Z}$ and $r, s \in \mathbb{Z}_{>0}$ with $r s=n:=\left(H^{2}\right) / 2$.

(1) If $\operatorname{gcd}(p r, q s)=1$, then we have $M_{X}^{H}\left(p^{2} r, p q H, q^{2} s\right) \cong M_{X}^{H}(r, H, s)$.

(2) Moreover if $\operatorname{rk} \operatorname{End}(X)=1$, then $M_{X}^{H}(r, H, s) \cong X$ if and only if $s=1$.

Proof. (1) First assume that $p q \neq 0$. Let $E$ be a semi-homogeneous vector bundle with $v(E)=$ $\left(p^{2} r, p q H, q^{2} s\right)$. Then we have $M_{X}^{H}\left(p^{2} r, p q H, q^{2} s\right) \cong X / K(E)$, where $K(E):=\left\{x \in X \mid T_{x}^{*}(E) \cong\right.$ $E\}$. By [M1, Cor. 7.8], $K(E)=p^{2} r K(p q H)$, where $K(H):=\left\{x \in X \mid T_{x}^{*}\left(\mathcal{O}_{X}(H)\right) \cong \mathcal{O}_{X}(H)\right\}$. We write $X=\mathbb{C}^{2} / \Lambda$, where $\Lambda$ is a lattice of rank 4 . Then there is a decomposition $\Lambda=$ $L_{1} \oplus L_{2}$ such that $L_{i} \cong \mathbb{Z}^{\oplus 2}(i=1,2)$ and $K(H)=\frac{1}{n} L_{1} / L_{1}=\frac{1}{r s} L_{1} / L_{1}$. Since $K(p q H)=$ $\frac{1}{p q r s} L_{1} / L_{1} \oplus \frac{1}{p q} L_{2} / L_{2}$, we have $p^{2} r K(p q H)=p\left(\frac{1}{q s} L_{1} / L_{1}\right) \oplus \operatorname{pr}\left(\frac{1}{q} L_{2} / L_{2}\right)$. Since $\operatorname{gcd}(p, q s)=1$ and $\operatorname{gcd}(p r, q)=1$, we have $p\left(\frac{1}{q s} L_{1} / L_{1}\right) \oplus \operatorname{pr}\left(\frac{1}{q} L_{2} / L_{2}\right)=\frac{1}{q s} L_{1} / L_{1} \oplus \frac{1}{q} L_{2} / L_{2}$. Thus $p^{2} r K(p q H)$ contains the subgroup $X_{q}$ of $q$-torsion points of $X$. Since the kernel of $q 1_{X}: X \rightarrow X$ is $X_{q}, q 1_{X}$ induces an isomorphism $p^{2} r K(p q H) / X_{q} \cong r K(H)$. Hence we have isomorphisms $M_{X}^{H}\left(p^{2} r, p q H, q^{2} s\right) \cong X /\left(p^{2} r K(p q H)\right) \cong X /(r K(H)) \cong M_{X}^{H}(r, H, s)$. 
Next we suppose $p q=0$. Then by the assumption we have $(p, q, r, s)=(1,0,1, n)$ or $(0,1, n, 1)$. For the first case, we have $M_{X}^{H}(1,0,0)=\widehat{X} \cong M_{X}^{H}(1, H, n)$. For the second case, $M_{X}^{H}(0,0,1)=$ $X \cong X /(n K(H)) \cong M_{X}^{H}(n, H, 1)$. Thus the conclusion holds.

(2) Assume that $\mathrm{rk} \operatorname{End}(X)=1$. If $X /(r K(H)) \cong X$, then we have a homomorphism $\psi$ : $X \rightarrow X /(r K(H)) \rightarrow X$. By our assumption, $m_{1} \psi=m_{2} 1_{X}$ for $m_{1} \in \mathbb{Z}_{>0}$ and $m_{2} \in \mathbb{Z}$. Then $r K(H)=\operatorname{ker}(\psi)=m_{1} X_{m_{2}}$. If $s \neq 1$, this is impossible. Hence $X /(r K(H)) \neq X$ if $s \neq 1$. If $s=1$, then clearly we have $X /(r K(H)) \cong X$.

We conclude this subsection with the next lemma, although it will not be used afterwards.

Lemma 8.7. Assume that $r, a \in \mathbb{Z}_{>0}$ satisfies $r a=n:=\left(H^{2}\right) / 2$. Then the dual variety of $M_{X}^{H}(r, H, a)$ is isomorphic to $M_{X}^{H}(a, H, r)$.

Proof. Set $Y:=M_{X}^{H}(r, H, a)$ and $Z:=M_{X}^{H}(a, H, r)$. We construct an isomorphism $Z \rightarrow \widehat{Y}$ using FMT. Since $\operatorname{gcd}(r, a)=1$, there exists a pair $(p, q) \in \mathbb{Z}^{2}$ such that $p r-q a=1$. Consider the moduli $\tilde{Y}:=M_{X}^{H}\left(p^{2} r, p q H, q^{2} a\right)$. Since $\left\langle\left(p^{2} r, p q H, q^{2} a\right),(a, H, r)\right\rangle=-(p r-q a)^{2}=-1$, there is a universal family $\mathbf{E}$ on $Z \times \widetilde{Y}$. We have $Y \cong \widetilde{Y}$ by (1). Thus there exits a universal family $\mathbf{F}$ on $Z \times Y$. Then choosing an appropriate integer $k$, we have a FMT $\Phi_{Z \rightarrow Y}^{\mathbf{F}[k]}$ which sends $\mathbb{C}_{z}(z \in Z)$ to a line bundle on $Y$. Retaking $\mathbf{F}$ as in Proposition 2.14 (4), we have $Z \cong M_{Y}^{\widehat{H}}(1,0,0)$.

8.3. Principally polarized case. In the case $n=1$, namely when $X$ is principally polarized, we have a simple description of the birational map.

First we note the next proposition.

Proposition 8.8. Assume that $X$ is an abelian surface with $\operatorname{NS}(X)=\mathbb{Z} H$ and $\left(H^{2}\right)=2$. Then $\operatorname{FM}(X)=\{X\}$.

Proof. If $\mathrm{NS}(X)=\mathbb{Z} H$, then a two-dimensional fine moduli of the stable sheaves on $X$ can be written as $M_{X}^{H}\left(p^{2} r, p q H, q^{2} a\right)$ with $p, q \in \mathbb{Z}$ and $r, a \in \mathbb{Z}_{>0}$ satisfying $\operatorname{gcd}(p r, q a)=1$ and $r a=$ $\left(H^{2}\right) / 2$. Then by Lemma 8.6 we have $\operatorname{FM}(X)=\left\{M_{X}^{H}(r, H, a) \mid \operatorname{gcd}(r, a)=1, r a=\left(H^{2}\right) / 2\right\} / \sim$. If moreover $\left(H^{2}\right)=2$, then $\operatorname{FM}(X)=\left\{M_{X}^{H}(1, H, 1)\right\}$. Since $M_{X}^{H}(1, H, 1) \cong \widehat{X} \cong X$, we have the consequence.

Next we recall the $G$ introduced in Definition 6.9, In the case $n=1, G$ coincides with $\mathrm{SL}(2, \mathbb{Z})$. Then the study of numerical solutions reduces to the theory of integral quadratic forms.

Definition 8.9. Two quadratic forms $f_{1}(x, y)=a x^{2}+2 b x y+c y^{2}$ and $f_{2}(x, y)=a^{\prime} x^{2}+2 b^{\prime} x y+c^{\prime} y^{2}$ defined over $\mathbb{Z}$ (that is, $a, b, c, a^{\prime}, b^{\prime}, c^{\prime} \in \mathbb{Z}$ ) are called equivalent if there exists a matrix $A \in$ $\mathrm{GL}(2, \mathbb{Z})$ such that ${ }^{t} A\left[\begin{array}{ll}a & b \\ b & c\end{array}\right] A=\left[\begin{array}{ll}a^{\prime} & b^{\prime} \\ b^{\prime} & c^{\prime}\end{array}\right]$. We denote it by $f_{1}(x, y) \equiv f_{2}(x, y)$.

The discriminant of the quadratic form $f(x, y)=a x^{2}+2 b x y+c y^{2}$ is defined as $\operatorname{det} f:=b^{2}-a c$.

The class number of quadratic forms of discriminant $D$ is the number of the equivalent classes of quadratic forms whose discriminant are $D$.

Theorem 8.10. Let $X$ be a principally polarized abelian surface with $\mathrm{NS}(X)=\mathbb{Z} H$. Let $v=(r, d H, a)$ be a Mukai vector satisfying the following condition.

$$
\ell:=\left\langle v^{2}\right\rangle / 2 \in \mathbb{Z}_{>0} \text { is not a square number, and }
$$

the class number of quadratic forms with discriminant $\ell$ is 1 .

Then the birational morphism $M_{X}^{H}(v) \cdots \rightarrow X \times \operatorname{Hilb}^{\ell} X$ is given by the following description.

(1) Take a Mukai vector $v_{1}=\left(p_{1}^{2}, p_{1} q_{1} H, q_{1}^{2}\right)$ which is of minimum rank among those satisfying $2 p_{1} q_{1} d-p_{1}^{2} a-q_{1}^{2} r=\epsilon$, where $\epsilon=1$ or -1 . 
(2) Then the matrix

$$
\gamma:= \pm\left[\begin{array}{cc}
q_{1} & \epsilon\left(d q_{1}-a p_{1}\right) \\
-p_{1} & \epsilon\left(d p_{1}-r q_{1}\right)
\end{array}\right] \in \operatorname{PSL}(2, \mathbb{Z})
$$

diagonalizes the matrix $Q_{v}=\left[\begin{array}{ll}r & d \\ d & a\end{array}\right]$, that is, ${ }^{t} \gamma Q_{v} \gamma=-\epsilon\left[\begin{array}{cc}1 & 0 \\ 0 & -\ell\end{array}\right]$.

(3) Set $v_{2}:=\left(p_{2}^{2}, p_{2} q_{2} H, q_{2}^{2}\right)$, where $q_{2}=-\epsilon\left(d q_{1}-a p_{1}\right)$ and $p_{2}=\epsilon\left(d p_{1}-r q_{1}\right)$. Then a general member of $M_{X}^{H}(v)$ has a semi-homogeneous presentation with numerical solution $v= \pm\left(\ell v_{1}-v_{2}\right)$. Moreover the FMT $\Phi:=\Phi_{X \rightarrow X}^{\mathbf{E}}$ such that $\theta(\Phi)=\gamma$ or the composition $\mathcal{D}_{X} \circ \Phi$ gives the birational correspondence $M_{X}^{H}(v) \cdots \rightarrow X \times \operatorname{Hilb}^{\ell}(X)$ up to shift.

Proof. The condition (8.3) implies that there exists at least one numerical solution for $v$. Then the conclusion follows from Proposition 8.2 .

Remark 8.11. It is conjectured that there are infinite number of real quadratic forms whose class number is one.

We close this paper with the comment on the birational types of lower dimensional moduli. By the above theorem, one is lead to the classification of quadratic forms $Q(x, y)=r x^{2}+2 d x y+a y^{2}$ of small discriminant $\ell=d^{2}-r a$. We search in the range $0<\ell<11$.

If $r=0$, then $Q=2 d x y+a y^{2}=2 d(x+\lambda y) y+(a-2 d \lambda) y^{2}$ for any $\lambda \in \mathbb{Z}$. Hence we assume $|a| \leq d$. If $a \neq 0$, then replacing $x$ and $y$ we reduce to the case $r>0$. If $r>0$, then we may assume that $0 \leq d \leq r / 2$. If $a \geq 0$, then from $0 \leq a=(d / r) d-\ell / r<d / 2 \leq r / 4$ we have $Q \equiv r^{\prime} x^{2}+2 d^{\prime} x y+a^{\prime} y^{2}$ with $0 \leq r^{\prime}<r$.

Thus by the action of $\mathrm{GL}(2, \mathbb{Z})$, we may assume

(i) $r=a=0$, or $\quad$ (ii) $r \neq 0$ and $0 \leq 2 d \leq r \leq-a$.

In the first case $r=a=0$, the quadratic form corresponding to a primitive Mukai vector is uniquely determined to be $Q=2 x y$.

In the second case, $a \leq 0$ yields $d \leq \sqrt{\ell}$. The above discussion allows us to assume $d \leq 3$. If $r=1$, then $d=0$ and we have $(r, d, \bar{a})=(1,0,-\ell)$. If $|a| \geq r>1$, then the possible values of $(r, d, a)$ are

$$
\begin{aligned}
& (2, d,-2) \quad d=0,1,2 ; \quad(2, d,-3) \quad d=0,1,2 ; \quad(2, d,-4) \quad d=0,1 ; \\
& (2, d,-5) \quad d=0 ; \quad(3, d,-3) \quad d=0,1 .
\end{aligned}
$$

We can remove the cases $(r, d, a)=(2,0,-2),(2,0,-4),(3,0,-3)$ since these correspond to nonprimitive Mukai vectors. Moreover we can find that $2 x^{2}-3 y^{2} \equiv 2 x^{2}-4 x y-y^{2} \equiv x^{2}-6 y$, $2 x^{2}+2 x y-3 y^{2} \equiv 2 x^{2}+6 x y+y^{2} \equiv x^{2}-7 y^{2}$ and $2 x^{2}-5 y^{2} \equiv 3 x^{2}+2 x y-3 y^{2}$.

We summarize the result in the table below.

\begin{tabular}{l|l||l|l}
$\ell$ & $Q_{v}$ & $\ell$ & $Q_{v}$ \\
\hline 1 & $2 x y, x^{2}-y^{2}$ & 6 & $x^{2}-6 y^{2}$ \\
2 & $x^{2}-2 y^{2}$ & 7 & $x^{2}-7 y^{2}$ \\
3 & $x^{2}-3 y^{2}$ & 8 & $x^{2}-8 y^{2}$ \\
4 & $x^{2}-4 y^{2}$ & 9 & $2 x^{2}+2 x y-4 y^{2}, x^{2}-9 y^{2}$ \\
5 & $2 x^{2}+2 x y-2 y^{2}, x^{2}-5 y^{2}$ & 10 & $3 x^{2}+2 x y-3 y^{2}, x^{2}-10 y^{2}$
\end{tabular}

Note that if $Q_{v}$ is not primitive and $\ell>1$, then $M_{X}^{H}(v)$ is not birationally equivalent to $X \times \operatorname{Hilb}^{\ell}(X)$. For example, if $r, a \in 2 \mathbb{Z}$, then $Q_{v} \not \equiv x^{2}-\ell y^{2}$.

The following proposition describes the birational classes of the moduli of dimension $\leq 20$.

Proposition 8.12. (1) If $\ell=1,2,3,4,6,7,8$, then $M_{X}^{H}(v)$ is birationally equivalent to $X \times$ $\operatorname{Hilb}^{\ell}(X)$.

(2) If $\ell=5$, then the birational type of $M_{X}^{H}(v)$ is either $M_{X}^{H}(2, H,-2)$ or $X \times \operatorname{Hilb}^{\ell}(X)$. 
(3) If $\ell=9$, then the birational type of $M_{X}^{H}(v)$ is either $M_{X}^{H}(2, H,-4)$ or $X \times \operatorname{Hilb}^{\ell}(X)$.

Proof. We only need to show that $M_{X}^{H}(2, H,-2), M_{X}^{H}(2, H,-4)$ and $X \times \operatorname{Hilb}^{\ell}(X)$ are birationally different. The case $M_{X}^{H}(2, H,-2)$ is shown in [Y1, Example 4.1]. The case $M_{X}^{H}(2, H,-4)$ can be shown similarly.

Remark 8.13. In the case $\ell=10$, we cannot distinguish $M_{X}^{H}(3, H,-3)$ and $X \times \operatorname{Hilb}^{10}(X)$ by the method of [Y1, Example 4.1].

\section{REFERENCES}

$[\mathrm{Hu}]$ D. Huybrechts, Fourier-Mukai transforms in algebraic geometry, Oxford University Press, 2006.

[HL] D. Huybrechts, M. Lehn, The geometry of moduli spaces of sheaves, Aspects of mathematics, Vieweg, 1997.

[M1] S. Mukai, Semi-homogeneous vector bundles on an abelian variety, J. Math. Kyoto Univ. 18 (1978), no. 2, 239-272.

[M2] S. Mukai, On Fourier functors and their applications to vector bundles on abelian surfaces (in Japanese), in the proceeding of "Daisuu-kikagaku Symposium" (Tôhoku University, June 1979), 76-93.

[M3] S. Mukai, On classification of vector bundles on abelian surfaces (in Japanese), RIMS Kôkyûroku 409 (1980), 103-127.

[M4] S. Mukai, Duality between $D(X)$ and $D(\widehat{X})$ with its application to Picard sheaves, Nagoya Math. J. 81 (1981), 153-175.

[M5] S. Mukai, Symplectic structure of the moduli space of sheaves on an abelian and K3 surface, Invent. Math. 77 (1984), 101-116.

[M6] S. Mukai, On the moduli space of bundles on $K 3$ surfaces. I, in Vector bundles on algebraic varieties, 341-413, Tata Inst. Fund. Res., 1987.

[M7] S. Mukai, Fourier Functor and its Application to the Moduli of Bundles on an Abelian Variety, Advanced Studies in Pure Mathematics, 10 (1987), 515-550, .

[M8] S. Mukai, Abelian variety and spin representation, preprint, 1998.

[Or] D. Orlov, Derived categories of coherent sheaves on abelian varieties and equivalences between them, Izv. Math. 66 (2002), no. 3, 569-594

[Y1] K. Yoshioka, Moduli spaces of stable sheaves on abelian surfaces, Math. Ann. 321 (2001), 817-884.

[Y2] K. Yoshioka, Twisted stability and Fourier-Mukai transforms I, Compositio Math. 138 (2003), 261-288.

[Y3] K. Yoshioka, Stability and the Fourier-Mukai transform II, Compositio Math. 145 (2009), 112-142.

[Y4] K. Yoshioka, Fourier-Mukai transform on abelian surfaces, to appear in Math. Ann., online version available at http://www.springerlink.com/content/100442/.

Shintarou Yanagida

Department of Mathematics, Faculty of Science, Kobe University, Kobe 657-8501, Japan

E-mail address: yanagida@math.kobe-u.ac.jp

Kōta Yoshioka

Department of Mathematics, Faculty of Science, Kobe University, Kobe 657-8501, Japan

MaX-Planck-Institut Für MATHEMATIK, Vivatsgasse 753111 BonN

E-mail address: yoshioka@math.kobe-u.ac.jp 